\title{
ESTABILIDADE DE EQUAÇÕES DIFERENCIAIS RETARDADAS COM ARGUMENTO SECCIONALMENTE CONTÍNUO
}

Suzinei Aparecida Siqueira Marconato

Orientador: Prof.Dr. Luiz Antonio Vieira de Carvalho

Tese apresentada ao Instituto de Ciências Matemáticas de São Carlos-USP, como parte dos requisitos para obtenção do título de Doutor em Ciências-Área: Matemática - Análise.

USP - São Carlos 1995 
Aos meus pais

Paulo e Águida 


\section{AGRADECIMENTOS}

Ao professor Luiz Antonio Vieira de Carvalho, pela confiança e orientação.

Aos professores José Geraldo dos Reis, Alexandre Nolasco de Carvalho e Maria Aparecida Bená, pela amizade e pelas valiosas discussões.

Ao meu marido José Carlos, pelo incentivo, pela compreensão e carinho que me dedicou durante todos estes anos.

À minha família, pelo carinho, pelo apoio e pela força interior que todos sempre me transmitiram.

Aos amigos do Departamento de Matemática da UNESP-Rio Claro, pela oportunidade concedida, pelo apoio e pelas valiosas discussões.

À Elizabeth, Laura e Sônia, funcionárias da área acadêmica do ICMSC-USP, pela eficiência e pelo carinho com que sempre me atenderam.

À CAPES, pelo auxílio concedido através do PICD.

À Maria Elisa, pela amizade e pelo capricho com que digitou este trabalho.

À todas as pessoas que, direta ou indiretamente, contribuíram para a realização deste trabalho. 


\section{ABSTRACT}

This work is concerned with the study of the stability of Retarded Differential Equations with Piecewise Continuous Argument (EPCA) and Discrete Equations, using Dichotomic Maps.

The definition of Dichotomic Map, and theorems of stability and asymptotic stability for the two cited equations, are established.

We show an important relationship between an EPCA and its associated discrete equation proving, under certain conditions, their equivalence in the study of stability.

An interesting aspect of the EPCA is that the stability of its null equilibrium with initial instant $n_{0} \in Z$, is equivalent to the stability with initial instant $\mathrm{t}_{\mathrm{o}} \in \mathfrak{R}$.

The developed methods are illustraded with applications through which we highlight the fact that the main advantage of those methods consist in the use of extremely simple functionals for the achievement of the desired results of stability. 


\section{RESUMO}

Este trabalho é sobre o estudo da estabilidade de Equações Diferenciais Retardadas com Argumento Seccionalmente Contínuo e de Equações Discretas, usando Funções Dicotômicas.

A definição de Função Dicotômica e teoremas de estabilidade e estabilidade assintótica para as duas equações citadas, são estabelecidos.

Evidenciamos a importante relação entre a equação diferencial e sua equação discreta associada provando, sob certas condições, a equivalência no estudo de estabilidade.

Um aspecto interessante da equação diferencial é que, a estabilidade do seu equilíbrio nulo com instante inicial $n_{0} \in Z$, é equivalente à sua estabilidade com instante inicial $\mathrm{t}_{\mathrm{o}} \in \mathfrak{R}$.

Os métodos apresentados são ilustrados com aplicações, onde observamos que a principal vantagem destes métodos consiste no uso de funcionais extremamente simples para a obtenção dos resultados desejados de estabilidade. 


\section{INDICE}

INTRODUÇÃO

CAPITULO 1 - Fatos Básicos e o Conceito de Função Dicotômica

1.1 - Equações Discretas ..................................................... 01

1.2 - Equações Diferenciais Funcionais Retardadas ........... 06

CAPITULO 2 - Resultados Gerais sobre a Equação Diferencial com Retardamento Seccionalmente Contínuo

2.1 - A Relação entre a Equação Diferencial (1.3) e sua Equação Discreta Associada .................................... 13

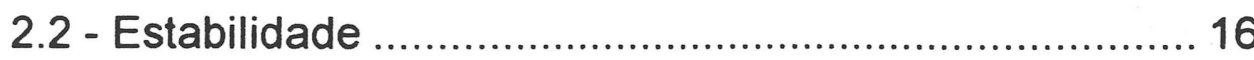

2.3 - Equação Autônoma nos Inteiros ................................. 20

CAPÍTULO 3 - Estudo da Estabilidade e Estabilidade Assintótica usando Funções Dicotômicas

3.1 - A Equação Discreta (1.1) ............................................. 23

3.2 - A Equação Diferencial (1.3) ......................................... 31

CAPÍTULO 4 - A Relação entre a Equação Diferencial e sua Equação Discreta Associada através de Funções Dicotômicas

4.1 - Caso Escalar da Equação Diferencial (2.5) ................. 43

4.2 - Caso Geral (Não Escalar) da Equação

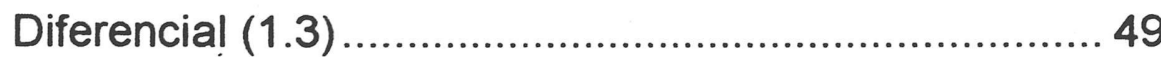

CAPÍTULO 5 - Aplicações da Teoria de Funções Dicotômicas 61

BIBLIOGRAFIA 87

APÊNDICE 90 


\section{INTRODUÇÃO}

Neste trabalho estudamos equações diferenciais năo autônomas do tipo

$$
\begin{aligned}
& x^{\prime}(t)=f(t, x(t), x([t])) \text { q.t.p., } t \geq t_{0} \\
& x_{t_{0}}=\varphi
\end{aligned}
$$

onde [ $\mathrm{t}$ ] denota "a parte inteira de $\mathrm{t}$ ". Elas podem ser consideradas como tipos especiais de Equações Diferenciais Funcionais, e são chamadas de Equações Diferenciais Retardadas com Argumento Seccionalmente Contínuo (EPCA).

Estas equações tem sido estudadas em alguns casos lineares e não lineares por Cooke e Wiener [8, 9, 10], Aftabizadeh e Wiener [1], Jayasree e Deo [15], Seifert [20] e outros. E equações do tipo neutro com este tipo de argumento, tem sido também discutidas por Cooke e Wiener [7].

A EPCA é um caso particular de

$$
x^{\prime}(t)=f(t, x(t), x(h(t))
$$

onde 0 argumento $h(t)$ tem intervalos de constância. Note que, no nosso caso, a funçăo $h(t)$ é descontínua. 
As equaçס̋es do tipo (A) passaram a ter a atençăo dos pesquisadores a partir de um artigo de A. D. Myshkis [18] onde o autor observou que năo existia uma teoria substancial para equações diferenciais com argumentos que são seccionalmente constantes ou seccionalmente contínuos.

Aplicações destas equações se apresentam na estabilização de sistemas de controle híbrido com retardamento na realimentação. Por um sistema híbrido, entendemos um sistema com uma instalação em processo contínuo com um controlador discreto (por amostragem).

No caso em que a função $f$ não depende diretamente da variável $t$, a EPCA tem estrutura de sistema dinâmico contínuo em intervalos de comprimento 1 , e portanto combina as propriedades de equações diferenciais e equações diferença.

A continuidade de uma solução num ponto de junção de quaisquer dois intervalos consecutivos, implica em relações recursivas para os valores da solução em tais pontos, originando a equação discreta associada.

Daí a importância do estudo da estabilidade de equaçōes discretas que também desenvolvemos neste trabalho, estendendo os resultados de Carvalho e Ferreira [5] sobre equações autônomas.

A teoria que apresentamos para a EPCA, é uma adaptação ao trabalho de Bená [2] que trabalha com Equações Diferenciais Funcionais Retardadas não Autônomas, utilizando o Método das Funções Dicotômicas, originalmente estabelecido por Carvalho e Ferreira [5] para equações discretas e posteriormente por Carvalho e Cooke [4] para equações diferenciais-diferença.

O Método de Funçōes Dicotômicas apresenta um avanço no estudo da estabilidade do equilíbrio de uma equação diferencial funcional (como também para equações discretas), dentro do contexto do Segundo Método 
de Liapunov e estende os Teoremas clássicos de Razumikhin, que permitem a determinaçăo de estabilidade em certos casos em que é muito complicada a construçăo de um funcional de Liapunov. O Método de Funçőes Dicotômicas apresenta vantagem na obtençăo de resultados de estabilidade, utilizando-se funções extremamente simples.

Este trabalho é composto de cinco capítulos. O primeiro restringe-se à apresentação de elementos básicos para o desenvolvimento do trabalho. No segundo capítulo, fazemos a relação entre a EPCA e sua equação discreta associada, observando características interessantes apresentadas pela equação discreta.

No capítulo 3, estabelecemos os teoremas que garantem estabilidade (estabilidade assintótica) para a EPCA e para equações discretas não autônomas, usando Funções Dicotômicas.

No capítulo 4, apresentamos uma comparação entre a aplicação do princípio de Dicotomia com relação à EPCA e à sua equação discreta associada.

No caso escalar, em que a função $f$ não depende diretamente da variável $t$, trabalhando com a função dicotômica $V(x)=x^{2} / 2$, obtemos equivalência de resultados para as duas equações.

No caso não escalar (geral), nem sempre podemos utilizar a mesma função dicotômica para as duas equações, porém, dada uma função $\mathrm{V}$ dicotômica (estritamente dicotômica) com relação à EPCA, definimos uma nova função a partir de $\mathrm{V}$, que terá a propriedade de dicotomia desejada para a equação discreta associada.

O capítulo 5 é destinado à aplicações do Método das Funções Dicotômicas. 


\section{Capitulo 1}

\section{FATOS BÁSICOS E O CONCEITO DE FUNÇÃO DICOTÓMICA}

Neste trabalho serão consideradas equações diferenciais funcionais com retardamento seccionalmente contínuo, que são tanto um tipo especial de equações diferenciais funcionais retardadas como também um caso particular de equações a diferenças finitas. Abordemos então, os fatos básicos sobre estas duas teorias.

\subsection{Equações Discretas}

Seja f: Ix $\mathfrak{R}^{\mathrm{n}} \rightarrow \mathfrak{R}^{\mathrm{n}}$ uma função contínua, $I=\left\{\mathrm{n}_{\mathrm{o}}, \mathrm{n}_{\mathrm{o}}+1, \cdots\right\}$, $f(n, 0)=0$ para todo $n \in I$ e consideremos a equação discreta:

$$
\begin{aligned}
& x_{n+1}=f\left(n, x_{n}\right), \quad n=n_{o}, n_{o}+1, \cdots \\
& x_{n_{o}}=x_{0}
\end{aligned}
$$

A solução de (1.1) é dada pela seqüência $x_{0}, f\left(n_{0}, x_{0}\right)$, $\mathrm{f}\left(\mathrm{n}_{\mathrm{o}}+1, \mathrm{f}\left(\mathrm{n}_{\mathrm{o}}, \mathrm{x}_{\mathrm{o}}\right)\right), \cdots$, que existe, é única e depende continuamente de $\mathrm{x}_{\mathrm{o}}$, simplesmente pelo fato de $f$ ser uma função contínua $[14,16]$.

Denotemos a solução por $\mathrm{x}\left(\mathrm{n}, \mathrm{n}_{\mathrm{o}}, \mathrm{x}_{\mathrm{o}}\right)$ (ou, quando não couber dúvida, $x_{n}\left(x_{0}\right)$ ). Como $f(n, 0)=0$ para todo $n \in I$, a solução através de $x_{0}=0$ é a solução nula, a qual é também chamada de "equilíbrio nulo".

Denotemos por $|\mathrm{x}|$, a norma Euclidiana de $\mathrm{x}$. 
A seguir, apresentamos algumas definiçסes e notaçס̄es, que serăo necessárias no decorrer deste trabalho.

Definiçăo 1.1: A soluçăo nula de (1.1) é estável (segundo Liapunov) se, dados $n_{0} \in N$ e $\varepsilon>0$, existir $\delta=\delta\left(\varepsilon, n_{0}\right)>0$ tal que, se $\left|x_{0}\right|<\delta$ entáo $\left|\mathrm{x}_{\mathrm{n}}\left(\mathrm{x}_{\mathrm{o}}\right)\right|<\varepsilon$, para todo $\mathrm{n} \geq \mathrm{n}_{\mathrm{o}}$.

Definição 1.2: A solução nula de (1.1) é assintoticamente estável se for estável e além disso existir $\gamma=\gamma\left(n_{0}\right)>0$ tal que se $\left|x_{0}\right|<\gamma$, então $\mathrm{x}_{\mathrm{n}}\left(\mathrm{x}_{\mathrm{o}}\right) \rightarrow 0$ quando $\mathrm{n} \rightarrow \infty$.

Definição 1.3: Seja $U \subset \mathfrak{R}^{\mathrm{n}}$, uma vizinhança da origem. Uma função contínua $\mathrm{V}: \mathrm{U} \rightarrow \Re$ é dita definida positiva se $\mathrm{V}(0)=0$ e $\mathrm{V}(\mathrm{x})>0$ para $\mathrm{x} \neq 0$ $\mathrm{x} \in \mathrm{U}$.

Definição 1.4: Uma função $\mathrm{V}: \mathfrak{R} \times \mathrm{U} \rightarrow \mathfrak{R}$ é dita definida positiva em $\mathrm{U}$, se $\mathrm{V}$ é contínua, $\mathrm{V}(\mathrm{t}, 0)=0$ para qualquer $\mathrm{t} \in \mathfrak{R}$ e existe uma funçăo $\alpha: \Re \rightarrow \Re$ contínua, estritamente crescente em $[0, \infty)$ com $\alpha(0)=0$, tal que $\mathrm{V}(\mathrm{t}, \mathrm{x}) \geq \alpha(|\mathrm{x}|) \quad$ para $(\mathrm{t}, \mathrm{x}) \in \mathfrak{R} \mathrm{xU}$.

Definição 1.5: Dada uma função $\mathrm{V}: \mathfrak{R} \times \mathfrak{R}^{\mathrm{n}} \rightarrow \mathfrak{R}$ e um número inteiro năo negativo $\mathrm{k}$, definimos a $\mathrm{k}$-ésima variaçăo de $\mathrm{V}$ ao longo das soluçöes de (1.1), por:

$\Delta_{\mathrm{k}} \mathrm{V}\left(\mathrm{n}, \mathrm{x}\left(\mathrm{n}, \mathrm{n}_{\mathrm{o}}, \mathrm{x}_{\mathrm{o}}\right)\right)=\mathrm{V}\left(\mathrm{n}, \mathrm{x}\left(\mathrm{n}, \mathrm{n}_{\mathrm{o}}, \mathrm{x}_{\mathrm{o}}\right)\right)-\mathrm{V}\left(\mathrm{n}-\mathrm{k}, \mathrm{x}\left(\mathrm{n}-\mathrm{k}, \mathrm{n}_{\mathrm{o}}, \mathrm{x}_{\mathrm{o}}\right)\right), \mathrm{n} \geq \mathrm{n}_{\mathrm{o}}+\mathrm{k}$

Dados: 
(i) uma funçăo continua $V$,

(ii) uma vizinhança $\Omega$ da origem em $\Re^{\mathrm{n}}$,

(iii) $n, j, k \in Z$ com $k \geq 2, n \geq n_{0}+k$ e $j=1,2, \cdots, k$,

(iv) $\mathrm{p}: \mathfrak{R}_{+} \rightarrow \mathfrak{R}_{+}$, uma funçăo contínua, năo decrescente, $\mathrm{p}(\mathrm{y})>\mathrm{y}$, $y \in(0, \delta)$ para algum $\delta>0$ fixado,

passamos a definir os seguintes subconjuntos do $\mathfrak{R}^{\mathrm{n}}$ :

$$
\begin{aligned}
& \Omega_{+}^{j}(n, k)=\left\{y \in \Omega: \Delta_{j} V(n, x(n, n-k, y))>0\right\}, \\
& \Omega_{-}^{j}(n, k)=\left\{y \in \Omega: \Delta_{j} V(n, x(n, n-k, y))<0\right\}, \\
& \Omega_{0}^{j}(n, k)=\left\{y \in \Omega: \Delta_{j} V(n, x(n, n-k, y))=0\right\} e \\
& \Omega_{p-}^{j}(n, k)=\{y \in \Omega: p(V(n, x(n, n-k, y)))-V(n-j, x(n-j, n-k, y))<0\} .
\end{aligned}
$$

Dado um conjunto $A, A^{*}$ representa o conjunto $A \backslash\{0\}, \bar{A}$, o fecho de A e $\Omega_{0-}^{\mathrm{j}}(\mathrm{n}, \mathrm{k})=\Omega_{-}^{\mathrm{j}}(\mathrm{n}, \mathrm{k}) \cup \Omega_{\mathrm{o}}^{\mathrm{j}}(\mathrm{n}, \mathrm{k})$.

Definição 1.6: Dadas uma vizinhança $\Omega$ da origem em $\Re^{\mathrm{n}}$ e uma função contínua $\mathrm{V}: \mathfrak{R} \times \mathfrak{R}^{\mathrm{n}} \rightarrow \mathfrak{R}$, dizemos que $\mathrm{V}$ é dicotómica com relaçăo à equaçăo (1.1) (em $\Omega$ ) se, qualquer que seja $n_{0} \in Z$, pudermos encontrar $N=N\left(n_{0}\right), N \in Z_{+}, N \geq 2$, tal que para todo inteiro $n \geq n_{0}+N$, existe em correspondência um outro inteiro $k=k\left(n_{0}, n\right), 2 \leq k \leq n-n_{0}$, para o qual se verifica:

$$
\left[\bar{\Omega}_{+}^{1}(\mathrm{n}, \mathrm{k}) \cup \Omega_{\mathrm{o}}^{1}(\mathrm{n}, \mathrm{k})\right] \subset \bigcup_{j=2}^{\mathrm{k}} \Omega_{\mathrm{o}-}^{\mathrm{j}}(\mathrm{n}, \mathrm{k})
$$


Definiçăo 1.7: Se V for dicotómica, e além disso existirem um inteiro $M, 2 \leq M<\infty$ e uma funçăo $p$ satisfazendo (iv) tais que:

(i) $k=k\left(n_{0}, n\right) \leq M$, para todo $n \geq n_{o}+N$,

(ii) $\left[\bar{\Omega}_{+}^{1}(\mathrm{n}, \mathrm{k}) \cup \Omega_{\mathrm{o}}^{1}(\mathrm{n}, \mathrm{k})\right]^{*} \subset \bigcup_{j=2}^{\mathrm{k}} \Omega_{\mathrm{p}-(\mathrm{n}, \mathrm{k}) \mathrm{e}}^{\mathrm{j}}$

(iii) sempre que $x_{n-k}\left(x_{o}\right) \rightarrow \bigcap_{j=1}^{k} \Omega_{0}^{j}(n, k)$, então necessariamente $\mathrm{x}_{\mathrm{n}}\left(\mathrm{x}_{\mathrm{o}}\right) \rightarrow 0$ quando $\mathrm{n} \rightarrow \infty$,

dizemos que $\mathrm{V}$ é estritamente dicotómica (em $\Omega$ ) com relação à equação (1.1).

Coloquialmente, a Definição (1.6) nos diz que V é dicotômica com relação à equação (1.1) em $\Omega$, se para todo $\mathrm{n}$ e todo $\mathrm{x} \in \Omega$ onde a primeira variação de $\mathrm{V}$ ao longo da solução é não negativa, existe um instante $(\mathrm{n}-\mathrm{j})$ anterior à $(n-1)$, tal que a j-ésima variaçăo de $\mathrm{V}$ é não positiva. Ou seja, apesar do crescimento de $\mathrm{V}$ ao longo da solução no ponto $\mathrm{n}$, temos um decrescimento de $\mathrm{V}$ se comparado com um certo ponto $\overline{\mathrm{n}}$ anterior à $(\mathrm{n}-1)$.

E no caso da Definição (1.7), V é estritamente dicotômica com relação à equação (1.1) em $\Omega$, se ocorrer a situação anterior para $x \neq 0$, com a j-ésima variação de $V$, estritamente negativa. $E$ mais, a solução tal que a função $\mathrm{V}$ ao longo da solução tende a se tornar constante, necessariamente tal solução tende a zero, quando $n \rightarrow \infty$.

Salientamos que, na definição de dicotomia para o caso autônomo dada em [3], o valor $\mathrm{k}$ é constante e na Definiçăo (1.6) é permitida a 
variaçăo de $k$ com relaçăo à variável $n$, e em particular, até mesmo o caso em que $k(n) \rightarrow \infty$ quando $n \rightarrow \infty$.

E mais, o processo de dicotomia năo necessariamente se inicia no instante $n_{0}$ e sim, a partir de $n_{0}+N$, para algum inteiro năo negativo $N=N\left(n_{0}\right)$.

Com relação à dicotomia estrita, na Definiçăo (1.7) exige-se a existência de uma funçăo $p$ com certas propriedades específicas, fundamentais na prova de estabilidade assintótica.

No caso autônomo, como é natural, as condições exigidas são menos restritivas, não havendo necessidade da existência da função $\mathrm{p}$, conforme se constata em [3].

Para a equação autônoma

$$
x_{n+1}=f\left(x_{n}\right), n \in N
$$

com condição inicial $x_{0}=y$ e que apresenta uma função dicotômica $V$ com constante k finita, as Definiçōes (1.6) e (1.7) podem ser dadas por:

Definição 1.6-A: Dadas uma vizinhança $\Omega$ da origem em $\mathfrak{R}^{\mathrm{n}}$ e uma função contínua $\mathrm{V}: \Omega \rightarrow \Re$, dizemos que $\mathrm{V}$ é dicotômica com relaçăo à equação (1.1-A) (em $\Omega$ ) se existir um inteiro $k$ finito, $k \geq 2$, tal que:

$$
\left[\bar{\Omega}_{+}^{1}(k) \cup \Omega_{0}^{1}(k)\right] \subset \bigcup_{j=2}^{k} \Omega_{0-}^{j}(k) .
$$

Definição 1.7-A: Se V for dicotômica e além disso: 
(i) $\left[\bar{\Omega}_{+}^{1}(k) \cup \Omega_{0}^{1}(k)\right]^{\bullet} \subset \bigcup_{j=2}^{k} \Omega_{-}^{j}(k)$

(ii) $\Omega_{\mathrm{o}}^{1}(\mathrm{k}) \cap \Omega_{\mathrm{o}}^{\mathrm{k}}(\mathrm{k})=\{0\}$,

dizemos então que $\mathrm{V}$ é estritamente dicotômica com relaçăo à equaçăo (1.1-A) (em $\Omega$ ).

Neste caso, estamos usando as seguintes definições equivalentes:

$$
\begin{aligned}
& \Omega_{+}^{\mathrm{j}}(\mathrm{k})=\left\{\mathrm{y} \in \Omega: \Delta_{\mathrm{j}} \mathrm{V}\left(\mathrm{x}_{\mathrm{k}}(\mathrm{y})\right)>0\right\}, \\
& \Omega_{-}^{\mathrm{j}}(\mathrm{k})=\left\{\mathrm{y} \in \Omega: \Delta_{\mathrm{j}} \mathrm{V}\left(\mathrm{x}_{\mathrm{k}}(\mathrm{y})\right)<0\right\}, \\
& \Omega_{\mathrm{o}}^{\mathrm{j}}(\mathrm{k})=\left\{\mathrm{y} \in \Omega: \Delta_{\mathrm{j}} \mathrm{V}\left(\mathrm{x}_{\mathrm{k}}(\mathrm{y})\right)=0\right\} \mathrm{e} \\
& \Omega_{0-}^{\mathrm{j}}(\mathrm{k})=\Omega_{-}^{\mathrm{j}}(\mathrm{k}) \cup \Omega_{\mathrm{o}}^{\mathrm{j}}(\mathrm{k}), \mathrm{j}=1,2, \cdots, \mathrm{k} .
\end{aligned}
$$

\subsection{Equações Diferenciais Funcionais Retardadas}

Consideremos a equação diferencial funcional retardada não autônoma:

$$
\begin{aligned}
& x^{\prime}(t)=g\left(t, x_{t}\right), \quad t \geq t o \\
& x_{t_{o}}=\varphi
\end{aligned}
$$


onde $g: \mathfrak{R} \times \mathrm{C} \rightarrow \mathfrak{R}^{n}$ é uma funçăo continua que satisfaz condiçóes suficientes para garantir a unicidade e dependéncia contínua das soluçóes de (1.2) em relaçăo às condiçóes iniciais.

E $g(t, 0)=0, \varphi \in C=C\left([-r, 0], \Re^{n}\right), r>0$; onde $C$ indica o espaço de Banach das aplicaçóes continuas $\varphi$ de $[-r, 0]$ em $\Re^{n}$, com a norma:

$$
\|\varphi\|=\sup \{|\varphi(\theta)|: \theta \in[-r, 0]\} .
$$

Dada uma funçăo contínua $\mathrm{x}:\left[\mathrm{t}_{\mathrm{o}}-\mathrm{r}, \mathrm{t}_{\mathrm{o}}+\zeta\right) \rightarrow \mathfrak{R}^{\mathrm{n}}, \zeta>0$, definimos para $t \in\left[t_{0}, t_{0}+\zeta\right)$, a função $x_{t}:[-r, 0] \rightarrow \Re^{n}$ da seguinte forma: $x_{t}(\theta)=x(t+\theta), \theta \in[-r, 0]$ onde $x_{t} \in C$.

Usaremos a notação $x\left(\cdot, t_{0}, \varphi\right)$ para a solução de (1.2), onde $x_{t_{\mathrm{o}}}\left(\cdot, t_{0}, \varphi\right)=\varphi$ e $x_{t}\left(\cdot, t_{o}, \varphi\right)(\theta)=x\left(t+\theta, t_{o}, \varphi\right), \theta \in[-r, 0]$.

A soluçăo através de $\varphi=0$ é a soluçăo nula, a qual é também chamada de "equilibrio nulo".

Mesmo no caso em que a função g em (1.2) não é contínua, podemos estender os resultados de existência, unicidade e dependência contínua no sentido de Carathéodory, de acordo com a seguinte definiçăo:

Definição 1.8: Uma funçăo $g: \Omega \subset \Re \times C \rightarrow \Re^{\mathrm{n}} \quad(\Omega$ : subconjunto aberto de $\Re \times C$ ) satisfaz condiçăo de Carathéodory sobre $\Omega$, se $g(t, \varphi)$ é mensurável em $t$ para cada $\varphi$ fixa; contínua em $\varphi$ para cada t fixo, e para todo par fixo $(t, \varphi) \in \Omega$, existe uma vizinhança $U(t, \varphi)$ e uma função $m$ Lebesgue integrável, tal que

$$
|\mathrm{g}(\mathrm{s}, \psi)| \leq \mathrm{m}(\mathrm{s}), \quad(\mathrm{s}, \psi) \in \mathrm{U}(\mathrm{t}, \varphi)
$$


Por exemplo, se g é contínua, é fácil constatar que $g$ satisfaz condiçăo de Carathéodory [13].

Com a hipótese de $\mathrm{g}$ satisfazer a condiçăo de Carathéodory, a soluçăo $x\left(\cdot, t_{0}, \varphi\right)$ de (1.2) definida em $\left[t_{0}-r, t_{0}+A\right]$ para algum $A>0$, é absolutamente continua em $\left[t_{0}, t_{0}+A\right]$ e satisfaz $x^{\prime}(t)=g\left(t, x_{t}\right)$ em quase toda parte de $\left[t_{0}, t_{0}+A\right]$ (q.t.p.).

A teoria geral de Equações Diferenciais Funcionais Retardadas, pode ser encontrada em $[11,13]$.

Vejamos agora a equação diferencial funcional com retardamento seccionalmente contínuo, para a qual todo este trabalho está voltado.

Seja a equação

$$
\begin{aligned}
& x^{\prime}(t)=f(t, x(t), x([t])) \text { q.t.p., } \quad t \geq t_{0} \\
& x_{t_{0}}=\varphi
\end{aligned}
$$

onde [t] denota "a parte inteira de $\mathrm{t}$ ", $\varphi \in \mathrm{C}\left([-1,0], \mathfrak{R}^{\mathrm{n}}\right), \mathrm{f}: \mathfrak{R} \times \mathfrak{R}^{2 \mathrm{n}} \rightarrow \mathfrak{R}^{\mathrm{n}}$ é uma função contínua com $f(t, 0,0)=0$.

Vamos considerar que $f$ satisfaz condições suficientes para garantir unicidade e dependência contínua das soluções de (1.3) em relação às condições iniciais.

O retardamento é seccionalmente contínuo pois $r(t)=t-[t]$ é uma função contínua em todo intervalo $[n, n+1), n \in Z$ e descontínua nos valores inteiros.

A equação (1.3) é equivalente à equação (1.2) com

$$
g(t, \varphi)=f(t, \varphi(0), \varphi([t]-t)), \quad t \geq t_{0}
$$


Observemos que $\mathrm{g}$ é descontínua em $\mathrm{t}$, devido à descontinuidade do retardamento $r(t)$. Mas como $f$ é contínua, obtemos o seguinte resultado sobre $g$ :

Lema 1.1: Se $f$ é contínua em $\mathfrak{R} \times \mathfrak{R}^{2 n}$, entăo a funçăo $\mathrm{g}$ dada por (1.4), satisfaz condiçăo de Carathéodory sobre $\mathfrak{R}$ x C.

Prova-se, sem dificuldades, este resultado trabalhando-se com a norma da soma em $\mathfrak{R}$ x $C$, a norma do supremo em $C$, e com a norma do máximo em $\mathfrak{R} \times \mathfrak{R}^{2 \mathrm{n}}$ (veja Apêndice).

Assim, a equação (1.3) é um caso particular da equação (1.2), com a ressalva de que $\mathrm{g}$ não é contínua, mas satisfaz a condição de Carathéodory sobre $\mathfrak{R} \times \mathbf{C}$.

Em se tratando da equaçăo (1.3), devemos adotar o seguinte conceito de soluçăo (veja Cooke e Wiener [7]):

Definição 1.9: Uma solução de (1.3) em $\left[t_{0}, b\right)$ é uma função $x(t)$ que satisfaz as condições:

(i) $x(t)$ é contínua em $\left[t_{0}, b\right)$,

(ii) a derivada $x^{\prime}(t)$ existe em cada ponto $t \in\left[t_{0}, b\right)$, com a possivel exceção dos pontos $[t] \in\left[t_{0}, b\right)$, onde derivadas laterais existem,

(iii) equaçăo (1.3) está satisfeita em cada intervalo $[n, n+1) \subset\left[t_{0}, b\right)$, $\mathrm{n} \in \mathrm{Z}$. 
Vejamos agora mais algumas definiçőes e notaçőes que serăo necessárias.

Definiçăo 1.10: A soluçăo nula de (1.3) é dita estável (no sentido de Liapunov) se dados $\mathrm{t}_{\mathrm{o}} \in \mathfrak{R}$ e $\varepsilon>0$, existir $\delta=\delta\left(\varepsilon, \mathrm{t}_{\mathrm{o}}\right)>0$ tal que se $\|\varphi\|<\delta$ entăo $\left\|\mathrm{x}_{\mathrm{t}}\left(\cdot, \mathrm{t}_{\mathrm{o}}, \varphi\right)\right\|<\varepsilon$ para $\mathrm{t} \geq \mathrm{t}_{\mathrm{o}}$.

Definição 1.11: A soluçăo nula de (1.3) é dita assintoticamente estável se for estável e além disso, existir $\gamma=\gamma\left(t_{0}\right)>0$ tal que se $\|\varphi\|<\gamma$, então $x\left(t, t_{0}, \varphi\right) \rightarrow 0$ quando $t \rightarrow \infty$.

Definição 1.12: Seja $V: \Re \times \Re^{\mathbb{n}} \rightarrow \Re$ uma função contínua. Definimos a "derivada de $\mathrm{V}$ ao longo das soluçóes de (1.3), $\mathrm{V}^{\prime}(\mathrm{t}, \varphi)$ ", por

$$
\mathrm{V}^{\prime}(\mathrm{t}, \varphi)=\lim _{\mathrm{h} \rightarrow 0^{+}} \sup \frac{1}{\mathrm{~h}}[\mathrm{~V}(\mathrm{t}+\mathrm{h}, \mathrm{x}(\mathrm{t}+\mathrm{h}, \mathrm{t}, \varphi))-\mathrm{V}(\mathrm{t}, \mathrm{x}(\mathrm{t}, \mathrm{t}, \varphi))]
$$

Dados:

(i) uma função diferenciável $\mathrm{V}$,

(ii) uma vizinhança $\Omega$ da origem em $C$,

(iii) $\mathrm{t}, \mathrm{T} \in \mathfrak{R}$ com $\mathrm{T}>0, \mathrm{t} \geq \mathrm{t}_{\mathrm{o}}+\mathrm{T}$,

(iv) $\mathrm{p}: \mathfrak{R}_{+} \rightarrow \mathfrak{R}_{+}$uma função contínua, não decrescente, $\mathrm{p}(\mathrm{y})>\mathrm{y}$ $y \in(0, \delta)$, para algum $\delta>0$ fixado,

definimos os seguintes subconjuntos de $\mathrm{C}$ : 


$$
\begin{aligned}
\Omega_{+}^{\prime}(t, T)= & \left\{\psi \in \Omega: V^{\prime}(t, x,(\cdot t-T, \psi))>0\right\}, \\
\Omega_{0}^{\prime}(t, T)= & \left\{\psi \in \Omega: V^{\prime}\left(t, x_{t}(\cdot, t-T, \psi)\right)=0\right\}, \\
\Omega_{-}^{s}(t, T)= & \{\psi \in \Omega: V(t, x(t, t-T, \psi))-V(t-s, x(t-s, t-T, \psi))<0\}, \\
& s \in(0, T], \\
\Omega_{0}^{s}(t, T)= & \{\psi \in \Omega: V(t, x(t, t-T, \psi))-V(t-s, x(t-s, t-T, \psi))=0\}, \\
& s \in(0, T], \\
\Omega_{p}^{s}(t, T)= & \{\psi \in \Omega: p(V(t, x(t, t-T, \psi))-V(t-s, x(t-s, t-T, \psi))<0\}, \\
& s \in(0, T], \\
\Omega_{0-}^{s}(t, T)= & \Omega_{-}^{s}(t, T) \cup \Omega \Omega_{0}^{s}(t, T) . \\
& s \in(0, T], \\
\Omega_{p_{0}}^{s}(t, T)= & \{\psi \in \Omega: p(V(t, x(t, t-T, \psi))-V(t-s, x(t-s, t-T, \psi)) \leq 0\}, \\
&
\end{aligned}
$$

Definição 1.13: Dadas uma vizinhança $\Omega$ da origem em $C$ e uma função diferenciável $\mathrm{V}: \mathfrak{R} \times \mathfrak{R}^{\mathrm{n}} \rightarrow \mathfrak{R}$, dizemos que $\mathrm{V}$ é dicotómica com relaçăo à equaçăo (1.3) (em $\Omega$ ) se, qualquer que seja $t_{0} \in \mathfrak{R}$, pudermos encontrar $\mu=\mu\left(t_{0}\right)>0$, tal que para todo $t \geq t_{0}+\mu$ existe em correspondência um $\mathrm{T}=\mathrm{T}\left(\mathrm{t}_{\mathrm{o}}, \mathrm{t}\right), 0<\mathrm{T} \leq \mathrm{t}-\mathrm{t}_{\mathrm{o}}$ para o qual se verifica:

$$
\left[\bar{\Omega}_{+}^{\prime}(t, T) \cup \Omega_{0}^{\prime}(t, T)\right] \subset \bigcup_{s} \Omega_{o-}^{s}(t, T), s \in(0, T]
$$


Definiçăo 1.14: Se $V$ for dicotómica, e além disso existir um $M$, $0<\mathrm{M}<\infty$ e uma funçăo $\mathrm{p}$ satisfazendo (iv) tais que:

(i) $\mathrm{T}=\mathrm{T}\left(\mathrm{t}_{\mathrm{o}}, \mathrm{t}\right) \leq \mathrm{M}$ para todo $\mathrm{t} \geq \mathrm{t}_{\mathrm{o}}+\mu$,

(ii) $\left[\bar{\Omega}_{+}^{\prime}(t, T) \cup \Omega_{0}^{\prime}(t, T)\right]^{*} \subset \bigcup_{s} \Omega_{p}^{s}(t, T), s \in(0, T] \quad e$

(iii) sempre que $x_{t-T} \rightarrow \bigcap_{s}\left[\Omega_{0}^{\prime}(t, T) \cap \Omega_{o}^{s}(t, T)\right], s \in(0, T]$, entăo necessariamente $\mathrm{x}_{\mathrm{t}} \rightarrow 0$ quando $\mathrm{t} \rightarrow \infty$,

dizemos que $\mathrm{V}$ é estritamente dicotómica com relação à equação (1.3).

Coloquialmente, a Definição (1.13) nos diz que $\mathrm{V}$ é dicotômica com relação à equação (1.3) em $\Omega$, se para todo t e toda $\psi \in \Omega$ onde a derivada de $\mathrm{V}$ ao longo da solução é não negativa, existe um instante $\overline{\mathrm{t}}$ anterior à $\mathrm{t}$, tal que a variaçăo de $\mathrm{V}$ entre os instantes $\mathrm{t}$ e $\overline{\mathrm{t}}$ é não positiva.

E no caso da Definiçăo (1.14), V é estritamente dicotômica com relação à equação (1.3) em $\Omega$, se ocorrer a situaçăo anterior para $\psi \neq 0$, com a variaçăo de $\mathrm{V}$ entre os instantes $\mathrm{t}$ e $\overline{\mathrm{t}}$, estritamente negativa, envolvendo uma função $p$ com certas propriedades específicas.

$E$ mais, se a derivada de $\mathrm{V}$ com relaçăo à $\mathrm{t}$ ao longo de uma soluçăo, tende a zero quando $t \rightarrow \infty$ e tende a se tornar uma funçăo constante em intervalos de amplitude $T$, então necessariamente esta soluçăo tende à soluçăo nula quando $\mathrm{t} \rightarrow \infty$. 


\section{Capitulo 2}

\section{RESULTADOS GERAIS SOBRE A EQUAÇĀO DIFERENCIAL COM RETARDAMENTO SECCIONALMENTE CONTÍNUO}

\subsection{A Relação entre a Equação Diferencial (1.3) e sua Equação Discreta Associada}

Sejam $x(t)$ solução de (1.3) com intervalo maximal de existência $\left[t_{0}, b\right), e n_{o}$ o primeiro inteiro maior ou igual à $t_{0}$.

Para $n \in Z$, seja $x_{n}(t)$ a restrição da solução $x(t)$ no intervalo

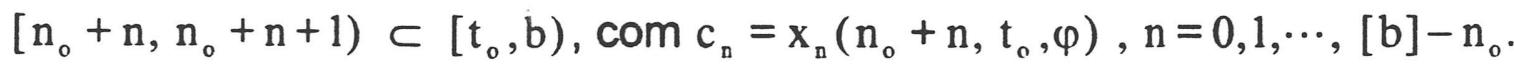

Cada restrição $\mathrm{x}_{\mathrm{n}}(\mathrm{t})$ satisfaz a equação diferencial ordinária:

$$
\begin{aligned}
& x^{\prime}(t)=f\left(t, x(t), c_{n}\right) \\
& x\left(n_{0}+n\right)=c_{n}
\end{aligned}
$$

para $\mathrm{t} \in\left[\mathrm{n}_{\mathrm{o}}+\mathrm{n}, \mathrm{n}_{\mathrm{o}}+\mathrm{n}+1\right), \mathrm{n}=0,1, \cdots,[\mathrm{b}]-\mathrm{n}_{\mathrm{o}}$.

Devido à continuidade da solução nos valores inteiros, devemos ter

$$
c_{n+1}=x_{n}\left(n_{o}+n+1\right), \quad n=0,1, \cdots,[b]-n_{0} .
$$

Assim, temos a equação discreta associada à equação (1.3), dada por 


$$
c_{n+1}=c_{n}+\int_{n_{0}+n}^{n_{0}+n+1} f\left(s, x(s), c_{n}\right) d s
$$

que pode ser vista como

$$
c_{n+1}=h\left(n, c_{n}\right), \quad n=0,1, \cdots,[b]-n_{0},
$$

onde $\mathrm{h}: \mathrm{I}$ x $\mathfrak{R}^{\mathrm{n}} \rightarrow \mathfrak{R}^{\mathrm{n}}$ é uma função contínua com $\mathrm{I}=\left\{0,1, \cdots,[\mathrm{b}]-\mathrm{n}_{\mathrm{o}}\right\}$.

Observemos que o estudo da equação discreta associada à equação (1.3) é importante devido à sua íntima ligação com esta equação: elas coincidem nos instantes inteiros.

Note que, em geral, isto não ocorre nas discretizações de uma equação diferencial ordinária.

Como ilustração, consideremos as equações

$$
\begin{aligned}
& x^{\prime}(t)=-x(t)+x^{3}([t])-\mu x([t]) \quad q \cdot t \cdot p ., \quad t \geq 0 \\
& x^{\prime}(t)=-(1+\mu) x(t)+x^{3}(t), \quad t \geq 0
\end{aligned}
$$

com condição inicial $\mathrm{x}(0)=\mathrm{c}_{\mathrm{o}}, \mathrm{c}_{\mathrm{o}} \in \mathfrak{R}$ e $\mu>0$.

Inicialmente, calculemos a equação discreta associada à equação (2.2).

No intervalo $[n, n+1), n \in N$, com a condição $x(n)=c_{n}$, a equação (2.2) reduz-se à:

$$
x^{\prime}(t)=-x(t)+c_{n}^{3}-\mu c_{n}
$$


que tem como soluçăa:

$$
x_{n}(t)=c_{n}\left(c_{n}^{2}-\mu\right)+c_{n}\left[1-\left(c_{n}^{2}-\mu\right)\right] \exp (n-t), \quad t \in[n, n+1) .
$$

Devido à continuidade da soluçăo $x(t)$ nos pontos $t=n, n \in N$, devemos ter:

$$
c_{n}=x_{n-1}(n)=c_{n-1}\left[\left(c_{n-1}^{2}-\mu\right)\left(1-e^{-1}\right)+e^{-1}\right], \quad n \in N .
$$

Para o caso $c_{0}=1 / 2$ e $\mu=1$, temos o seguinte esboço da solução:

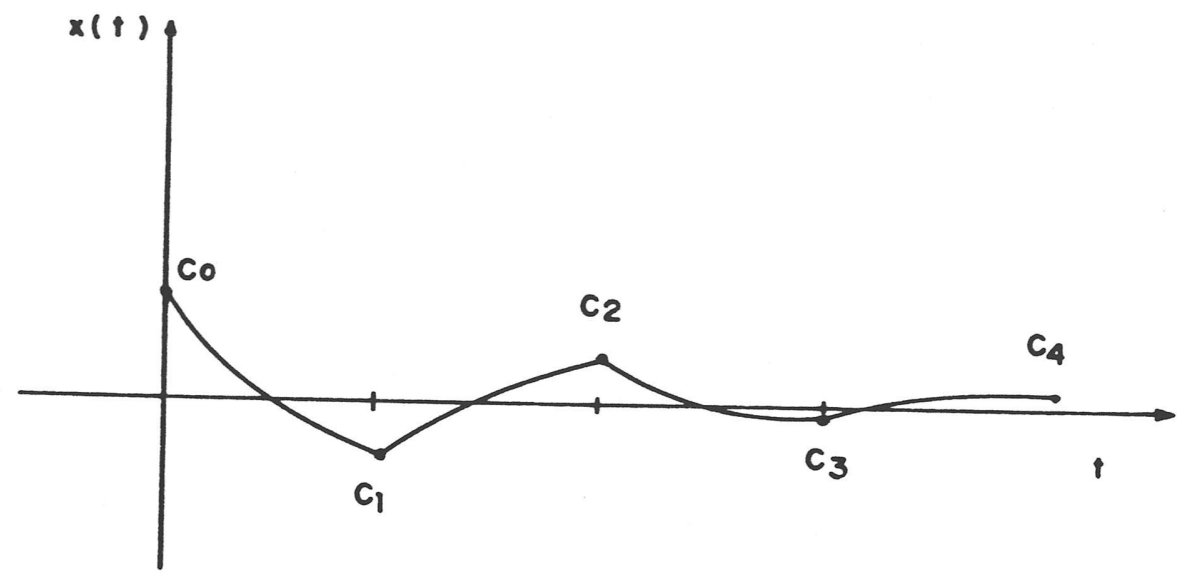

Para $c_{0}=1 / 2$ e $\mu=1$, a solução da equação (2.3) é dada por:

$$
x(t)=\sqrt{2}(1+7 \exp (4 t))^{-1 / 2}, \quad t \geq 0 .
$$

Usando, por exemplo, a técnica de aproximaçăo de $x^{\prime}(t)$ por $[x(t+h)-x(t)] / h$ ( Método de Euler), temos o seguinte: Para $h>0$ fixo $e$ $\mathrm{t}=\mathrm{nh}, \mathrm{n} \in \mathrm{N}, \mathrm{x}((\mathrm{n}+1) \mathrm{h})-\mathrm{x}(\mathrm{nh})=\mathrm{hf}(\mathrm{x}(\mathrm{nh}))$, e para $\mathrm{x}(\mathrm{nh})=\mathrm{x}_{\mathrm{n}}$, temos que $x_{n+1}=x_{n}+h f\left(x_{n}\right)=F\left(x_{n}\right)$. 
Para a equaçăo diferencial (2.3), temos como equaçăo aproximante, $x_{n+1}=x_{n}\left[1+h\left(x_{n}^{2}-1-\mu\right)\right]$, onde estes valores năo necessariamente coincidem com os valores da soluçăo de (2.3).

\subsection{Estabilidade:}

Se o instante inicial para a equação (1.3) for um número inteiro $n_{0}$, torna-se desnecessário trabalhar no espaço $\mathrm{C}$, bastando analisar o seguinte problema equivalente:

$$
\begin{aligned}
& x^{\prime}(t)=f(t, x(t), x([t])) \quad \text { q.t.p., } \quad t \geq n_{o} \\
& x\left(n_{o}\right)=c_{0}
\end{aligned}
$$

$\operatorname{com} c_{o} \in \Re^{n}$.

Um aspecto interessante da equação (1.3) é que a estabilidade do seu equilíbrio nulo com instante inicial $n_{0} \in Z$, é equivalente à sua estabilidade com instante inicial $t_{0} \in \mathfrak{R}$, como se segue:

Definição 2.1: O equilíbrio nulo de (1.3) é estável nos inteiros, se a solução de (2.4) é tal que:

dados $n_{0} \in Z$ e $\varepsilon>0$, existe um $\delta=\delta\left(\varepsilon, n_{0}\right)>0$, tal que se $\left|c_{0}\right|<\delta$ então $\left|\mathrm{x}\left(\mathrm{t}, \mathrm{n}_{\mathrm{o}}, \mathrm{c}_{\mathrm{o}}\right)\right|<\varepsilon$, para todo $\mathrm{t} \geq \mathrm{n}_{\mathrm{o}}$.

Fazemos a seguir a relação entre esta definição e a Definição geral (1.10) de estabilidade, dada no capítulo 1. 
Lema 2.1: As Definiçర̋es (1.10) e (2.1) săo equivalentes.

Demonstraçăo: É claro que a Definiçăo (1.10) implica na Definiçăo (2.1). Provemos a recíproca. Supondo válida a Definiçăo (2.1), dados $t_{0} \in \mathfrak{R}$ e $\varepsilon>0$, provemos que existe um $\delta=\delta\left(\varepsilon, \mathrm{t}_{0}\right)>0$ tal que, se $\|\varphi\|<\delta$ entăo $\| \mathrm{x}_{\mathrm{t}}\left(\cdot, \mathrm{t}_{\mathrm{o}}, \varphi \|<\varepsilon\right.$ para todo $\mathrm{t} \geq \mathrm{t}_{\mathrm{o}}$.

Se $t_{0} \in Z$, nada há a mostrar. Suponhamos então que $\mathrm{t}_{\mathrm{o}} \in\left(\mathrm{n}_{\mathrm{o}}-1, \mathrm{n}_{\mathrm{o}}\right)$.

Dado $\varepsilon>0$, escolhemos $\mu$ e $\delta, \delta \leq \delta\left(\mathrm{n}_{\mathrm{o}}\right), 0<\mu<\delta<\varepsilon$ tão pequenos, tais que $\|\varphi\|<\mu$ implica em $\left|x\left(t, t_{0}, \varphi\right)\right|<\delta$ para todo $t \in\left[t_{0}, n_{0}\right]$ e $\left|\mathrm{x}\left(\mathrm{n}_{\mathrm{o}}, \mathrm{t}_{\mathrm{o}}, \varphi\right)\right|<\delta$ implica em $\left|\mathrm{x}\left(\mathrm{t}_{,} \mathrm{t}_{\mathrm{o}}, \varphi\right)\right|<\varepsilon$ para todo $\mathrm{t} \geq \mathrm{t}_{\mathrm{o}}$. Ou seja, $\left\|x_{t}\left(\cdot, t_{0}, \varphi\right)\right\|<\varepsilon$ para todo $t \geq t_{0}$. Esta escolha é sempre possível devido à continuidade de $\mathrm{x}\left(\cdot, \mathrm{t}_{\mathrm{o}}, \varphi\right)$ com relação à $\varphi$.

Em se tratando de estabilidade assintótica, também temos equivalência de definições.

Definiçăo 2.2: O equilíbrio nulo de (1.3) é assintoticamente estável nos inteiros, se for estável nos inteiros e além disso existir um $\gamma=\gamma\left(\mathrm{n}_{\mathrm{o}}\right)>0$ tal que, se $\left|c_{0}\right|<\gamma$ então $x\left(t, n_{o}, c_{0}\right) \rightarrow 0$ quando $t \rightarrow \infty$, de acordo com 0 sistema (2.4).

Lema 2.2: As Definiçర̋es (1.11) e (2.2) são equivalentes. 
Demonstraçăo: É claro que a Definiçăo (1.11) implica na Definiçăo (2.2). Provemos a reciproca, ou seja, dado $t_{0} \in \mathfrak{R}$ existe um $\gamma=\gamma\left(t_{0}\right)>0$ tal que, se $\|\varphi\|<\gamma$ entăo $x\left(t, t_{0}, \varphi\right) \rightarrow 0$ quando $t \rightarrow \infty$.

Se $\mathrm{t}_{\mathrm{o}} \in Z$, nada há a mostrar. Suponhamos entăo $\mathrm{t}_{\mathrm{o}} \in\left(\mathrm{n}_{\mathrm{o}}-1, \mathrm{n}_{\mathrm{o}}\right)$.

Dado $\gamma=\gamma\left(n_{0}\right)>0$ garantido pela Definiçăo (2.2), podemos escolher $\mu$ tão pequeno, $0<\mu<\gamma$, tal que $\|\varphi\|<\mu$ implica em $\left|x\left(t, t_{0}, \varphi\right)\right|<\gamma$ para $t \in\left[t_{0}, n_{0}\right]$ e $\left|x\left(n_{0}, t_{0}, \varphi\right)\right|<\gamma$ implica em $x\left(t, n_{0}, x\left(n_{0}, t_{0}, \varphi\right)\right) \rightarrow 0$ quando $t \rightarrow \infty$, ou seja, $\left.x\left(t, t_{0}, \varphi\right)\right) \rightarrow 0$ quando $t \rightarrow \infty$. Esta escolha é possivel devido à continuidade de $\mathrm{x}\left(\cdot, \mathrm{t}_{\mathrm{o}}, \varphi\right)$ com relação à $\varphi$; e segue o resultado.

Vamos agora mostrar, sob condições específicas, que a estabilidade (estabilidade assintótica) do equilíbrio nulo da equação discreta (2.1), implica diretamente na estabilidade (estabilidade assintótica) do equilíbrio nulo da equaçăo (1.3).

Teorema 2.3: Suponhamos $f$ Lipschitziana nas variáveis $(x, y)$ em $\mathfrak{R} \times \mathfrak{R}^{2 \mathrm{n}}$. Se o equilíbrio nulo da equação discreta (2.1) é estável, então o equilíbrio nulo da equação (1.3) é também estável.

Demonstração: Por hipótese, dado $\varepsilon>0$ existe $\delta=\delta\left(\mathrm{n}_{0}, \varepsilon\right)>0$ tal que, se $\left|c_{0}\right|<\delta$ então $\left|c_{n}\left(c_{0}\right)\right|<\varepsilon$ para todo $n \in N$. E para cada restriçăo $x(t)$ da soluçăo em $\left[n_{0}+n, n_{0}+n+1\right), n \in N$, temos que:

$$
x(t)=c_{n}+\int_{n_{0}+n}^{t} f\left(s, x(s), c_{n}\right) d s
$$


Assim, como f é Lipschitziana em ( $x, y)$ com constante de Lipschitz $k$, segue que:

$$
|x(t)| \leq(1+k)\left|c_{n}\right|+k \int_{n_{0}+n}^{t}|x(s)| d s,
$$

e pela Desigualdade de Gronwall, $|x(t)| \leq(1+k)\left|c_{n}\right| e^{k}=M\left|c_{n}\right|$, onde $\mathrm{M}=(1+\mathrm{k}) \mathrm{e}^{\mathrm{k}}$.

Logo, sempre que $\left|c_{n}\left(c_{0}\right)\right|<\varepsilon / M$, temos que $|x(t)|<\varepsilon$ para $t \in\left[n_{0}+n, n_{0}+n+1\right)$, para todo $n \in N$.

Com este resultado, usando a continuidade de $\mathrm{x}\left(\cdot, \mathrm{t}_{0}, \varphi\right)$ com relaçăo à $\varphi$ e a hipótese, dado $\varepsilon>0$ podemos escolher $\mu$ e $\delta, \delta \leq \delta\left(n_{0}\right)$, $0<\mu<\delta<\varepsilon$ tăo pequenos tais que $\|\varphi\|<\mu$ implica em $\left|x\left(t, t_{0}, \varphi\right)\right|<\delta$ para $\mathrm{t} \in\left[\mathrm{t}_{0}, \mathrm{n}_{\mathrm{o}}\right]$ e $\left|\mathrm{x}\left(\mathrm{n}_{\mathrm{o}}, \mathrm{t}_{\mathrm{o}}, \varphi\right)\right|=\left|\mathrm{c}_{\mathrm{o}}\right|<\delta$ implica em $\left|\mathrm{c}_{\mathrm{n}}\left(\mathrm{c}_{\mathrm{o}}\right)\right|<\varepsilon / \mathrm{M}<\varepsilon$, para todo $n \in N$, que por sua vez implica em $\left|x\left(t, t_{0}, \varphi\right)\right|<\varepsilon$, para todo $t \geq t_{0}$. $E$ temos o resultado desejado.

Teorema 2.4: Com as hipóteses do Teorema (2.3), se o equilíbrio nulo da equaçáo (2.1) é assintoticamente estável, então o equilíbrio nulo da equaçăo (1.3) é também assintoticamente estável.

Demonstração: Por hipótese, dado $\mathrm{n}_{\mathrm{o}} \in \mathrm{N}$, existe $\gamma=\gamma\left(\mathrm{n}_{\mathrm{o}}\right)>0$ tal que se $\left|c_{0}\right|<\gamma$, entăo $c_{n}\left(c_{0}\right) \rightarrow 0$ quando $n \rightarrow \infty$.

Como no Teorema (2.3), dado $t_{0} \in \mathfrak{R}$, podemos escolher $\mu$ e $\gamma$, $\gamma \leq \gamma\left(\mathrm{n}_{\mathrm{o}}\right), \quad 0<\mu<\gamma$ tăo pequenos tais que $\|\varphi\|<\mu$ implica em 
$\left|x\left(t, t_{0}, \varphi\right)\right|<\gamma$ para $t \in\left[t_{0}, n_{0}\right]$ e $\left|c_{0}\right|<\gamma$ implica que $c_{n}\left(c_{0}\right) \rightarrow 0$ quando $n \rightarrow \infty$, que por sua vez implica que $x\left(t, t_{0}, \varphi\right) \rightarrow 0$ quando $t \rightarrow \infty$.

\subsection{Equaçăo Autơnoma nos Inteiros}

Neste parágrafo, abordamos um caso particular da equação (1.3) dada por:

$$
\begin{aligned}
& x^{\prime}(t)=f(x(t), x([t])) \quad \text { q.t.p., } \quad t \geq t_{0} \\
& x_{t_{0}}=\varphi
\end{aligned}
$$

Apesar da função $f$ não depender diretamente da variável $t$, a equaçăo (2.5) não é autônoma pois, como vimos, é equivalente à equação (1.2) para uma g apropriada.

Observamos que, encontrar solução de (2.5) com valor inicial em $t_{0}$, é equivalente à encontrar soluçăo de (2.5) com valor inicial dado em $t_{0}+n$, onde n é um inteiro não negativo, como veremos.

Definição 2.3: Dizemos que a equaçăo diferencial funcional (1.2) é autónoma em $\mathrm{A} \subset \Re_{+}$, quando

$$
x\left(t+\tau, t_{0}, \varphi\right)=x\left(t, t_{0}, x_{t_{0}+\tau}\left(\cdot, t_{0}, \varphi\right)\right), \quad \text { para todo } \tau \in A
$$

Lema 2.5: A equaçăo (2.5) é autônoma nos inteiros, isto é,

$$
x\left(t+n, t_{0}, \varphi\right)=x\left(t, t_{0}, x_{t_{0}+n}\left(\cdot, t_{0}, \varphi\right)\right), \text { para todo } n \in Z_{+} .
$$


Demonstraçăo: Se $x\left(t, t_{0}, x_{t_{0+q}}\left(\cdot, t_{0}, \varphi\right)\right)$ é soluçắo de (2.5), mostremos que $\psi(t)=x\left(t+n, t_{0}, \varphi\right)$ também satisfaz (2.5). De fato:

$$
\psi^{\prime}(t)=x^{\prime}\left(t+n, t_{0}, \varphi\right)=f\left(x\left(t+n, t_{0}, \varphi\right), x\left([t]+n, t_{0}, \varphi\right)\right)=f(\psi(t), \psi([t])) .
$$

E no intervalo $\left[t_{0}-1, t_{0}\right]$ as funçס̌es coincidem, pois para $\theta \in[-1,0]$ temos:

$$
\begin{aligned}
& \psi\left(\mathrm{t}_{\mathrm{o}}+\theta\right)=\mathrm{x}\left(\mathrm{t}_{\mathrm{o}}+\theta+\mathrm{n}, \mathrm{t}_{\mathrm{o}}, \varphi\right)=\mathrm{x}_{\mathrm{t}_{\mathrm{o}}+n}\left(\cdot, \mathrm{t}_{\mathrm{o}}, \varphi\right)(\theta) \\
& \text { e } \quad \mathrm{x}\left(\mathrm{t}_{\mathrm{o}}+\theta, \mathrm{t}_{\mathrm{o}}, \mathrm{x}_{\mathrm{t}_{\mathrm{o}+\mathrm{n}}}\left(\cdot, \mathrm{t}_{\mathrm{o}}, \varphi\right)\right)=\mathrm{x}_{\mathrm{t}_{\mathrm{o}+\mathrm{n}}}\left(\cdot, \mathrm{t}_{\mathrm{o}}, \varphi\right)(\theta) .
\end{aligned}
$$

Dada a unicidade de soluçăo, temos a igualdade desejada.

Uma propriedade básica da equação (2.5), sendo autônoma nos inteiros, é a seguinte: se $x(t)$ é solução de (2.5) definida no intervalo $\left[t_{0}, b\right)$, então para todo inteiro năo negativo $n$, a função $x(t-n)$ é soluçăo de (2.5) definida no intervalo $\left[t_{0}+n, b+n\right)$.

E em virtude dos Lemas (2.1), (2.2) e (2.5), constatamos que no estudo da estabilidade do equilíbrio nulo, é suficiente tomar como instante inicial um número inteiro, e como a equaçăo (2.5) é autônoma nos inteiros, basta tomar $t_{0}=0$. Esta escolha facilita bastante a relação entre a equação (2.5) e a sua equaçăo discreta associada, que por sua vez tem a seguinte característica:

Lema 2.6: A equaçăo discreta associada à equaçăo (2.5) é autônoma. 
Demonstração: A equaçåo discreta é dada por $c_{n+1}=h\left(n, c_{n}\right)$, $\mathrm{n}=0,1, \cdots$, conforme (2.1).

Provemos que $h(n, d)=h(m, d)$ quando $c_{n}=c_{m}=d$, para $n, m \in N$, arbitrários. Basta provar que $c_{n+1}=c_{m+1}$.

Sabemos que $c_{j+1}=x\left(n_{0}+j+1, t_{0}, \varphi\right), j=0,1, \cdots$, e como a equaçăo (2.5) é autônoma nos inteiros e $c_{n}=c_{m}$, segue que:

$$
\begin{aligned}
\mathrm{c}_{\mathrm{m}+1} & =\mathrm{x}\left(\mathrm{n}_{\mathrm{o}}+\mathrm{m}+1, \mathrm{t}_{\mathrm{o}}, \varphi\right)=\mathrm{x}\left(\mathrm{n}_{\mathrm{o}}+1, \mathrm{t}_{\mathrm{o}}, \mathrm{x}_{\mathrm{t}_{\mathrm{o}}+\mathrm{m}}\left(., \mathrm{t}_{\mathrm{o}}, \varphi\right)\right)= \\
& =\mathrm{x}\left(\mathrm{n}_{\mathrm{o}}+1, \mathrm{t}_{\mathrm{o}}, \mathrm{x}_{\mathrm{t}_{\mathrm{o}}+\mathrm{n}}\left(\cdot, \mathrm{t}_{\mathrm{o}}, \varphi\right)\right)=\mathrm{x}\left(\mathrm{n}_{\mathrm{o}}+\mathrm{n}+1, \mathrm{t}_{\mathrm{o}}, \varphi\right)=\mathrm{c}_{\mathrm{n}+1},
\end{aligned}
$$

e portanto, temos a igualdade desejada. 


\section{Capitulo 3}

\section{ESTUDO DA ESTABILIDADE E ESTABILIDADE ASSINTÓTICA USANDO FUNÇÕES DICOTÓMICAS}

\subsection{A Equação Discreta (1.1)}

Neste parágrafo, apresentamos o uso de Funções Dicotômicas para garantir a estabilidade e estabilidade assintótica da solução nula da equação discreta não autônoma (1.1), generalizando a teoria apresentada por Carvalho e Ferreira [5] e Carvalho [3], para o caso autônomo.

Teorema 3.1: Suponhamos que $u, v: \mathfrak{R}_{+} \rightarrow \mathfrak{R}_{+}$sejam funções contínuas, não decrescentes com $u(0)=v(0)=0, u(x)$ e $v(x)$ positivas para $\mathrm{x}>0$.

Se existir uma função $\mathrm{V}: \mathfrak{R} \times \mathfrak{R}^{\mathrm{n}} \rightarrow \mathfrak{R}$ definida positiva, dicotômica com relação à equação discreta (1.1), satisfazendo

$$
\mathrm{u}(|\mathrm{x}|) \leq \mathrm{V}(\mathrm{t}, \mathrm{x}) \leq \mathrm{v}(|\mathrm{x}|), \quad \mathrm{t} \in \mathfrak{R}, \mathrm{x} \in \mathfrak{R}^{\mathrm{n}},
$$

então o equilíbrio nulo de (1.1) é estável.

Demonstração: Para simplificar, usaremos a notação $\mathrm{x}_{\mathrm{n}}\left(\mathrm{x}_{\mathrm{o}}\right)$ para a soluçăo de (1.1). 
Seja $\Omega$ uma vizinhança da origem em $\mathfrak{R}^{\mathrm{n}}$, onde $\mathrm{V}$ é dicotômica com relaçăo à equaçăo (1.1).

Para qualquer $\varepsilon>0$, existe um $\delta_{0}=\delta_{0}(\varepsilon), 0<\delta_{0}<\varepsilon$ tal que $v\left(\delta_{0}\right)<u(\varepsilon)$.

Tomemos $\varepsilon_{0}$ e $\delta_{0}$ suficientemente pequenos, $\delta_{0}<\varepsilon_{0}<\varepsilon$ com $\mathrm{v}\left(\delta_{0}\right)<\mathrm{u}\left(\varepsilon_{0}\right)$, tais que $|\mathrm{x}|<\varepsilon_{0}$ implica que $\mathrm{x} \in \Omega$.

Dado $\mathrm{n}_{\mathrm{o}} \in \mathrm{Z}$, a continuidade de $\mathrm{x}_{\mathrm{n}}\left(\mathrm{x}_{\mathrm{o}}\right)$ com relaçăo à $\mathrm{x}_{0}, a$ continuidade de $\mathrm{V}$ e o fato de $\mathrm{V}(\mathrm{t}, 0)=0$, garantem a existência de um $\delta_{1}=\delta_{1}\left(\varepsilon_{0}, \mathrm{n}_{\mathrm{o}}\right)>0, \delta_{1}<\varepsilon_{0}$, tal que se $\left|\mathrm{x}_{\mathrm{o}}\right|<\delta_{1}$ então $\mathrm{V}\left(\mathrm{n}, \mathrm{x}_{\mathrm{n}}\left(\mathrm{x}_{\mathrm{o}}\right)\right)<\mathrm{u}\left(\varepsilon_{\mathrm{o}}\right)$, para $n$ variando entre $n_{0}$ e $n_{0}+N\left(n_{0}\right) ; N\left(n_{0}\right)$ dado pela Definição de dicotomia (1.6).

Seja $\delta=\min \left\{\delta_{0}, \delta_{1}\right\}$. Mostremos que se $\left|x_{0}\right|<\delta$ então $\mathrm{V}\left(\mathrm{n}, \mathrm{x}_{\mathrm{n}}\left(\mathrm{x}_{\mathrm{o}}\right)\right)<\mathrm{u}\left(\varepsilon_{\mathrm{o}}\right)$, para todo $\mathrm{n} \geq \mathrm{n}_{\mathrm{o}}$.

Suponhamos, por absurdo, que exista um primeiro instante $\mathrm{n}_{1}>\mathrm{n}_{\mathrm{o}}+\mathrm{N}\left(\mathrm{n}_{\mathrm{o}}\right)$ tal que:

$$
\begin{aligned}
& \mathrm{V}\left(\mathrm{n}_{1}, \mathrm{x}_{\mathrm{n}_{1}}\left(\mathrm{x}_{\mathrm{o}}\right)\right) \geq \mathrm{u}\left(\varepsilon_{\mathrm{o}}\right) \\
& \text { e } \quad \mathrm{V}\left(\mathrm{n}, \mathrm{x}_{\mathrm{n}}\left(\mathrm{x}_{\mathrm{o}}\right)\right)<\mathrm{u}\left(\varepsilon_{\mathrm{o}}\right), \text { para } \mathrm{n}<\mathrm{n}_{1} .
\end{aligned}
$$

Isto implica que $\mathrm{V}\left(\mathrm{n}_{1}, \mathrm{x}_{\mathrm{n}_{1}}\left(\mathrm{x}_{\mathrm{o}}\right)\right)-\mathrm{V}\left(\mathrm{n}_{1}-1, \mathrm{x}_{\mathrm{n}_{1}-1}\left(\mathrm{x}_{\mathrm{o}}\right)\right)>0$.

Assim, como V é dicotômica com relação à equação (1.1), existe $\mathrm{k}=\mathrm{k}\left(\mathrm{n}_{\mathrm{o}}, \mathrm{n}_{1}\right), \mathrm{k} \in \mathrm{Z}_{+}, \quad 2 \leq \mathrm{k} \leq \mathrm{n}_{1}-\mathrm{n}_{\mathrm{o}}$, tal que :

$$
\mathrm{x}_{\mathrm{n}_{1}-\mathrm{k}}\left(\mathrm{x}_{\mathrm{o}}\right) \in \bar{\Omega}_{+}^{1}\left(\mathrm{n}_{1}, \mathrm{k}\right) \subset \bigcup_{\mathrm{j}=2}^{\mathrm{k}} \Omega_{\mathrm{o}-(}^{\mathrm{j}}\left(\mathrm{n}_{1}, \mathrm{k}\right) .
$$


Segue que $V\left(n_{1}, x\left(n_{1}, n_{1}-k, x_{n_{1}-k}\left(x_{0}\right)\right)-V\left(n_{1}-j, x\left(n_{1}-j, n_{1}-k, x_{n_{1}-k}\left(x_{0}\right)\right) \leq 0\right.\right.$, para algum $j, j=2, \cdots, k$, ou seja,

$$
V\left(n_{1}, x_{n_{1}}\left(x_{o}\right)\right) \leq V\left(n_{1}-j, x_{n_{1}-j}\left(x_{0}\right)\right)<u\left(\varepsilon_{o}\right) ; \text { para algum } j, j=2, \cdots, k \text {, }
$$

em contradiçăo à nossa suposiçăo inicial.

Logo, se $\left|x_{0}\right|<\delta$ então $V\left(n, x_{n}\left(x_{0}\right)\right)<u\left(\varepsilon_{0}\right)$, para $n \geq n_{0}$.

Por hipótese, temos que:

$$
\mathrm{u}\left(\left|\mathrm{x}_{\mathrm{n}}\left(\mathrm{x}_{\mathrm{o}}\right)\right|\right) \leq \mathrm{V}\left(\mathrm{n}, \mathrm{x}_{\mathrm{n}}\left(\mathrm{x}_{\mathrm{o}}\right)\right)<\mathrm{u}\left(\varepsilon_{\mathrm{o}}\right) \text {, para } \mathrm{n} \geq \mathrm{n}_{\mathrm{o}} .
$$

Como u é não decrescente, segue que $\left|\mathrm{x}_{\mathrm{n}}\left(\mathrm{x}_{\mathrm{o}}\right)\right|<\varepsilon_{\mathrm{o}}<\varepsilon$ para $\mathrm{n} \geq \mathrm{n}_{\mathrm{o}}$. Portanto, o equilíbrio nulo de (1.1) é estável.

A função $p$ dada no ítem (iv) e exigida na Definição de dicotomia estrita (1.7), é fundamental para a prova do próximo Teorema. Este fato é constatado no seguinte resultado:

Lema 3.2: Sejam u,ve V como no Teorema (3.1). Além disso, suponhamos que $\mathrm{V}$ seja estritamente dicotômica com relação à equação (1.1). Então, sempre que para um determinado $n_{1}, n_{1} \geq n_{0}+N\left(n_{0}\right)$, tivermos $\mathrm{V}\left(\mathrm{n}_{1}, \mathrm{x}_{\mathrm{n}_{1}}\left(\mathrm{x}_{\mathrm{o}}\right)\right)>\beta$ e $\Delta_{1} \mathrm{~V}\left(\mathrm{n}_{1}, \mathrm{x}_{\mathrm{n}_{1}}\left(\mathrm{x}_{\mathrm{o}}\right)\right) \geq 0$, existirá um $\rho=\rho(\beta)>0$, tal que:

$$
V\left(n_{1}, x_{n_{1}}\left(x_{0}\right)\right)-V\left(n_{1}-j, x_{n_{1}-j}\left(x_{o}\right)\right)<-\rho \text {, para algum } j, j=2, \cdots, k \text {, }
$$

onde k e $N\left(n_{o}\right)$ são como na Definiçăo (1.6). 
Demonstraçăo: Pelo Teorema (3.1), existem $\delta_{1}>0$ e $\delta_{2}>0$ tais que se $\left|x_{0}\right|<\delta_{1}$ entăo $V\left(n, x_{n}\left(x_{0}\right)\right)<\delta_{2}$, para $n \geq n_{0}$. Se $V\left(n_{1}, x_{n_{1}}\left(x_{0}\right)\right)>\beta$, segue que $\beta<V\left(n_{1}, x_{n_{1}}\left(x_{0}\right)\right)<\delta_{2}$.

Se $V\left(n_{1}, x_{n_{1}}\left(x_{0}\right)-V\left(n_{1}-1, x_{n_{1}-1}\left(x_{0}\right)\right) \geq 0\right.$, como $V$ é estritamente dicotômica com relaçăo à equaçăo (1.1), existe $k=k\left(n_{0}, n_{1}\right), k \in Z_{+}$, $2 \leq \mathrm{k} \leq \mathrm{M}$, tal que:

$$
\begin{gathered}
\mathrm{x}_{\mathrm{n}_{1}-\mathrm{k}}\left(\mathrm{x}_{\mathrm{o}}\right) \in\left[\bar{\Omega}_{+}^{1}\left(\mathrm{n}_{1}, \mathrm{k}\right) \cup \Omega_{\mathrm{o}}^{1}\left(\mathrm{n}_{1}, \mathrm{k}\right)\right]^{*} \subset \bigcup_{j=2}^{\mathrm{k}} \Omega_{\mathrm{p} .}^{\mathrm{j}}\left(\mathrm{n}_{1}, \mathrm{k}\right), \text { isto é, } \\
\mathrm{p}\left(\mathrm{V}\left(\mathrm{n}_{1}, \mathrm{x}_{\mathrm{n}_{1}}\left(\mathrm{x}_{\mathrm{o}}\right)\right)<\mathrm{V}\left(\mathrm{n}_{1}-\mathrm{j}, \mathrm{x}_{\mathrm{n}_{1}-\mathrm{j}}\left(\mathrm{x}_{\mathrm{o}}\right)\right), \text { para algum } \mathrm{j}, \mathrm{j}=2, \cdots, \mathrm{k} .\right. \\
\text { Seja } 0<\rho(\beta)<\min \left\{\mathrm{p}(\mathrm{y})-\mathrm{y}: \mathrm{y} \in\left[\beta, \delta_{2}\right]\right\} . \text { Assim, segue que: } \\
\mathrm{V}\left(\mathrm{n}_{1}-\mathrm{j}, \mathrm{x}_{\mathrm{n}_{1}-\mathrm{j}}\left(\mathrm{x}_{\mathrm{o}}\right)\right)>\mathrm{p}\left(\mathrm{V}\left(\mathrm{n}_{1}, \mathrm{x}_{\mathrm{n}_{1}}\left(\mathrm{x}_{\mathrm{o}}\right)\right)>\mathrm{V}\left(\mathrm{n}_{1}, \mathrm{x}_{\mathrm{n}_{1}}\left(\mathrm{x}_{\mathrm{o}}\right)\right)+\rho(\beta),\right. \text { portanto, } \\
\mathrm{V}\left(\mathrm{n}_{1}, \mathrm{x}_{\mathrm{n}_{1}}\left(\mathrm{x}_{\mathrm{o}}\right)\right)-\mathrm{V}\left(\mathrm{n}_{1}-\mathrm{j}, \mathrm{x}_{\mathrm{n}_{1}-\mathrm{j}}\left(\mathrm{x}_{\mathrm{o}}\right)\right)<-\rho(\beta), \text { para algum } \mathrm{j}, \mathrm{j}=2, \cdots, \mathrm{k} .
\end{gathered}
$$

Teorema 3.3: Seja V satisfazendo todas as hipóteses do Teorema (3.1) e estritamente dicotômica com relação à equação (1.1). Então, o equilíbrio nulo de (1.1) é assintoticamente estável.

Demonstração: Sejam $\delta$ e $u(\varepsilon)$ como no Teorema (3.1). Tomemos $\gamma \leq \delta$ e mostremos que se $\left|x_{0}\right|<\gamma$ então, $x_{n}\left(x_{0}\right) \rightarrow 0$ quando $n \rightarrow \infty$.

Com relação à $x_{n}\left(x_{0}\right)$, existem duas alternativas: 
(i) existe um $n_{1}>n_{0}+N$ tal que $\Delta_{1} V\left(n, x_{a}\left(x_{0}\right)\right) \leq 0$ para todo $n \geq n_{1}$ ou,

(ii) existe uma seqüência $\left(n_{j}\right)$ tal que, para todo termo $n_{j}$ temos $\Delta_{1} \mathrm{~V}\left(\mathrm{n}_{\mathrm{j}}, \mathrm{x}_{\mathrm{n}_{\mathrm{j}}}\left(\mathrm{x}_{\mathrm{o}}\right)\right)>0$.

caso (i): Se existir um $\tau>0$ tal que $\Delta_{1} V\left(n, x_{n}\left(x_{0}\right)\right)<-\tau$ para todo $\mathrm{n} \geq \mathrm{n}_{1}$, temos que:

$$
\begin{aligned}
\mathrm{V}\left(\mathrm{n}, \mathrm{x}_{\mathrm{n}}\left(\mathrm{x}_{\mathrm{o}}\right)\right)= & \mathrm{V}\left(\mathrm{n}-1, \mathrm{x}_{\mathrm{n}-1}\left(\mathrm{x}_{\mathrm{o}}\right)\right)+\left[\mathrm{V}\left(\mathrm{n}, \mathrm{x}_{\mathrm{n}}\left(\mathrm{x}_{\mathrm{o}}\right)\right)-\mathrm{V}\left(\mathrm{n}-1, \mathrm{x}_{\mathrm{n}-1}\left(\mathrm{x}_{\mathrm{o}}\right)\right)\right]< \\
& <\mathrm{V}\left(\mathrm{n}-1, \mathrm{x}_{\mathrm{n}-1}\left(\mathrm{x}_{\mathrm{o}}\right)\right)-\tau .
\end{aligned}
$$

Uma aplicação sucessiva deste processo, leva-nos à seguinte desigualdade:

$$
\mathrm{V}\left(\mathrm{n}+\mathrm{m}, \mathrm{x}_{\mathrm{n}+\mathrm{m}}\left(\mathrm{x}_{\mathrm{o}}\right)\right)<\mathrm{V}\left(\mathrm{n}-1, \mathrm{x}_{\mathrm{n}-1}\left(\mathrm{x}_{\mathrm{o}}\right)\right)-(\mathrm{m}+1) \tau, \mathrm{m} \in \mathrm{N},
$$

e portanto segue que $V\left(n, x_{n}\left(x_{0}\right)\right) \rightarrow-\infty$ quando $n \rightarrow \infty$, o que contradiz a hipótese de $\mathrm{V}$ ser uma função definida positiva.

Se tal fato não ocorrer, da hipótese do caso (i) temos que $\mathrm{V}\left(\mathrm{n}, \mathrm{x}_{\mathrm{n}}\left(\mathrm{x}_{\mathrm{o}}\right)\right)$ é decrescente para $n \geq n_{1}$. Da limitaçăo de $V$, segue que $V\left(n, x_{n}\left(x_{0}\right)\right) \rightarrow c$ quando $\mathrm{n} \rightarrow \infty$, para alguma constante $\mathrm{c}$ não negativa.

Assim, $\quad \mathrm{V}\left(\mathrm{n}, \mathrm{x}_{\mathrm{n}}\left(\mathrm{x}_{\mathrm{o}}\right)\right)-\mathrm{V}\left(\mathrm{n}-\mathrm{j}, \mathrm{x}_{\mathrm{n}-\mathrm{j}}\left(\mathrm{x}_{\mathrm{o}}\right)\right) \rightarrow 0$ quando $\mathrm{n} \rightarrow \infty$, para todo $j, \mathrm{j}=1, \cdots, \mathrm{M}, \mathrm{M}$ da definiçăo de dicotomia estrita.

Logo, $x_{n-M}\left(x_{0}\right) \rightarrow \bigcap_{j=1}^{M} \Omega_{0}^{j}(n, M)$ quando $n \rightarrow \infty$ e pelo ítem (iii) da Definiçăo (1.7), segue que $x_{n}\left(x_{0}\right) \rightarrow 0$ quando $n \rightarrow \infty$. 
caso (ii): Vamos definir uma nova seqüência $\left(m_{1}\right)$, subsequééncia de $\left(n_{j}\right)$, da seguinte forma: $m_{1}=n_{1} ; m_{2}$ é o primeiro elemento da seqüência $\left(n_{j}\right)$, digamos $n_{j_{0}}$, tal que $n_{j_{o}} \geq m_{1}+M$. Em geral, definimos $m_{k}, k \geq 2$ como o primeiro elemento da seqüência $\left(n_{j}\right)$, digamos $n_{J_{r}}$, tal que $\mathrm{n}_{\mathrm{j}_{\mathrm{r}}} \geq \mathrm{m}_{\mathrm{k}-\mathrm{l}}+\mathrm{M}$.

Assim, para todo elemento da seqüência $\left(m_{j}\right)$, temos que $\Delta_{1} \mathrm{~V}\left(\mathrm{~m}_{\mathrm{j}}, \mathrm{x}_{\mathrm{m}_{\mathrm{j}}}\left(\mathrm{x}_{\mathrm{o}}\right)\right)>0$.

Definimos agora:

$$
\begin{aligned}
& \beta_{j}=\max \left\{V\left(n, x_{n}\left(x_{o}\right)\right): m_{j-1} \leq n \leq m_{j}\right\}, j=2,3, \cdots \\
& j^{*}=\min \left\{n: m_{j-1} \leq n \leq m_{j} \text { e } \beta_{j}=V\left(n, x_{n}\left(x_{o}\right)\right)\right\}, j=2,3, \cdots
\end{aligned}
$$

Provemos que a seqüência $\left(\beta_{j}\right)$ é estritamente decrescente.

De fato, temos duas situações a considerar para j arbitrário:
(i) $\mathrm{j}^{*}=\mathrm{m}_{\mathrm{j}-1}$
(ii) $j^{*}>m_{j-1}$

caso (i): Neste caso temos, por construção da seqüência, que $\Delta_{1} \mathrm{~V}\left(\mathrm{j}^{*}, \mathrm{x}_{\mathrm{j}^{*}}\left(\mathrm{x}_{\mathrm{o}}\right)\right)>0$ e assim, pela hipótese de dicotomia estrita, existe um $k=k\left(n_{o}, j^{*}\right), 2 \leq k \leq j^{*}-n_{0}$, tal que:

$$
\begin{gathered}
x_{j^{*}-k}\left(x_{o}\right) \in\left[\bar{\Omega}_{+}^{1}\left(j^{*}, k\right) \cup \Omega_{o}^{1}\left(j^{*}, k\right)\right] \subset \bigcup_{j=2}^{k} \Omega_{p-}^{j}\left(j^{*}, k\right), \text { ou seja, } \\
p\left(V\left(j^{*}, x_{j^{*}}\left(x_{0}\right)\right)<V\left(j^{*}-j_{0}, x_{j^{*}-j_{0}}\left(x_{0}\right)\right), \text { para algum } j_{0}, j_{0}=2, \cdots, k\left(j^{*}\right) .\right.
\end{gathered}
$$


Temos que $\left(j^{*}-j_{o}\right) \in\left[m_{j-2}, m_{j-1}\right]$, considerando que $j_{o} \leq k\left(j^{*}\right) \leq M e$ que cada faixa tem amplitude maior ou igual à M. Assim,

$$
\beta_{j}=V\left(j^{*}, x_{j}\left(x_{0}\right)\right)<p\left(V\left(j^{*}, x_{j}\left(x_{0}\right)\right)<V\left(j^{*}-j_{0}, x_{j-j_{0}}\left(x_{0}\right)\right) \leq \beta_{j-1} .\right.
$$

caso (ii): Neste caso, obrigatoriamente temos que $\mathrm{V}\left(\mathrm{j}^{*}, \mathrm{x}_{\mathrm{j}}\left(\mathrm{x}_{\mathrm{o}}\right)\right)>\mathrm{V}\left(\mathrm{j}^{*}-1, \mathrm{x}_{j_{-1}}\left(\mathrm{x}_{\mathrm{o}}\right)\right)$, pois no caso inverso o valor mínimo $\mathrm{j}$ no qual $\beta_{j}$ é assumido seria $\left(j^{*}-1\right)$ e não mais $j^{*}$; o que contraria a suposiçăo inicial. E portanto, o resultado segue análogo ao caso (i).

Observemos que 0 caso $\mathrm{j}^{*}=\mathrm{m}_{\mathrm{j}}$ não ocorre, pois senão $\mathrm{V}\left(\mathrm{j}^{*}, \mathrm{x}_{j}\left(\mathrm{x}_{\mathrm{o}}\right)\right)>\mathrm{V}\left(\mathrm{j}^{*}-1, \mathrm{x}_{\mathrm{j}^{*}-1}\left(\mathrm{x}_{\mathrm{o}}\right)\right)$ e pela hipótese de dicotomia estrita, segue que:

$$
\beta_{j}=V\left(j^{*}, x_{j^{*}}\left(x_{0}\right)\right)<p\left(V\left(j^{*}, x_{j^{*}}\left(x_{0}\right)\right)<V\left(j^{*}-j_{0}, x_{j^{*}-j_{0}}\left(x_{o}\right)\right)\right.
$$

para algum $\mathrm{j}_{\mathrm{o}}, \mathrm{j}_{\mathrm{o}}=2, \cdots, \mathrm{k}$.

Mas como $j_{0} \leq k\left(j^{*}\right) \leq M$ e $j^{*}=m_{j}$, temos que $\left(j^{*}-j_{0}\right) \in\left[m_{j-1}, m_{j}\right]$ que tem como máximo valor de $\mathrm{V}\left(\mathrm{n}, \mathrm{x}_{\mathrm{n}}\left(\mathrm{x}_{\mathrm{o}}\right)\right)$ o elemento $\beta_{\mathrm{j}}$. Segue então que $\mathrm{V}\left(\mathrm{j}^{*}-\mathrm{j}_{0}, \mathrm{x}_{j^{*}-\mathrm{j}_{0}}\left(\mathrm{x}_{\mathrm{o}}\right)\right) \leq \beta_{\mathrm{j}}$, que é uma contradição para (3.1).

Portanto, em todos os casos temos que $\beta_{j}<\beta_{j-1}$, ou seja, $\left(\beta_{j}\right)$ é uma seqüência estritamente decrescente. Provemos agora que $\beta_{j} \rightarrow 0$ quando $j \rightarrow \infty$. Suponhamos que $\beta, \beta$ quando $j \rightarrow \infty, \beta>0$.

Temos duas situações a considerar:

(i) existem infinitos termos $j^{*}$, tais que $j^{*} \in\left(m_{j-1}, m_{\jmath}\right)$,

(ii) existem infinitos termos $\mathrm{j}^{*}$, tais que $\mathrm{j}^{*}=\mathrm{m}_{\mathrm{j}-1}$. 
Caso (i): Consideremos a subseqüência de $\left(\beta_{j}\right)$, que usaremos a mesma notaçăo, tal que $\mathrm{j}^{*}$ é ponto interior do intervalo $\left[\mathrm{m}_{\mathrm{j}-1}, \mathrm{~m}_{\mathrm{j}}\right]$, isto é, necessariamente $\Delta_{1} \mathrm{~V}\left(\mathrm{j}^{*}, \mathrm{x}_{j}\left(\mathrm{x}_{\mathrm{o}}\right)\right)>0$ e $\mathrm{V}\left(\mathrm{j}^{*}, \mathrm{x}_{j^{*}}\left(\mathrm{x}_{\mathrm{o}}\right)\right)>\beta$ para todo índice j' da nova seqüência.

Pelo Lema (3.2) segue que existe um $\rho=\rho(\beta)>0$, tal que:

$$
\mathrm{V}\left(\mathrm{j}^{*}, \mathrm{x}_{j^{*}}\left(\mathrm{x}_{\mathrm{o}}\right)\right)-\mathrm{V}\left(\mathrm{j}^{*}-\mathrm{j}_{\mathrm{o}}, \mathrm{x}_{\mathrm{j}^{*}-\mathrm{j}_{\mathrm{o}}}\left(\mathrm{x}_{\mathrm{o}}\right)\right)<-\rho(\beta) \text {, para algum } \mathrm{j}_{\mathrm{o}}, \mathrm{j}_{\mathrm{o}}=2, \cdots, \mathrm{k} .
$$

Logo, $\beta_{j}<\beta_{j-1}-\rho(\beta)$, para todo índice $j$ da subseqüência, ou seja: $\beta_{\mathrm{j}+1}<\beta_{\mathrm{j}}-\rho<\beta_{j-1}-2 \rho$, e uma aplicação sucessiva deste processo, leva-nos à seguinte desigualdade: $\beta_{j+m}<\beta_{j}-m \rho, m \in N$.

Logo, $\beta_{j+m} \rightarrow-\infty$ quando $m \rightarrow \infty$, e assim $\beta_{j} \rightarrow-\infty$ quando $j \rightarrow \infty, 0$ que contradiz a nossa suposição. Portanto, $\beta=0$ e segue que $\beta_{j} \rightarrow 0$ quando $\mathrm{j} \rightarrow \infty$.

Caso (ii): Temos então uma subseqüência de $\left(\beta_{\mathrm{j}}\right)$, onde $\mathrm{j}^{*}=\mathrm{m}_{\mathrm{j}-1}$ para todo índice da subseqüência, para os quais, por construção da seqüência $\left(\beta_{\mathrm{j}}\right)$, temos que $\Delta_{1} \mathrm{~V}\left(\mathrm{j}^{*}, \mathrm{x}_{\mathrm{j}^{*}}\left(\mathrm{x}_{\mathrm{o}}\right)\right)>0$. Assim, segue análogo ao caso (i).

Portanto, em ambos os casos, temos que $\beta_{\mathrm{j}} \rightarrow 0$ quando $\mathrm{j} \rightarrow \infty$.

$E$ como $u\left(\left|x_{n}\left(x_{0}\right)\right|\right) \leq V\left(n, x_{n}\left(x_{0}\right)\right), u(0)=0$ e $V\left(n, x_{n}\left(x_{o}\right)\right) \rightarrow 0$ quando $\mathrm{n} \rightarrow \infty$, concluímos que $\left.\mathrm{x}_{\mathrm{n}}\left(\mathrm{x}_{\mathrm{o}}\right)\right) \rightarrow 0$ quando $\mathrm{n} \rightarrow \infty$ e portanto, o equilíbrio nulo de (1.1) é assintoticamente estável. 


\subsection{A Equaçăo Diferenclal (1.3)}

Neste parágrafo, enunciamos e demonstramos os teoremas que garantem estabilidade e estabilidade assintótica da soluçăo nula da equaçăo diferencial (1.3), utilizando a noçăo de Funçăo Dicotômica.

Para tanto, vamos supor que $f: \Re \times \Re^{2 \mathfrak{n}} \rightarrow \Re^{\mathrm{n}}$ em (1.3), leve conjuntos limitados em conjuntos limitados.

A teoria a ser desenvolvida é uma adaptação ao trabalho de Bená [2] que trabalha com equações diferenciais funcionais retardadas não autônomas do tipo:

$$
\begin{aligned}
& x^{\prime}(t)=g\left(t, x_{t}\right), \quad t \geq t_{0} \\
& x_{t_{0}}=\varphi
\end{aligned}
$$

com a hipótese de continuidade de g, entre outras.

Lemas adicionais são necessários para garantir a validade da propriedade de dicotomia nos pontos de máximo local, onde $\mathrm{V}\left(\mathrm{t}, \mathrm{x}\left(\mathrm{t}, \mathrm{t}_{\mathrm{o}}, \varphi\right)\right)$ năo é diferenciável.

Estes pontos na verdade, são os valores inteiros onde a função retardamento da equação (1.3) dada por $r(t)=t-[t]$, é descontínua.

Teorema 3.4: Suponhamos que $u, v: \Re_{+} \rightarrow \Re_{+}$sejam funçőes contínuas, nåo decrescentes com $u(0)=v(0)=0, u(x)$ e $v(x)$ positivas para $x>0$.

Se existir uma funçăo $\mathrm{V}: \mathfrak{R} \times \mathfrak{R}^{\mathrm{n}} \rightarrow \mathfrak{R}$ definida positiva, dicotômica com relaçăo à equaçăo (1.3), satisfazendo 


$$
u(|x|) \leq V(t, x) \leq v(|x|), t \in \Re, x \in \Re^{n}
$$

entåo o equilíbrio nulo de (1.3) é estável.

Demonstraçăo: Seja $\Omega$ uma vizinhança da origem em $\mathrm{C}$ onde $\mathrm{V}$ é dicotômica com relaçăo à equaçăo (1.3). Para qualquer $\varepsilon>0$, existe um $\delta_{0}=\delta_{0}(\varepsilon), 0<\delta_{0}<\varepsilon$ tal que $v\left(\delta_{0}\right)<u(\varepsilon)$.

Tomemos $\varepsilon_{0}$ e $\delta_{0}$ suficientemente pequenos, $\delta_{0}<\varepsilon_{0}<\varepsilon \operatorname{com} v\left(\delta_{0}\right)<u\left(\varepsilon_{0}\right)$, tais que $\|\psi\|<\varepsilon_{0}$ implica que $\psi \in \Omega$.

Assim, dado $\mathrm{t}_{\mathrm{o}} \in \mathfrak{R}$, se $\|\varphi\|<\delta_{0}$ temos que :

$$
\mathrm{u}(|\varphi(\theta)|) \leq \mathrm{V}\left(\mathrm{t}_{\mathrm{o}}+\theta, \varphi(\theta)\right) \leq \mathrm{v}(|\varphi(\theta)|) \leq \mathrm{v}\left(\delta_{\mathrm{o}}\right)<\mathrm{u}\left(\varepsilon_{\mathrm{o}}\right),
$$

para $\theta \in[-1,0]$, isto é, $V\left(t, x\left(t, t_{0}, \varphi\right)\right)<u\left(\varepsilon_{0}\right)$ para $t \in\left[t_{0}-1, t_{0}\right]$ se $\|\varphi\|<\delta_{0}$.

Dado $\mu=\mu\left(t_{0}\right)$ pela Definição (1.13), a continuidade de $x\left(t, t_{0}, \varphi\right)$ com relação à $\varphi$, garante a existência de um $\delta_{1}=\delta_{1}\left(t_{0}, \varepsilon_{0}, \mu\right)>0, \delta_{1}<\varepsilon_{0}$ tal que a soluçăo $x\left(t, t_{0}, \varphi\right)$ existe em $I=\left[t_{0}-1, t_{0}+\mu\right]$ e $\left|x\left(t, t_{0}, \varphi\right)\right|<\varepsilon_{0}$ em I sempre que $\|\varphi\|<\delta_{1}$.

$E$ como $V$ é contínua e $V(t, 0)=0$, afirmamos que se $\|\varphi\|<\delta_{1}$ então $\mathrm{V}\left(\mathrm{t}, \mathrm{x}\left(\mathrm{t}, \mathrm{t}_{0}, \varphi\right)\right)<\mathrm{u}\left(\varepsilon_{0}\right)$ para $\mathrm{t} \in \mathrm{I}$.

Seja $\delta=\min \left\{\delta_{0}, \delta_{1}\right\}$. Mostremos que se $\|\varphi\|<\delta$ entăo $\mathrm{V}\left(\mathrm{t}, \mathrm{x}\left(\mathrm{t}, \mathrm{t}_{0}, \varphi\right)\right)<u\left(\varepsilon_{o}\right)$ para todo $\mathrm{t} \geq \mathrm{t}_{0}$.

Suponhamos então, por absurdo, que exista um primeiro instante năo inteiro $t_{1}>t_{0}+\mu$ tal que: 


$$
\begin{gathered}
\quad V\left(t_{1}, x\left(t_{1}, t_{0}, \varphi\right)\right)=u\left(\varepsilon_{0}\right) \\
\text { e } \quad v\left(t, x\left(t, t_{0}, \varphi\right)\right)<u\left(\varepsilon_{0}\right) \quad \text { para } t<t_{1} .
\end{gathered}
$$

Isto implica que $V^{\prime}\left(t_{1}, x_{t_{1}}\left(\cdot, t_{0}, \varphi\right)\right) \geq 0$, pois a funçăo $V\left(t, x\left(t, t_{0}, \varphi\right)\right)$ é diferenciável em $t_{1}$, desde que $t_{1}$ năo é inteiro.

Assim, como $\mathrm{V}$ é dicotômica com relaçăo à equaçăo (1.3), existe um $T=T\left(t_{0}, t_{1}\right), 0<T \leq t_{1}-t_{0}, \quad$ tal que :

$$
x_{t_{1}-T}\left(\cdot, t_{0}, \varphi\right) \in\left[\bar{\Omega}_{+}^{\prime}\left(t_{1}, T\right) \cup \Omega_{0}^{\prime}\left(t_{1}, T\right)\right] \subset \bigcup_{s} \Omega_{0 .}^{s}\left(t_{1}, T\right), \quad s \in(0, T] .
$$

Segue que $x_{t_{1}-T}\left(\cdot, t_{0}, \varphi\right) \in \Omega_{0-}^{s}\left(t_{1}, T\right)$, para algum $s \in(0, T]$, isto é, $\mathrm{V}\left(\mathrm{t}_{1}, \mathrm{x}\left(\mathrm{t}_{1}, \mathrm{t}_{1}-\mathrm{T}, \mathrm{x}_{\mathrm{t}_{1}-\mathrm{T}}\left(., \mathrm{t}_{0}, \varphi\right)\right)-\mathrm{V}\left(\mathrm{t}_{1}-\mathrm{s}, \mathrm{x}\left(\mathrm{t}_{1}-\mathrm{s}, \mathrm{t}_{1}-\mathrm{T}, \mathrm{x}_{\mathrm{t}_{1}-\mathrm{T}}\left(., \mathrm{t}_{0}, \varphi\right)\right) \leq 0\right.\right.$, ou seja, $\mathrm{V}\left(\mathrm{t}_{1}, \mathrm{x}\left(\mathrm{t}_{1}, \mathrm{t}_{0}, \varphi\right)\right) \leq \mathrm{V}\left(\mathrm{t}_{1}-\mathrm{s}, \mathrm{x}\left(\mathrm{t}_{1}-\mathrm{s}, \mathrm{t}_{0}, \varphi\right)\right)$, para algum $\mathrm{s} \in(0, \mathrm{~T}]$.

Assim, $\quad V\left(t_{1}, x\left(t_{1}, t_{0}, \varphi\right)\right)<u\left(\varepsilon_{0}\right)$, em contradição à nossa suposição inicial.E se $t_{1} \in Z$, ou $V^{\prime}\left(t_{1}\right) \geq 0$ ou $t_{1}$ é ponto de máximo local e pelo Lema (3.6) ou Definiçăo (1.13), temos também contradição.

Logo, se $\|\varphi\|<\delta$ temos que $V\left(t, x\left(t, t_{0}, \varphi\right)\right)<u\left(\varepsilon_{0}\right)$ para todo $t \geq t_{0}$.

Por hipótese, $u\left(\left|x\left(t, t_{0}, \varphi\right)\right|\right) \leq V\left(t, x\left(t, t_{0}, \varphi\right)\right)<u\left(\varepsilon_{0}\right)$ para $t \geq t_{0}$.

Como u é não decrescente, segue que $\left|x\left(t, t_{0}, \varphi\right)\right|<\varepsilon_{0}<\varepsilon$ para $t \geq t_{0}$. Portanto, o equilíbrio nulo de (1.3) é estável.

O próximo teorema prova a estabilidade assintótica do equilíbrio nulo da equaçăo (1.3), tendo como uma das hipóteses a existência de uma funçăo $\mathrm{V}$ estritamente dicotômica com relaçăo à equaçăo (1.3). 
Alguns lemas săo necessários para provar este resultado. Isto porque a funçăo $\mathrm{V}\left(\mathrm{t}, \mathrm{x}\left(\mathrm{t}, \mathrm{t}_{0}, \varphi\right)\right)$ năo é necessariamente diferenciável nos valores inteiros e também, se nestes valores $\mathrm{n}$ ocorrem máximos locais, nem sempre temos $V^{\prime}\left(n, x_{n}\left(\cdot, t_{c}, \varphi\right)\right)=0$. E assim, as propriedades de dicotomia $e$ dicotomia estrita nåo se aplicam.

Os Lemas (3.5) a (3.7) garantem estas propriedades para os casos citados, resultados estes fundamentais para a demonstraçăo do teorema.

Lema 3.5: Seja $\mathrm{f}: \mathfrak{R} \rightarrow \mathfrak{R}$ uma funçăo contínua, diferenciável em $\mathfrak{R}$, exceto possivelmente em um conjunto de medida nula, e seja c um ponto de máximo local de $f$.

Então, existe uma seqüência estritamente crescente $\left(x_{n}\right)_{n \in N}, x_{n} \rightarrow c$. quando $n \rightarrow \infty$, com $f^{\prime}\left(x_{n}\right) \geq 0$ para todo elemento $x_{n}$, onde $f^{\prime}(x)=\lim _{b \rightarrow c^{-}} \sup \frac{1}{h}[f(x+h)-f(x)]$.

Demonstração: Analisemos duas alternativas:

(i) existe uma vizinhança de c, onde o único ponto extremo de fé c, e

(ii) em toda vizinhança de $c$, existe pelo menos um ponto extremo de f.

Caso (i): Suponhamos que exista $\gamma>0$ tal que c seja o único ponto extremo de f em $(c-\gamma, c+\gamma)$; e que f seja não decrescente em $(c-\gamma, c)$ e não crescente em $(c, c+\gamma)$.

Assim, tomando a seqüéncia $\left(x_{n}\right)$ dada por $x_{n}=c-1 / n, n \in N$, existe $n_{0} \in N$ tal que para todo $n>n_{0}, x_{n} \in(c-\gamma, c)$. 
Logo, para a subseqüéncia $\left(x_{\mathrm{p}_{\mathrm{k}}}\right)$ onde $\mathrm{x}_{\mathrm{a}_{\mathrm{b}}}=\mathrm{x}_{\mathrm{b}_{\mathrm{p}}+\mathrm{k}}$, temos que $f^{\prime}\left(x_{n_{\mathfrak{k}}}\right) \geq 0$ para todo $x_{n_{\mathfrak{a}}}$, pois f é nåo decrescente em $(c-\gamma, c)$.

Caso (ii): Se o número de pontos extremos de f à esquerda de c for finito, o raciocínio é análogo ao caso (i). Analisemos o caso contrário, isto é, para todo $\gamma>0$ existe um ponto extremo de $f \mathrm{em}(\mathrm{c}-\gamma, \mathrm{c})$.

Assim, para $\gamma_{1}=1$ existe $x_{1} \in\left(c-\gamma_{1}, c\right)$ tal que $x_{1}$ é ponto extremo de f; para $\gamma_{2}=\min \left\{1 / 2,\left|c-x_{1}\right|\right\}$ existe $x_{2} \in\left(c-\gamma_{2}, c\right)$ tal que $x_{2}$ é ponto extremo de $f$.

Sucessivamente, para $\gamma_{\mathrm{n}}=\min \left\{1 / \mathrm{n},\left|\mathrm{c}-\mathrm{x}_{\mathrm{n}-1}\right|\right\}, \mathrm{n} \in \mathrm{N}$, existe $\mathrm{x}_{\mathrm{D}} \in\left(\mathrm{c}-\gamma_{\mathrm{n}}, \mathrm{c}\right)$ tal que $\mathrm{x}_{\mathrm{n}}$ é ponto extremo de $\mathrm{f}$.

Logo, temos uma seqüência estritamente crescente, por construçăo, $\left(x_{n}\right)_{n \in N}$ de pontos extremos de $f$ com $x_{n} \rightarrow c$ quando $n \rightarrow \infty$, e como fé contínua, $\left(\mathrm{x}_{\mathrm{n}}\right)_{\mathrm{n} \in \mathrm{N}}$ admite subseqüência $\left(\mathrm{x}_{\mathrm{n}_{\mathrm{k}}}\right)$ de pontos de mínimos locais de f e portanto $f^{\prime}\left(x_{n_{k}}\right) \geq 0$ para todo elemento $x_{\Sigma_{k}}$.

Assim, em ambos os casos temos o resultado desejado.

Lema 3.6: Seja $V: \Re \times \Re^{n} \rightarrow \Re$, dicotômica com relação à equação (1.3) (em $\Omega$ ). Se em algum instante inteiro $\bar{t}>t_{0}+\mu$, ocorrer um máximo local da funçăo $V\left(t, x\left(t, t_{0}, \varphi\right)\right)$, então $V\left(\bar{t}, x\left(\bar{t}, t_{0}, \varphi\right)\right) \leq V\left(\bar{t}-s, x\left(\bar{t}-s, t_{0}, \varphi\right)\right)$ para algum $s \in\left(0, \bar{t}-t_{0}\right]$, onde $\mu$ é como na Definição (1.13).

Demonstração: No caso em que $\mathrm{V}\left(\mathrm{t}, \mathrm{x}\left(\mathrm{t}, \mathrm{t}_{\mathrm{o}}, \varphi\right)\right)$ é constante em $(\overline{\mathrm{t}}-\gamma, \overline{\mathrm{t}})$ para algum $\gamma>0$, o resultado é imediato. Suponhamos então, que $V\left(t, x\left(t, t_{0}, \varphi\right)\right)$ seja estritamente crescente em $(\bar{t}-\gamma, \bar{t})$. 
Se $V\left(t, x\left(t, t_{0}, \varphi\right)\right)$ é diferenciável em $\bar{t}$, entăo $V^{\prime}\left(\bar{t}, x_{i}\left(\cdot, t_{0}, \varphi\right)\right)=0$. Assim por hipótese, existe $T=T\left(t_{0}, \bar{t}\right), 0<T \leq \bar{t}-t_{0}$, tal que:

$$
x_{i-T}\left(\cdot, t_{0}, \varphi\right) \in\left[\bar{\Omega}_{+}^{\prime}(\overline{\mathfrak{t}}, T) \cup \Omega_{0}^{\prime}(\overline{\mathfrak{t}}, T)\right] \subset \bigcup_{s} \Omega_{\mathrm{o} .}^{s}(\overline{\mathrm{t}}, T), \quad s \in(0, T]
$$

isto é, $V\left(\overline{\mathrm{t}}, \mathrm{x}\left(\overline{\mathrm{t}}, \mathrm{t}_{\mathrm{o}}, \varphi\right)\right) \leq \mathrm{V}\left(\overline{\mathrm{t}}-\mathrm{s}, \mathrm{x}\left(\overline{\mathrm{t}}-\mathrm{s}, \mathrm{t}_{\mathrm{o}}, \varphi\right)\right)$, para algum $\mathrm{s} \in(0, \mathrm{~T}]$ e temos o resultado.

Se $V\left(t, x\left(t, t_{0}, \varphi\right)\right)$ não é diferenciável em $\bar{t}$, pelo Lema (3.5) existe uma seqüência estritamente crescente $\left(t_{n}\right)_{n \in N}, t_{n} \rightarrow \bar{t}_{-}$quando $n \rightarrow \infty$ com $V^{\prime}\left(t_{n}, x_{t_{n}}\left(\cdot, t_{0}, \varphi\right)\right) \geq 0$ para todo $t_{n}, n \in N$.

Assim, como V é dicotômica com relação à equação (1.3) (em $\Omega$ ), para cada $t_{n} \geq t_{0}+\mu$, existe $T_{n}=T_{n}\left(t_{0}, t_{n}\right), 0<T_{n} \leq t_{n}-t_{0}$, tal que:

$\mathrm{x}_{\mathrm{t}_{\mathrm{n}}-\mathrm{T}_{\mathrm{n}}}\left(\cdot, \mathrm{t}_{0}, \varphi\right) \in\left[\bar{\Omega}_{+}^{\prime}\left(\mathrm{t}_{\mathrm{n}}, \mathrm{T}_{\mathrm{n}}\right) \cup \Omega_{0}^{\prime}\left(\mathrm{t}_{\mathrm{n}}, \mathrm{T}_{\mathrm{n}}\right)\right] \subset \Omega_{\mathrm{o}-}^{\mathrm{s}}\left(\mathrm{t}_{\mathrm{n}}, \mathrm{T}_{\mathrm{n}}\right)$, para algum $s \in\left(0, T_{n}\right]$, isto é,

$$
V\left(t_{n}, x\left(t_{n}, t_{0}, \varphi\right)\right) \leq V\left(t_{n}-s_{n}, x\left(t_{n}-s_{n}, t_{0}, \varphi\right)\right) \text {, para algum } s_{n} \in\left(0, T_{n}\right]
$$

Como $t_{n} \rightarrow \bar{t}_{\text {- quando }} \mathrm{n} \rightarrow \infty$ e $T_{n} \leq t_{n}-t_{0}$, temos que $\left(0, T_{n}\right] \subset\left(0, \bar{t}-t_{0}\right]$ e assim a seqüência $\left(s_{n}\right)_{n \in N}$ é limitada admitindo uma subseqüência $\left(s_{n_{k}}\right)$ convergente, digamos para $s, s \leq \bar{t}-t_{0}$. Logo,

$$
V\left(t_{n_{k}}, x\left(t_{n_{k}}, t_{0}, \varphi\right)\right) \leq V\left(t_{n_{k}}-s_{n_{k}}, x\left(t_{n_{k}}-s_{n_{k}}, t_{0}, \varphi\right)\right), s_{n_{k}} \in\left(0, T_{n_{k}}\right] \subset\left(0, \bar{t}-t_{o}\right] .
$$

Passando ao limite temos: $V\left(\overline{\mathrm{t}}, \mathbf{x}\left(\overline{\mathrm{t}}, \mathrm{t}_{0}, \varphi\right)\right) \leq \mathrm{V}\left(\overline{\mathrm{t}}-\mathrm{s}, \mathrm{x}\left(\overline{\mathrm{t}}-\mathrm{s}, \mathrm{t}_{\mathrm{o}}, \varphi\right)\right), \quad s \in\left(0, \overline{\mathrm{t}}-\mathrm{t}_{\mathrm{o}}\right]$.

Observemos que s é diferente de zero, pois como a funçăo $\mathrm{V}\left(\mathrm{t}, \mathrm{x}\left(\mathrm{t}, \mathrm{t}_{0}, \varphi\right)\right)$ é estritamente crescente em uma vizinhança à esquerda de $\overline{\mathrm{t}}$ 
e $V\left(t_{n}, x\left(t_{n}, t_{0}, \varphi\right)\right) \leq V\left(t_{n}-s_{n}, x\left(t_{n}-s_{n}, t_{0}, \varphi\right)\right)$ para todo $t_{n}, n \in N$, entáo os valores $\left(t_{n}-s_{n}\right)$ tendem a se afastar do valor $\bar{t}$, e assim $s$ tende a se afastar do zero.

Lema 3.7: Seja $V: \mathfrak{R} \times \mathfrak{R}^{\mathrm{n}} \rightarrow \mathfrak{R}$ estritamente dicotômica com relaçăo à equação (1.3). Se em algum instante inteiro $\overline{\mathrm{t}}>\mathrm{t}_{\mathrm{o}}+\mu$ ocorrer um máximo local da função $V\left(t, x\left(t, t_{o}, \varphi\right)\right)$, então existe pelo menos um $s \in(0, M]$ tal que:

$$
\mathrm{p}\left(\mathrm{V}\left(\overline{\mathrm{t}}, \mathrm{x}\left(\overline{\mathrm{t}}, \mathrm{t}_{\mathrm{o}}, \varphi\right)\right)\right) \leq \mathrm{V}\left(\overline{\mathrm{t}}-\mathrm{s}, \mathrm{x}\left(\overline{\mathrm{t}}-\mathrm{s}, \mathrm{t}_{\mathrm{o}}, \varphi\right)\right)
$$

onde $\mu, \mathrm{M}$ e p(t) são como na Definição (1.14).

Demonstração: Analogamente ao Lema (3.6), se $\mathrm{V}\left(\mathrm{t}, \mathrm{x}\left(\mathrm{t}, \mathrm{t}_{\mathrm{o}}, \varphi\right)\right)$ é diferenciável em $\overline{\mathrm{t}}$, temos a desigualdade estrita. Caso contrário, temos que: $\quad \mathrm{p}\left(\mathrm{V}\left(\mathrm{t}_{\mathrm{n}_{\mathbf{k}}}, \mathrm{x}\left(\mathrm{t}_{\mathrm{n}_{\mathbf{k}}}, \mathrm{t}_{\mathrm{o}}, \varphi\right)\right)\right)<\mathrm{V}\left(\mathrm{t}_{\mathrm{n}_{\mathbf{k}}}-\mathrm{s}_{\mathrm{n}_{\mathbf{k}}}, \mathrm{x}\left(\mathrm{t}_{\mathrm{n}_{\mathbf{k}}}-\mathrm{s}_{\mathrm{n}_{\mathbf{k}}}, \mathrm{t}_{\mathrm{o}}, \varphi\right)\right), \quad \mathrm{s}_{\mathrm{n}_{\mathbf{k}}} \in(0, \mathrm{M}]$, com $s_{n_{k}} \rightarrow s, \quad t_{n_{k}} \rightarrow \bar{t}_{-}$quando $n \rightarrow \infty$ e $s \in(0, M]$.

Passando ao limite temos $\mathrm{p}\left(\mathrm{V}\left(\overline{\mathrm{t}}, \mathrm{x}\left(\overline{\mathrm{t}}, \mathrm{t}_{0}, \varphi\right)\right)\right) \leq \mathrm{V}\left(\overline{\mathrm{t}}-\mathrm{s}, \mathrm{x}\left(\overline{\mathrm{t}}-\mathrm{s}, \mathrm{t}_{0}, \varphi\right)\right)$, para $s \in(0, M]$.

Lema 3.8: Sejam u,v e V como no Teorema (3.4). Além disso, suponhamos que $\mathrm{V}$ seja estritamente dicotômica com relação à equaçăo (1.3). Então sempre que para um determinado $t_{1}, t_{1}>t_{0}+\mu$ tivermos $\mathrm{V}\left(\mathrm{t}_{1}, \mathrm{x}\left(\mathrm{t}_{1}, \mathrm{t}_{\mathrm{o}}, \varphi\right)\right)>\mathrm{c}, \mathrm{c}>0$ e $\mathrm{t}_{1}$ é ponto de máximo local de $\mathrm{V}\left(\mathrm{t}, \mathrm{x}\left(\mathrm{t}, \mathrm{t}_{\mathrm{o}}, \varphi\right)\right)$, existirá um $\rho=\rho(c)>0$ tal que : 


$$
V\left(t_{1}, x\left(t_{1}, t_{0}, \varphi\right)\right)-V\left(t_{1}-s, x\left(t_{1}-s, t_{0}, \varphi\right)\right)<-\rho,
$$

para algum $s \in\left(0, T\left(t_{1}\right)\right]$, onde $\mu$ e $T\left(t_{1}\right)$ săo como na Definiçăo (1.14).

Demonstração: Pelo Teorema (3.4), existem $\delta_{1}>0$ e $\delta_{2}>0$ tais que se $\|\varphi\|<\delta_{1}$ então $V\left(t, x\left(t, t_{0}, \varphi\right)\right)<\delta_{2}$ para $t \geq t_{0}$.

Se $V\left(t_{1}, x\left(t_{1}, t_{0}, \varphi\right)\right)>c$, segue que $c<V\left(t_{1}, x\left(t_{1}, t_{0}, \varphi\right)\right)<\delta_{2}$.

Se $\mathrm{t}_{1}$ é ponto de máximo local de $\mathrm{V}\left(\mathrm{t}, \mathrm{x}\left(\mathrm{t}, \mathrm{t}_{0}, \varphi\right)\right)$, de acordo com $\mathrm{o}$ Lema (3.7) ou Definição (1.14), existe pelo menos um $s \in(0, M]$ tal que :

$$
\mathrm{p}\left(\mathrm{V}\left(\mathrm{t}_{1}, \mathrm{x}\left(\mathrm{t}_{1}, \mathrm{t}_{0}, \varphi\right)\right)\right) \leq \mathrm{V}\left(\mathrm{t}_{1}-\mathrm{s}, \mathrm{x}\left(\mathrm{t}_{1}-\mathrm{s}, \mathrm{t}_{\mathrm{o}}, \varphi\right)\right)
$$

onde $\mathrm{M}$ e $\mathrm{p}(\mathrm{t})$ são dados pela Definição de dicotomia estrita (1.14).

Seja $0<\rho(c)<\min \left\{p(y)-y: y \in\left[c, \delta_{2}\right]\right\}$. Assim, segue que:

$\mathrm{V}\left(\mathrm{t}_{1}-\mathrm{s}, \mathrm{x}\left(\mathrm{t}_{1}-\mathrm{s}, \mathrm{t}_{0}, \varphi\right)\right) \geq \mathrm{p}\left(\mathrm{V}\left(\mathrm{t}_{1}, \mathrm{x}\left(\mathrm{t}_{1}, \mathrm{t}_{0}, \varphi\right)\right)>\mathrm{V}\left(\mathrm{t}_{1}, \mathrm{x}\left(\mathrm{t}_{1}, \mathrm{t}_{0}, \varphi\right)\right)+\rho(\mathrm{c}) \quad\right.$ e portanto,

$\mathrm{V}\left(\mathrm{t}_{1}, \mathrm{x}\left(\mathrm{t}_{1}, \mathrm{t}_{0}, \varphi\right)\right)-\mathrm{V}\left(\mathrm{t}_{1}-\mathrm{s}, \mathrm{x}\left(\mathrm{t}_{1}-\mathrm{s}, \mathrm{t}_{0}, \varphi\right)\right)<-\rho(\mathrm{c})$, para algum $\mathrm{s} \in(0, \mathrm{M}]$.

Lema 3.9: Suponhamos $\mathrm{V}$ de classe $\mathrm{C}^{1}$ e estritamente dicotômica com relação à equação (1.3). Sejam $\mathrm{M}$ e $\mu$ as constantes dadas pela Definição (1.14). Então para cada $t_{1} \in R, \quad t_{1}>t_{0}+\mu$ em que $V^{\prime}\left(t_{1}, x_{t_{1}}\left(\cdot, t_{0}, \varphi\right)\right)>0$, existe um ponto $t_{2} \in\left(t_{1}, t_{1}+M\right)$ tal que $t_{2}$ é ponto de máximo local de $\mathrm{V}\left(\mathrm{t}, \mathrm{x}\left(\mathrm{t}, \mathrm{t}_{\mathrm{o}}, \varphi\right)\right)$. 
Demonstraçăo: Seja $t_{1} \in \Re, t_{1}>t_{0}+\mu \quad \operatorname{com~} V^{\prime}\left(t_{1}, x_{t_{1}}\left(\cdot, t_{0}, \varphi\right)\right)>0$. Suponhamos, por absurdo, que $V^{\prime}\left(t, x_{1}\left(\cdot, t_{0}, \varphi\right)\right) \geq 0$ para $t \in\left[t_{1}, t_{1}+M\right]$. De acordo com [19], considerando que $\mathrm{V}\left(\mathrm{t}, \mathrm{x}\left(\mathrm{t}, \mathrm{t}_{\mathrm{o}}, \varphi\right)\right)$ é contínua em $\left[t_{1}, t_{1}+M\right]$ e sua derivada (dada por limite superior à direita) é năo negativa em todo o intervalo, temos que:

$$
V\left(t_{1}+M, x\left(t_{1}+M, t_{0}, \varphi\right)\right) \geq V\left(t, x\left(t, t_{o}, \varphi\right)\right), \quad t \in\left[t_{1}, t_{1}+M\right]
$$

Assim,

$$
p\left(V\left(t_{1}+M, x\left(t_{1}+M, t_{0}, \varphi\right)\right)>V\left(t, x\left(t, t_{0}, \varphi\right)\right), \quad t \in\left[t_{1}, t_{1}+M\right]\right.
$$

Como $V^{\prime}\left(t_{1}+M, x_{t_{1}+M}\left(\cdot, t_{0}, \varphi\right)\right) \geq 0$, (3.3) contradiz o fato de $V$ ser estritamente dicotômica.

Observemos que se $V^{\prime}\left(t_{1}+M, x_{t_{1}+M}\left(\cdot, t_{0}, \varphi\right)\right)<0$ com $V^{\prime}\left(t, x_{t}\left(\cdot, t_{0}, \varphi\right)\right) \geq 0$ para todo $t \in\left[t_{1}, t_{1}+M\right)$, ou seja, se $\left(t_{1}+M\right)$ for um valor inteiro; então $\left(\mathrm{t}_{1}+\mathrm{M}\right)$ seria um ponto de máximo local de $\mathrm{V}\left(\mathrm{t}, \mathrm{x}\left(\mathrm{t}, \mathrm{t}_{\mathrm{o}}, \varphi\right)\right)$ com a validade de (3.2), e portanto de (3.3). Mas daí temos uma contradição do Lema (3.7).

Para simplificar, no próximo resultado usaremos a notação "x(t)" para $\left.x\left(t, t_{0}, \varphi\right)\right)$ e " $x_{t}$ " para $x_{t}\left(\cdot, t_{0}, \varphi\right)$.

Teorema 3.10: Suponhamos que $\mathrm{V}$ satisfaça todas as hipóteses do Teorema (3.4), e além disso que $\mathrm{V}$ seja de classe $\mathrm{C}^{1}$ e estritamente dicotômica com relação à equação (1.3) $(\mathrm{em} \Omega$ ). Então o equilíbrio nulo de (1.3) é assintoticamente estável. 
Demonstraçăo: Sejam $\delta$ e $u(\varepsilon)$ como no Teorema (3.4). Tomemos $\gamma \leq \delta$ e mostremos que se $\|\varphi\|<\gamma$, entăo $\left.x\left(t, t_{0}, \varphi\right)\right) \rightarrow 0$ quando $t \rightarrow \infty$.

Com relaçăo à $\left.x\left(t, t_{0}, \varphi\right)\right)$, pode ocorrer duas situaçŏes:

(i) existe um $\bar{t}>t_{0}+\mu$ tal que $V^{\prime}\left(t, x_{t}\right) \leq 0$ para todo $t \geq \bar{t}$, e

(ii) se năo ocorrer (i), o Lema (3.9) garante a existência de uma seqüência crescente $\left(m_{n}\right), m_{n} \rightarrow \infty$ quando $n \rightarrow \infty$, tal que $m_{n}$ é ponto de máximo local de $\mathrm{V}(\mathrm{t}, \mathrm{x}(\mathrm{t}))$.

caso (ii): Tomemos uma subseqüência de $\left(m_{n}\right)$, a seqüência $\left(t_{n}\right)$ dada por: $t_{1}=m_{1}, t_{2}$ é o primeiro elemento da seqüência $\left(m_{n}\right)$, digamos $m_{n_{0}}$, tal que $m_{n_{0}}-t_{1} \geq M$.

Assim definimos $t_{k}, k \geq 2$ como o primeiro elemento da seqüência $\left(m_{n}\right)$, digamos $m_{n_{j}}$, tal que $m_{n_{j}}-t_{k-1} \geq M$.

Como $\mathrm{m}_{\mathrm{n}} \rightarrow \infty$ quando $\mathrm{n} \rightarrow \infty$, é sempre possivel construir a seqüência $\left(t_{n}\right), n \in N$. Sejam

$$
\begin{aligned}
& c_{1}=\max \left\{V(t, x(t)): t_{0}+\mu \leq t \leq t_{1}\right\}, \\
& c_{j}=\max \left\{V(t, x(t)): t_{j-1} \leq t \leq t_{j}\right\}, j=2,3, \cdots e \\
& \ell_{j}=\min \left\{t \in\left[t_{j-1}, t_{j}\right]: c_{j}=V(t, x(t))\right\}, j=2,3, \cdots
\end{aligned}
$$

Provemos que a seqüência $\left(c_{j}\right)$ é estritamente decrescente.

Seja $\mathrm{c}_{\mathrm{j}}=\mathrm{V}\left(\ell_{\mathrm{j}}, \mathrm{x}\left(\ell_{\mathrm{j}}\right)\right)$ e $\ell_{\mathrm{j}}$ é ponto de máximo local de $\mathrm{V}(\mathrm{t}, \mathrm{x}(\mathrm{t}))$, $\mathrm{j}=2,3, \cdots$, entăo pelo Lema (3.7) ou pela Definiçăo (1.14), existe pelo menos um $s_{0} \in(0, M]$ tal que: 


$$
p\left(V\left(\ell, x\left(\ell_{j}\right)\right) \leq V\left(\ell,-s_{0}, x\left(\ell_{j}-s_{0}\right)\right), \quad j=2,3, \cdots\right.
$$

Por esta razăo, $\left(\ell_{j}-s_{0}\right)$ năo pertence à $\left[t_{j-1}, t_{j}\right]$ pois neste intervalo o máximo valor é $\mathrm{V}\left(\ell_{\mathrm{j}}, \mathrm{x}\left(\ell_{\mathrm{j}}\right)\right)$; e sim pertence ao intervalo $\left[\mathrm{t}_{\mathrm{j}-2}, \mathrm{t}_{\mathrm{j}-1}\right]$ uma vez que năo pode pertencer a intervalos anteriores considerando que a amplitude de cada um é, por construçăo, maior ou igual à $\mathrm{M}$.

$E$ no caso $\mathrm{j}=2$, temos que $\left(\ell_{2}-\mathrm{s}_{\mathrm{o}}\right)$ pertence ao intervalo $\left[\mathrm{t}_{\mathrm{o}}+\mu, \mathrm{t}_{1}\right]$ e assim, $V\left(\ell_{2}-s_{0}, x\left(\ell_{2}-s_{0}\right)\right) \leq c_{1}$.

Para $\mathrm{j}=2,3, \cdots$, temos $\mathrm{c}_{j}<\mathrm{p}\left(\mathrm{c}_{\mathrm{j}}\right) \leq \mathrm{V}\left(\ell_{\mathrm{j}}-\mathrm{s}_{\mathrm{o}}, \mathrm{x}\left(\ell_{\mathrm{j}}-\mathrm{s}_{\mathrm{o}}\right)\right) \leq \mathrm{c}_{\mathrm{j}-1}, \quad e$ assim, $c_{j}<c_{j-1}, \quad j=2,3, \cdots$

A seqüência $\left(c_{j}\right)$ sendo estritamente decrescente e limitada, é convergente e provemos então que $c_{j} \rightarrow 0$ quando $j \rightarrow \infty$. Suponhamos, por absurdo, que $\mathrm{c}_{\mathrm{j}} \rightarrow \mathrm{c}$ quando $\mathrm{j} \rightarrow \infty$. Então $\mathrm{V}\left(\ell_{\mathrm{j}}, \mathrm{x}\left(\ell_{\mathrm{j}}\right)\right)>\mathrm{c}$ e $\ell_{\mathrm{j}}$ é ponto de máximo local de $\mathrm{V}(\mathrm{t}, \mathrm{x}(\mathrm{t})), \mathrm{j}=2,3, \cdots$.

Pelo Lema (3.8), existe uma constante $\rho=\rho(c)>0$ tal que:

$$
\begin{gathered}
\mathrm{c}_{\mathrm{j}}<\mathrm{V}\left(\ell_{\mathrm{j}}-\mathrm{s}_{\mathrm{o}}, \mathrm{x}\left(\ell_{\mathrm{j}}-\mathrm{s}_{\mathrm{o}}\right)\right)-\rho \leq \mathrm{c}_{\mathrm{j}-1}-\rho, \quad \mathrm{j}=2,3, \cdots, \text { ou seja, } \\
\mathrm{c}_{\mathrm{j}+1}<\mathrm{c}_{\mathrm{j}}-\rho<\mathrm{c}_{\mathrm{j}-1}-2 \rho<\cdots<\mathrm{c}_{1}-\mathrm{j} \rho, \quad \mathrm{j} \in \mathrm{N} .
\end{gathered}
$$

Segue então que $c_{j} \rightarrow-\infty$ quando $j \rightarrow \infty$, o que contradiz a suposição inicial. Portanto $c=0$. $E \mathrm{u}(|\mathrm{x}(\mathrm{t})|) \leq \mathrm{V}(\mathrm{t}, \mathrm{x}(\mathrm{t}))$ com $\mathrm{V}(\mathrm{t}, \mathrm{x}(\mathrm{t})) \rightarrow 0$ quando $t \rightarrow \infty$. Como u é não decrescente e $u(0)=0$, segue que $x(t) \rightarrow 0$ quando $\mathrm{t} \rightarrow \infty$.

caso (i): Existe um $\overline{\mathrm{t}}>\mathrm{t}_{\mathrm{o}}+\mu$ tal que $\mathrm{V}^{\prime}\left(\mathrm{t}, \mathrm{x}_{\mathrm{t}}\right) \leq 0$ para $\mathrm{t} \geq \overline{\mathrm{t}}$.

Construímos a seqüência $\left(t_{n}\right)$ como anteriormente até obter um $n_{0}$ tal que $\mathrm{t}_{\mathrm{n}_{\mathrm{o}}}=\overline{\mathrm{t}}$ ou $\mathrm{t}_{\mathrm{n}_{\mathrm{o}}}+\mathrm{M} \geq \overline{\mathrm{t}}$. 
Pela hipótese do caso (i), segue que $V(t, x(t))$ é decrescente para $\mathrm{t} \geq \overline{\mathrm{t}}$.

Da limitaçăo de $V$, segue que $V(t, x(t)) \rightarrow c$ quanto $t \rightarrow \infty$. Assim: $\mathrm{V}(\mathrm{t}, \mathrm{x}(\mathrm{t}))-\mathrm{V}(\mathrm{t}-\mathrm{s}, \mathrm{x}(\mathrm{t}-\mathrm{s})) \rightarrow 0$ quando $\mathrm{t} \rightarrow \infty$, para todo $\mathrm{s} \in(0, \mathrm{M}], \mathrm{M}$ da definição de dicotomia estrita.

$$
\text { Logo, } x_{t-M} \rightarrow \bigcap_{s} \Omega_{0}^{s}(t, M) \text { quando } t \rightarrow \infty, s \in(0, M] \text {. }
$$

Se existir um $\tau>0$ tal que $V^{\prime}\left(t, x_{t}\right)<-\tau$ para $t \geq \bar{t}$, temos que, de acordo com [17], $V(t, x(t))$ é absolutamente contínua em [ $\bar{t}, t]$ para todo $t \geq \bar{t}$, e portanto vale:

$$
\mathrm{V}(\mathrm{t}, \mathrm{x}(\mathrm{t}))=\mathrm{V}(\overline{\mathrm{t}}, \mathrm{x}(\overline{\mathrm{t}}))+\int_{\mathrm{t}}^{\mathrm{t}} \mathrm{V}^{\prime}\left(\mathrm{u}, \mathrm{x}_{\mathrm{u}}\right) \mathrm{du}, \mathrm{e} \text { assim } \mathrm{V}(\mathrm{t}, \mathrm{x}(\mathrm{t})) \rightarrow-\infty \text { quando }
$$
$\mathrm{t} \rightarrow \infty$, o que contradiz o fato de $\mathrm{V}$ ser definida positiva. Se tal fato não ocorrer, dada a seqüência $(1 / n), n \in N$, existe uma seqüência $t_{n} \rightarrow \infty$ quando $n \rightarrow \infty$, tal que $V^{\prime}\left(t_{n}, x_{t_{n}}\right)>-1 / n$.

Logo, $x_{t_{n}-M} \rightarrow \Omega_{0}^{\prime}\left(t_{n}, M\right)$ quando $n \rightarrow \infty$.

Portanto, $x_{t_{n}-M} \rightarrow \bigcap_{s}\left[\Omega_{0}^{\prime}\left(t_{n}, M\right) \cap \Omega_{0}^{s}\left(t_{n}, M\right)\right]$ quando $n \rightarrow \infty$, para $s \in(0, M]$, e pelo ítem (iii) da Definição (1.14), segue que $x_{t_{n}} \rightarrow 0$ quando $\mathrm{n} \rightarrow \infty$.

Logo, $x\left(t_{n}\right) \rightarrow 0$ quando $n \rightarrow \infty$ e assim, $V\left(t_{n}, x\left(t_{n}\right)\right) \rightarrow 0$ quando $\mathrm{n} \rightarrow \infty$. Sendo $\mathrm{V}$ decrescente, segue que $\mathrm{V}(\mathrm{t}, \mathrm{x}(\mathrm{t})) \rightarrow 0$ quando $\mathrm{t} \rightarrow \infty$.

Como $u(|x(t)|) \leq V(t, x(t)), u(0)=0$ e $V(t, x(t)) \rightarrow 0$ quando $t \rightarrow \infty$, concluímos que $x(t) \rightarrow 0$ quando $t \rightarrow \infty$.

Portanto, em ambos os casos temos que o equilíbrio nulo de (1.3) é assintoticamente estável. 


\section{Capitulo 4}

\section{A RELAÇÃO ENTRE A EQUAÇÃO DIFERENCIAL E SUA EQUAÇÃO DISCRETA ASSOCIADA ATRAVÉS DE FUNÇÕES DICOTÓMICAS}

Neste capítulo fazemos uma comparação entre a aplicação do princípio de Dicotomia com relação à Equação Diferencial Geral (1.3) e com relação à Equação Discreta Associada (2.1).

Em alguns casos, a mesma função $\mathrm{V}$ pode ser utilizada para as duas equações. Por exemplo, com a função $V(x)=x^{2} / 2$, obtemos equivalência de resultados, trabalhando com a equação (2.5) (caso escalar), e com casos particulares da equação (1.3) (geral), que veremos através de exemplo.

\subsection{Caso Escalar da Equação Diferencial (2.5)}

A grande vantagem, neste caso, é que a equação restrita a intervalos $[n, n+1), \quad n \in Z$, se torna uma equação diferencial ordinária autônoma, onde a solução ou é constante ou estritamente monótona, de acordo com a Teoria Geral de Equações Diferenciais Ordinárias [12]. Formalmente temos o seguinte resultado:

Lema 4.1: As soluções não constantes de (2.5), no caso escalar, são seccionalmente estritamente monótonas, no seguinte sentido: em cada 
intervalo $\left[n_{0}+n, n_{0}+n+1\right], n \in Z_{+}$, a restriçăo $x_{n}(t)$ da soluçăo $x(t)$ de (2.5) é estritamente monótona.

Demonstraçăo: Inicialmente observemos que, conforme teoria do capítulo 2, $n_{0}$ é o primeiro inteiro maior ou igual ao instante inicial $t_{0}$.

Em cada intervalo $\left[n_{0}+n, n_{0}+n+1\right), n \in Z_{+}$, a solução $x(t)$ de (2.5) satisfaz:

$$
\begin{aligned}
& x_{n}^{\prime}(t)=f\left(x_{n}(t), c_{n}\right), \quad t \in\left[n_{o}+n, n_{o}+n+1\right) \\
& x_{n}\left(n_{0}+n\right)=c_{n}
\end{aligned}
$$

com $x_{n-1}\left(n_{o}+n\right)=c_{n}$, e pela unicidade de solução temos que os pontos críticos de $f\left(\cdot, c_{n}\right)$ são as soluções constantes de (4.1) e as demais são soluções estritamente monótonas, em virtude do sinal da função $f\left(\cdot, c_{n}\right)$.

O próximo lema contribui consideravelmente no estudo da estabilidade da solução nula da equação (2.5) no caso escalar, pois caracteriza completamente o comportamento da função $\mathrm{V}(\mathrm{x})=\mathrm{x}^{2} / 2$ ao longo das soluções de (2.5).

Lema 4.2: A função $V(x)=x^{2} / 2$ ao longo das soluções $x(t)$ de (2.5), caso escalar, apresenta máximos locais apenas nos valores inteiros para $\mathrm{t} \geq \mathrm{n}_{\mathrm{o}}$. Ou seja, para $\mathrm{n} \in \mathrm{Z}, \mathrm{n} \geq\left[\mathrm{t}_{\mathrm{o}}\right]+1=\mathrm{n}_{\mathrm{o}}$ :

$\max \left\{\mathrm{V}\left(\mathrm{x}\left(\mathrm{t}, \mathrm{t}_{\mathrm{o}}, \varphi\right)\right): \mathrm{t} \in[\mathrm{n}, \mathrm{n}+1]\right\}=\max \left\{\mathrm{V}\left(\mathrm{x}\left(\mathrm{n}, \mathrm{t}_{\mathrm{o}}, \varphi\right)\right), \mathrm{V}\left(\mathrm{x}\left(\mathrm{n}+1, \mathrm{t}_{\mathrm{o}}, \varphi\right)\right)\right\}$ 
Demonstraçăo: Este fato decorre diretamente do Lema (4.1), observando que $\mathrm{V}\left(\mathrm{x}\left(\mathrm{t}, \mathrm{t}_{\mathrm{o}}, \varphi\right)\right)$ pode apresentar minimo local no intervalo $(n, n+1)$ no instante em que, porventura, a soluçăo $x(t)$ se anule.

O próximo resultado mostra a relaçăo entre a equação (2.5), no caso escalar, e a sua equação discreta associada (2.1) através da teoria de Dicotomia.

Teorema 4.3: A função $\mathrm{V}(\mathrm{x})=\mathrm{x}^{2} / 2$ é dicotômica com relação à equação (2.5) (caso escalar), se e somente se, $\mathrm{V}$ é dicotômica com relação à equação discreta associada (2.1).

Demonstração: Suponhamos que $\mathrm{V}$ seja dicotômica com relação à equação (2.1) (em $\Omega=(-\delta, \delta) \subset \mathfrak{R}, \delta>0$ ). Provemos que, dado $\mathrm{t}_{\mathrm{o}} \in \mathfrak{R}$, existe um $\mu=\mu\left(t_{0}\right)>0$ tal que para todo $t \geq t_{0}+\mu$, existe em correspondência um $T=T\left(t_{0}, t\right), 0<T \leq t-t_{0}$, para o qual se verifica:

$$
\left[\bar{\Omega}_{+}^{\prime}(\mathrm{t}, \mathrm{T}) \cup \Omega_{\mathrm{o}}^{\prime}(\mathrm{t}, \mathrm{T})\right] \subset \bigcup_{\mathrm{s}} \Omega_{\mathrm{o}-}^{\mathrm{s}}(\mathrm{t}, \mathrm{T}), \quad \mathrm{s} \in(0, \mathrm{~T}],
$$

para alguma vizinhança $\Omega$ da origem em $C$, isto é:

sempre que $V^{\prime}\left(x_{t}\left(\cdot, t_{0}, \varphi\right) \geq 0\right.$, existe pelo menos um $s \in(0, T]$ tal que: $\mathrm{V}\left(\mathrm{x}\left(\mathrm{t}, \mathrm{t}_{\mathrm{o}}, \varphi\right)\right) \leq \mathrm{V}\left(\mathrm{x}\left(\mathrm{t}-\mathrm{s}, \mathrm{t}_{\mathrm{o}}, \varphi\right)\right)$.

Suponhamos então que $V^{\prime}\left(x_{t}\left(\cdot, t_{0}, \varphi\right) \geq 0\right.$ para algum $t \in[n-1, n)$, $n \in Z, \quad n \geq n_{o}+N\left(n_{o}\right)$, onde $N$ é dado pela Definição (1.6). Sabemos pelo Lema (4.2) que: 
$\max \left\{V\left(x\left(t, t_{0}, \varphi\right)\right): t \in[n-1, n]\right\}=\max \left\{V\left(x\left(n, t_{0}, \varphi\right)\right), V\left(x\left(n-1, t_{0}, \varphi\right)\right)\right\}$

Analisemos as duas possibilidades:

(i) o máximo valor é atingido no instante $(\mathrm{n}-1)$

Assim, $\mathrm{V}\left(\mathrm{x}\left(\mathrm{t}, \mathrm{t}_{\mathrm{o}}, \varphi\right)\right) \leq \mathrm{V}\left(\mathrm{x}\left(\mathrm{n}-1, \mathrm{t}_{\mathrm{o}}, \varphi\right)\right)$ e já temos um instante anterior à $\mathrm{t}$ com a propriedade desejada, desde que $t \neq n-1$. E 0 caso $t=n-1$ com $V^{\prime}\left(x_{t}\right) \geq 0$ só ocorre quando $V\left(x\left(t, t_{0}, \varphi\right)\right)$ é constante em $[n-1, n)$, e assim $\mathrm{V}(\mathrm{x}(\mathrm{n}-1))=\mathrm{V}(\mathrm{x}(\mathrm{n}))$ e segue pelo caso (ii).

(ii) o máximo valor é atingido no instante $\mathbf{n}$

Logo, $\mathrm{V}\left(\mathrm{x}\left(\mathrm{n}-1, \mathrm{t}_{\mathrm{o}}, \varphi\right)\right) \leq \mathrm{V}\left(\mathrm{x}\left(\mathrm{n}, \mathrm{t}_{\mathrm{o}}, \varphi\right)\right)$ ou, em linguagem de equação discreta, $V\left(x\left(n-1, n_{0}, c_{0}\right)\right) \leq V\left(x\left(n, n_{0}, c_{0}\right)\right)$ onde $n_{0}=\left[t_{0}\right]+1$ e $c_{0}=x\left(n_{0}, t_{0}, \varphi\right)$.

Assim, como $\mathrm{V}$ é dicotômica com relação à equação discreta associada (2.1) (em $\Omega=(-\delta, \delta)$ ), existe um inteiro não negativo $\mathrm{N}=\mathrm{N}\left(\mathrm{n}_{\mathrm{o}}\right)$, $\mathrm{N} \geq 2$; tal que para todo $\mathrm{n} \geq \mathrm{n}_{\mathrm{o}}+\mathrm{N}$, existe em correspondência um inteiro $\mathrm{k}=\mathrm{k}\left(\mathrm{n}_{\mathrm{o}}, \mathrm{n}\right), 2 \leq \mathrm{k} \leq \mathrm{n}-\mathrm{n}_{\mathrm{o}}$, para o qual:

$$
x\left(n-k, n_{o}, c_{o}\right) \in\left[\bar{\Omega}_{+}^{1}(n, k) \cup \Omega_{0}^{1}(n, k)\right] \subset \bigcup_{j=2}^{k} \Omega_{o-}^{j}(n, k) .
$$

Segue que $V\left(x\left(n, n-k, x\left(n-k, n_{0}, c_{0}\right)\right)-V\left(x\left(n-j, n-k, x\left(n-k, n_{0}, c_{0}\right)\right) \leq 0\right.\right.$, para algum $j, j=2, \cdots, k$, ou seja, $V\left(x\left(n, n_{o}, c_{0}\right)\right) \leq V\left(x\left(n-j, n_{o}, c_{o}\right)\right)$. $E$ como $V\left(x\left(t, t_{0}, \varphi\right)\right) \leq V\left(x\left(n, t_{0}, \varphi\right)\right)$, então $V\left(x\left(t, t_{0}, \varphi\right)\right) \leq V\left(x\left(n-j, t_{0}, \varphi\right)\right)$ para algum $j, j=2, \cdots, k$, e já temos um instante anterior à $t$ com a propriedade desejada. 
Seja $\mu\left(t_{0}\right)=N\left(n_{0}\right)=N\left(\left[t_{0}\right]+1\right)$, assim, para $t \geq t_{0}+\mu$ com $t \in[n-1, n)$, temos que $n \geq n_{0}+N\left(n_{0}\right)$, assumido no início da demonstraçăo.

Portanto, em ambos os casos temos que $\mathrm{V}$ é dicotómica com relaçăo à equaçăo (2.5) em $\Omega$, no caso escalar, com $\Omega=\{\varphi \in C:\|\varphi\|<\delta\}$, $\mu\left(t_{0}\right)=N\left(n_{o}\right)$ e para cada $t \geq t_{0}+\mu, T\left(t_{0}, t\right)=k\left(n, n_{0}\right)=k\left([t]+1,\left[t_{0}\right]+1\right)$.

Provemos agora a reciproca.

Seja V dicotômica com relação à equação (2.5), caso escalar, e provemos que $\mathrm{V}$ é dicotômica com relação à equação (2.1).

Suponhamos que $\mathrm{V}\left(\mathrm{x}\left(\mathrm{n}-1, \mathrm{n}_{\mathrm{o}}, \mathrm{c}_{\mathrm{o}}\right)\right) \leq \mathrm{V}\left(\mathrm{x}\left(\mathrm{n}, \mathrm{n}_{\mathrm{o}}, \mathrm{c}_{\mathrm{o}}\right)\right)$, para algum instante $n, n>t_{0}+\mu+1$.

Como o máximo de $\mathrm{V}\left(\mathrm{x}\left(\mathrm{t}, \mathrm{t}_{\mathrm{o}}, \varphi\right)\right)$ em $[\mathrm{n}-1, \mathrm{n}]$ ocorre nos valores extremos, temos dois casos a considerar:

(i) $V^{\prime}\left(x_{n}\left(\cdot, t_{0}, \varphi\right) \geq 0 \quad e\right.$

(ii) $\mathrm{V}\left(\mathrm{x}\left(\mathrm{t}, \mathrm{t}_{0}, \varphi\right)\right)$ assume máximo local no instante $\mathrm{n}$.

Caso (i): $V^{\prime}\left(x_{n}\left(\cdot, t_{0}, \varphi\right) \geq 0\right.$. Pela hipótese, existe um $\mu=\mu\left(t_{0}\right)>0$ e uma vizinhança $\Omega$ da origem em $C$, tal que, para $n \geq t_{0}+\mu$, existe em correspondência um $T=T\left(t_{0}, n\right), \quad 0<T \leq n-t_{0}$, para o qual:

$$
\mathrm{x}_{\mathrm{n}-\mathrm{T}}\left(\cdot, \mathrm{t}_{\mathrm{o}}, \varphi\right) \in\left[\bar{\Omega}_{+}^{\prime}(\mathrm{n}, \mathrm{T}) \dot{\cup} \Omega_{\mathrm{o}}^{\prime}(\mathrm{n}, \mathrm{T})\right] \subset \bigcup_{\mathrm{s}} \Omega_{\mathrm{o}-}^{\mathrm{s}}(\mathrm{n}, \mathrm{T}), \quad \mathrm{s} \in(0, \mathrm{~T}]
$$

Segue que $\left.x_{n-T}\left(\cdot, t_{0}, \varphi\right)\right) \in \Omega_{0}^{s}(n, T)$, para algum $s \in(0, T]$. Assim, $\mathrm{V}\left(\mathrm{x}\left(\mathrm{n}, \mathrm{t}_{0}, \varphi\right)\right) \leq \mathrm{V}\left(\mathrm{x}\left(\mathrm{n}-\mathrm{s}, \mathrm{t}_{0}, \varphi\right)\right)$, para algum $\mathrm{s} \in(0, \mathrm{~T}]$.

Analisemos as seguintes situações: 
(i-1) $V\left(x\left(n-1, t_{0}, \varphi\right)\right)<V\left(x\left(n, t_{0}, \varphi\right)\right)$

(i-2) $V\left(x\left(n-1, t_{0}, \varphi\right)\right)=V\left(x\left(n, t_{0}, \varphi\right)\right)$.

Caso (i-1): $V\left(x\left(n-1, t_{0}, \varphi\right)\right)<V\left(x\left(n, t_{0}, \varphi\right)\right)$

Como $V\left(x\left(n, t_{0}, \varphi\right)\right) \leq V\left(x\left(n-s, t_{0}, \varphi\right)\right)$, para algum $s \in(0, T]$, pelo Lema (4.2), obrigatoriamente $n-s<n-1$, onde $(n-s) \in[n-(j+1), n-j)$, para algum $\mathrm{j} \in \mathrm{Z}_{+}$.

Se $j=1$, ou seja, $(n-s) \in[n-2, n-1)$, obrigatoriamente o máximo valor de $\mathrm{V}\left(\mathrm{x}\left(\mathrm{t}, \mathrm{t}_{\mathrm{o}}, \varphi\right)\right)$ neste intervalo é $\mathrm{V}\left(\mathrm{x}\left(\mathrm{n}-2, \mathrm{t}_{\mathrm{o}}, \varphi\right)\right)$, pelas considerações acima.

Portanto, $\mathrm{V}\left(\mathrm{x}\left(\mathrm{n}, \mathrm{t}_{0}, \varphi\right)\right) \leq \mathrm{V}\left(\mathrm{x}\left(\mathrm{n}-\mathrm{s}, \mathrm{t}_{0}, \varphi\right)\right) \leq \mathrm{V}\left(\mathrm{x}\left(\mathrm{n}-2, \mathrm{t}_{0}, \varphi\right)\right), \quad$ ou seja, para $\mathrm{j}=1$ temos que $\mathrm{V}\left(\mathrm{x}\left(\mathrm{n}, \mathrm{n}_{\mathrm{o}}, \mathrm{c}_{0}\right)\right) \leq \mathrm{V}\left(\mathrm{x}\left(\mathrm{n}-2, \mathrm{n}_{\mathrm{o}}, \mathrm{c}_{\mathrm{o}}\right)\right)$, e nos demais casos, $\mathrm{V}\left(\mathrm{x}\left(\mathrm{n}, \mathrm{t}_{\mathrm{o}}, \varphi\right)\right) \leq \mathrm{V}\left(\mathrm{x}\left(\mathrm{n}-\mathrm{s}, \mathrm{t}_{\mathrm{o}}, \varphi\right)\right) \leq \mathrm{V}\left(\mathrm{x}\left(\mathrm{m}, \mathrm{t}_{\mathrm{o}}, \varphi\right)\right)$, onde $\mathrm{V}\left(\mathrm{x}\left(\mathrm{m}, \mathrm{t}_{\mathrm{o}}, \varphi\right)\right)$ é o máximo valor da função em $[n-(j+1), n-j)$, com $j \neq 1$.

Portanto, no caso ( $\mathrm{i}-1)$ temos que:

$$
\mathrm{V}\left(\mathrm{x}\left(\mathrm{n}, \mathrm{n}_{\mathrm{o}}, \mathrm{c}_{\mathrm{o}}\right)\right) \leq \mathrm{V}\left(\mathrm{x}\left(\mathrm{n}-\mathrm{j}, \mathrm{n}_{\mathrm{o}}, \mathrm{c}_{\mathrm{o}}\right)\right)
$$

para algum $j \in Z_{+}, j \geq 2$, que é o resultado desejado.

Caso (i-2): $\mathrm{V}\left(\mathrm{x}\left(\mathrm{n}-1, \mathrm{t}_{\mathrm{o}}, \varphi\right)\right)=\mathrm{V}\left(\mathrm{x}\left(\mathrm{n}, \mathrm{t}_{\mathrm{o}}, \varphi\right)\right)$

Temos que $V\left(x\left(n, t_{0}, \varphi\right)\right) \leq V\left(x\left(n-s, t_{0}, \varphi\right)\right)$, para algum $s \in(0, T]$. Se $n-s<n-1$, segue que $V\left(x\left(n, t_{0}, \varphi\right)\right) \leq V\left(x\left(n-s, t_{0}, \varphi\right)\right) \leq V\left(x\left(n-j, t_{0}, \varphi\right)\right)$ para algum $j \in Z_{+}, j \geq 2$, onde $V\left(x\left(n-j, t_{0}, \varphi\right)\right)$ é o máximo valor de $\mathrm{V}\left(\mathrm{x}\left(\mathrm{t}, \mathrm{t}_{\mathrm{o}}, \varphi\right)\right)$ no intervalo que contém $\mathrm{o}$ instante $(\mathrm{n}-\mathrm{s})$.

Se $n-s=n-1$, analisemos o intervalo $[n-2, n-1]$. 
Se $V\left(x\left(n-2, t_{0}, \varphi\right)\right) \geq V\left(x\left(n-1, t_{0}, \varphi\right)\right)=V\left(x\left(n, t_{0}, \varphi\right)\right)$, temos 0 resultado desejado. Caso contrário, pelo Lema (4.2) concluímos que o instante $(\mathrm{n}-1)$ é ponto de máximo local da funçăo $\mathrm{V}\left(\mathrm{x}\left(\mathrm{t}, \mathrm{t}_{\mathrm{o}}, \varphi\right)\right)$ e pelo Lema (3.6), segue que:

$\mathrm{V}\left(\mathrm{x}\left(\mathrm{n}-1, \mathrm{t}_{0}, \varphi\right)\right) \leq \mathrm{V}\left(\mathrm{x}\left(\mathrm{n}-1-\tau, \mathrm{t}_{0}, \varphi\right)\right)$, para algum $\tau \in\left(0, \mathrm{n}-1-\mathrm{t}_{\mathrm{o}}\right]$, desde que $n-1>t_{0}+\mu$. Logo,

$$
V\left(x\left(n, t_{0}, \varphi\right)\right) \leq V\left(x\left(n-1-\tau, t_{0}, \varphi\right)\right) \leq V\left(x\left(n-j, t_{0}, \varphi\right)\right)
$$

para algum $j \in Z_{+}, j \geq 2$, e temos o resultado desejado, concluíndo o caso (i).

Caso (ii): $\mathrm{V}\left(\mathrm{x}\left(\mathrm{t}, \mathrm{t}_{\mathrm{o}}, \varphi\right)\right)$ assume máximo valor no instante $\mathrm{n}$

Assim, pelo Lema (3.6) temos que $V\left(x\left(n, t_{0}, \varphi\right)\right) \leq V\left(x\left(n-s, t_{0}, \varphi\right)\right)$, para algum $s \in\left(0, n-t_{0}\right.$ ] e segue análogo ao caso (i).

Portanto, em ambos os casos temos que $\mathrm{V}$ é dicotômica com relação à equaçăo (2.1) (em $\Omega$ ), caso escalar, com $\mathrm{N}\left(\mathrm{n}_{\mathrm{o}}\right)=\left[\mu\left(\mathrm{t}_{\mathrm{o}}\right)\right]+2 \geq 2$ e $\Omega=(-\delta, \delta)$, onde $\delta$ é o raio da vizinhança $\Omega$ da origem em C.

Observemos que $\mathrm{N}$ foi tomado de tal forma a garantir que, para $\mathrm{n} \geq \mathrm{n}_{\mathrm{o}}+\mathrm{N}\left(\mathrm{n}_{\mathrm{o}}\right)$ temos $\mathrm{n}-1>\mathrm{t}_{\mathrm{o}}+\mu$ (assumido no início da demonstraçăo).

\section{2: Caso Geral (Não Escalar) da Equação Diferencial (1.3)}

Dada uma funçăo $\mathrm{V}$, dicotômica com relaçăo à equaçăo (1.3), nem sempre a mesma funçăo será dicotômica com relaçăo à equaçăo discreta 
associada, pois neste caso, năo necessariamente temos a validade da propriedade dada pelo Lema (4.2).

Sendo assim, definimos uma nova funçăo a partir de $\mathrm{V}$, denotada por $\overline{\mathrm{V}}$, atuando ao longo das soluçóes de (2.1) que terá a propriedade de dicotomia desejada. A saber:

$$
\bar{V}\left(n, x\left(n, n_{o}, c_{0}\right)\right)=\operatorname{máx}\left\{V\left(t, x\left(t, t_{0}, \varphi\right)\right): t \in[n, n+1]\right\}, n \in Z, n \geq n_{0} \text {, }
$$

e nos intervalos $(n, n+1), n \in Z$, define-se $\bar{V}\left(t, x\left(t, t_{0}, \varphi\right)\right)$ como sendo 0 segmento de reta entre os valores $\bar{V}\left(n, x\left(n, n_{0}, c_{0}\right)\right)$ e $\bar{V}\left(n+1, x\left(n+1, n_{0}, c_{0}\right)\right)$.

Nestas condições, provemos o seguinte resultado:

Teorema 4.4: Seja $V: \Re \times \Re^{\mathrm{n}} \rightarrow \mathfrak{R}$ uma função dicotômica com relaçăo à equação (1.3) (em $\Omega$ ). Então, a função $\overline{\mathrm{V}}$ definida em (4.2), é dicotômica com relação à equação discreta associada (2.1).

Demonstração: Com a hipótese dada, provemos que existe uma vizinhança $\Omega$ da origem em $\Re^{\mathrm{n}}$ e um $\mathrm{N}=\mathrm{N}\left(\mathrm{n}_{\mathrm{o}}\right), \mathrm{N} \in \mathrm{Z}_{+}, \mathrm{N} \geq 2$, tal que para todo $n \geq n_{0}+N$, existe em correspondência um $k=k\left(n_{0}, n\right)$, $k \in Z_{+}, 2 \leq k \leq n-n_{0}$, com a seguinte propriedade:

sempre que $\overline{\mathrm{V}}\left(\mathrm{n}-1, \mathrm{x}\left(\mathrm{n}-1, \mathrm{n}_{\mathrm{o}}, \mathrm{c}_{\mathrm{o}}\right)\right) \leq \overline{\mathrm{V}}\left(\mathrm{n}, \mathrm{x}\left(\mathrm{n}, \mathrm{n}_{\mathrm{o}}, \mathrm{c}_{\mathrm{o}}\right)\right)$, entăo para algum $j, j=2, \cdots, k$, temos $\bar{V}\left(n, x\left(n, n_{0}, c_{0}\right)\right) \leq \bar{V}\left(n-j, x\left(n-j, n_{o}, c_{0}\right)\right)$.

Para simplificar a notaçăo, denotemos $x\left(t, t_{0}, \varphi\right)$ por " $x(t) ", x_{t}\left(\cdot, t_{0}, \varphi\right)$ por " $x_{t}$ " e analogamente $x\left(n, n_{o}, c_{0}\right)$ por " $x(n)$ ". Seja

$$
\bar{t}_{\mathrm{n}}=\min \{t \in[\mathrm{n}, \mathrm{n}+1]: \mathrm{V}(\mathrm{t}, \mathrm{x}(\mathrm{t}))=\overline{\mathrm{V}}(\mathrm{n}, \mathrm{x}(\mathrm{n}))\}, \mathrm{n} \geq \mathrm{n}_{\mathrm{o}}
$$


Suponhamos entăo que $\bar{V}(n-1, x(n-1)) \leq \bar{V}(n, x(n))$ para algum índice n. Analisemos as seguintes situaçőes:

(i) $\bar{t}_{n}$ é ponto de máximo local de $V(t, x(t))$

(ii) o caso contrário ao ítem (i).

Caso (i): $\bar{t}_{\mathrm{n}}$ é ponto de máximo local de $\mathrm{V}(\mathrm{t}, \mathrm{x}(\mathrm{t})$ )

Neste caso temos duas alternativas:

(i-1) $\bar{t}_{n}$ é um inteiro

(i-2) o caso contrário ao ítem (i-1).

Caso (i-1): $\bar{t}_{n}$ é um inteiro

Então, pelo Lema (3.6) segue que $\mathrm{V}\left(\overline{\mathrm{t}}_{\mathrm{n}}, \mathrm{x}\left(\overline{\mathfrak{t}}_{\mathrm{n}}\right)\right) \leq \mathrm{V}\left(\overline{\mathrm{t}}_{\mathrm{n}}-\overline{\mathrm{s}}, \mathrm{x}\left(\overline{\mathrm{t}}_{\mathrm{n}}-\overline{\mathrm{s}}\right)\right)$ para algum $\bar{s} \in\left(0, \bar{t}_{\mathrm{n}}-\mathrm{t}_{\mathrm{o}}\right]$.

Temos que $\left(\overline{\mathrm{t}}_{\mathrm{n}}-\overline{\mathrm{s}}\right) \in[\mathrm{n}-\mathrm{k}, \mathrm{n}-\mathrm{k}+1]$, para algum $\mathrm{k} \in \mathrm{Z}_{+}$, onde $\mathrm{k} \neq 0$ pois $\left(\overline{\mathfrak{t}}_{\mathrm{n}}-\overline{\mathrm{s}}\right)$ não pode pertencer ao intervalo $[\mathrm{n}, \mathrm{n}+1]$, considerando que $\overline{\mathrm{t}}_{\mathrm{n}}-\overline{\mathrm{s}}_{\mathbf{\mathrm { t }}} \overline{\mathrm{t}}_{\mathrm{n}}$ e $\bar{t}_{n}$ é o mínimo valor neste intervalo onde a função $V(t, x(t))$ assume seu máximo valor. Logo,

$$
\overline{\mathrm{V}}(\mathrm{n}, \mathrm{x}(\mathrm{n}))=\mathrm{V}\left(\overline{\mathrm{t}}_{\mathrm{n}}, \mathrm{x}\left(\overline{\mathrm{t}}_{\mathrm{n}}\right)\right) \leq \mathrm{V}\left(\overline{\mathrm{t}}_{\mathrm{n}}-\overline{\mathrm{s}}, \mathrm{x}\left(\overline{\mathrm{t}}_{\mathrm{n}}-\overline{\mathrm{s}}\right)\right) \leq \overline{\mathrm{V}}(\mathrm{n}-\mathrm{k}, \mathrm{x}(\mathrm{n}-\mathrm{k})),
$$

para algum $\mathrm{k} \geq 1, \mathrm{k} \in \mathrm{Z}_{+}$.

Se $k>1$, já temos o instante anterior à $n$ com a propriedade desejada.

Se $k=1,\left(\overline{\mathfrak{t}}_{\mathrm{n}}-\overline{\mathrm{s}}\right) \in[\mathrm{n}-1, \mathrm{n})$.

Neste caso, obrigatoriamente $\overline{\mathrm{V}}(\mathrm{n}, \mathrm{x}(\mathrm{n}))=\overline{\mathrm{V}}(\mathrm{n}-1, \mathrm{x}(\mathrm{n}-1))=\mathrm{V}\left(\overline{\mathrm{t}}_{\mathrm{n}}-\overline{\mathrm{s}}, \mathrm{x}\left(\overline{\mathrm{t}}_{\mathrm{n}}-\overline{\mathrm{s}}\right)\right)$, desde que $\overline{\mathrm{V}}(\mathrm{n}-1, \mathrm{x}(\mathrm{n}-1)) \leq \overline{\mathrm{V}}(\mathrm{n}, \mathrm{x}(\mathrm{n}))$. 
Analisemos o ponto $\overline{\mathrm{t}}_{\mathrm{n}-1}$. As alternativas såo:

(i-1-1) $\bar{t}_{n-1}$ é ponto de máximo local de $V(t, x(t))$ e

(i-1-2) o caso contrário ao ítem (i-1-1).

Caso (i-1-1):

Se $\bar{t}_{n-1}$ é ponto de máximo local de $\mathrm{V}(\mathrm{t}, \mathrm{x}(\mathrm{t}))$, pelo Lema (3.6) ou pela Definição (1.13) temos que $V\left(\bar{t}_{n-1}, x\left(\bar{t}_{n-1}\right)\right) \leq V\left(\bar{t}_{n-1}-s, x\left(\bar{t}_{n-1}-s\right)\right)$, para algum $s \in\left(0, \bar{t}_{n-1}-t_{0}\right]$. Logo,

$$
\begin{gathered}
\bar{V}(n, x(n))=\bar{V}(n-1, x(n-1))=V\left(\bar{t}_{n-1}, x\left(\bar{t}_{n-1}\right)\right) \leq \\
\leq V\left(\bar{t}_{n-1}-s, x\left(\bar{t}_{n-1}-s\right)\right) \leq \bar{V}(n-j, x(n-j)),
\end{gathered}
$$

onde $\left(\overline{\mathfrak{t}}_{n-1}-s\right) \in[n-j, n-j+1]$, para algum $j \in Z_{+}, j \geq 2$.

Observemos que o Lema (3.6) só é válido se $\overline{\mathrm{t}}_{\mathrm{n}-1}>\mathrm{t}_{\mathrm{o}}+\mu$. Para tanto, tomemos $\mathrm{N}\left(\mathrm{n}_{\mathrm{o}}\right)=\left[\mu\left(\mathrm{t}_{\mathrm{o}}\right)\right]+2$, pois para $\mathrm{n} \geq \mathrm{n}_{\mathrm{o}}+\mathrm{N}$, temos que:

$$
\overline{\mathrm{t}}_{\mathrm{n}-1} \geq \mathrm{n}-1 \geq \mathrm{n}_{\mathrm{o}}+\mathrm{N}-1=\left[\mathrm{t}_{\mathrm{o}}\right]+\left[\mu\left(\mathrm{t}_{\mathrm{o}}\right)\right]+2>\mathrm{t}_{\mathrm{o}}+\mu \text {. }
$$

Caso (i-1-2): $\overline{\mathrm{t}}_{\mathrm{n}-1}$ năo é ponto de máximo local de $\mathrm{V}(\mathrm{t}, \mathrm{x}(\mathrm{t}))$

Obrigatoriamente, $\bar{t}_{n-1}=n-1$ pois os casos $\bar{t}_{n-1}=n$ e $\bar{t}_{n-1}$, ponto interior ao intervalo $[\mathrm{n}-1, \mathrm{n}]$ não ocorrem, considerando que $\overline{\mathrm{V}}(\mathrm{n}, \mathrm{x}(\mathrm{n}))=\overline{\mathrm{V}}(\mathrm{n}-1, \mathrm{x}(\mathrm{n}-1))$ e a hipótese deste sub-caso. E como $\overline{\mathfrak{t}}_{\mathrm{n}-1}=\mathrm{n}-1$ năo é ponto de máximo local, então existe pelo menos um $\mathrm{t}_{1}$, $\mathrm{n}-2 \leq \mathrm{t}_{1}<\mathrm{n}-1$ tal que $\mathrm{V}\left(\mathrm{t}_{1}, \mathrm{x}\left(\mathrm{t}_{1}\right)\right)>\mathrm{V}(\mathrm{n}-1, \mathrm{x}(\mathrm{n}-1))$. Assim,

$$
\begin{aligned}
\overline{\mathrm{V}}(\mathrm{n}-2, \mathrm{x}(\mathrm{n}-2)) & =\operatorname{máx}\{\mathrm{V}(\mathrm{t}, \mathrm{x}(\mathrm{t})): \mathrm{t} \in[\mathrm{n}-2, \mathrm{n}-1]\} \geq \mathrm{V}\left(\mathrm{t}_{1}, \mathrm{x}\left(\mathrm{t}_{1}\right)\right)> \\
& >\mathrm{V}(\mathrm{n}-1, \mathrm{x}(\mathrm{n}-1))=\mathrm{V}\left(\overline{\mathrm{t}}_{\mathrm{n}-1}, \mathrm{x}\left(\overline{\mathrm{t}}_{\mathrm{n}-1}\right)\right)=\overline{\mathrm{V}}(\mathrm{n}-1, \mathrm{x}(\mathrm{n}-1))
\end{aligned}
$$


Logo, $\quad \bar{V}(n, x(n))=\bar{V}(n-1, x(n-1))=V\left(\bar{t}_{n-1}, x\left(\bar{t}_{n-1}\right)\right)<\bar{V}(n-2, x(n-2)), \quad e$ temos o resultado desejado, concluindo o caso (i-1).

Caso (i-2): $\overline{\mathrm{t}}_{\mathrm{n}}$ năo é um inteiro

Neste caso $V^{\prime}\left(\bar{t}_{n}, x_{\mathfrak{i}_{n}}\right)=0$ e como $\mathrm{V}$ é dicotómica com relaçăo à equaçăo (1.3), existe um $T=T\left(t_{0}, \bar{t}_{n}\right), 0<T \leq \bar{t}_{n}-t_{0}$, tal que:

$$
V\left(\overline{\mathfrak{t}}_{n}, x\left(\overline{\mathrm{t}}_{\mathrm{n}}\right)\right) \leq \mathrm{V}\left(\overline{\mathrm{t}}_{\mathrm{n}}-\mathrm{s}, \mathrm{x}\left(\overline{\mathrm{t}}_{\mathrm{n}}-\mathrm{s}\right)\right)
$$

para algum $s \in(0, T]$, e segue análogo ao caso (i-1).

Caso (ii): $\bar{t}_{\mathrm{n}}$ não é ponto de máximo local de $\mathrm{V}(\mathrm{t}, \mathrm{x}(\mathrm{t}))$

Então $\bar{t}_{n}=n+1$ pois se $\bar{t}_{n}$ é ponto interior de $[n, n+1]$ ou se $\bar{t}_{n}=n$ com $\overline{\mathrm{V}}(\mathrm{n}, \mathrm{x}(\mathrm{n})) \geq \overline{\mathrm{V}}(\mathrm{n}-1, \mathrm{x}(\mathrm{n}-1))$, teríamos um valor máximo de $\mathrm{V}(\mathrm{t}, \mathrm{x}(\mathrm{t}))$ ocorrendo em $\bar{t}_{n}$.

Logo, como $\bar{t}_{\mathrm{n}}$ é o mínimo valor de $[\mathrm{n}, \mathrm{n}+1]$ onde $\mathrm{V}(\mathrm{t}, \mathrm{x}(\mathrm{t}))$ assume seu máximo valor neste intervalo, $\overline{\mathrm{t}}_{\mathrm{n}}=\mathrm{n}+1$ e $\overline{\mathrm{t}}_{\mathrm{n}}$ não é ponto de máximo local de $V(t, x(t))$, afirmamos que existe pelo menos um $t_{1}>\bar{t}_{n} \operatorname{com} V^{\prime}\left(t_{1}, x_{t_{1}}\right) \geq 0$ e $V\left(t_{1}, x\left(t_{1}\right)\right)>V\left(\bar{t}_{n}, x\left(\bar{t}_{n}\right)\right)$.

Como V é dicotômica com relação à equação (1.3), existe um $\mathrm{T}=\mathrm{T}\left(\mathrm{t}_{\mathrm{o}}, \mathrm{t}_{1}\right), 0<\mathrm{T} \leq \mathrm{t}_{1}-\mathrm{t}_{\mathrm{o}}$, tal que $\mathrm{V}\left(\mathrm{t}_{1}, \mathrm{x}\left(\mathrm{t}_{1}\right)\right) \leq \mathrm{V}\left(\mathrm{t}_{1}-\mathrm{s}, \mathrm{x}\left(\mathrm{t}_{1}-\mathrm{s}\right)\right)$ para algum $s \in(0, T]$ com $t_{1} \geq t_{0}+\mu$, considerando $N\left(n_{0}\right)=\left[\mu\left(t_{0}\right)\right]+2$. Logo,

$$
\begin{gathered}
\bar{V}(n, x(n))=V\left(\bar{t}_{n}, x\left(\bar{t}_{n}\right)\right)<V\left(t_{1}, x\left(t_{1}\right)\right) \leq \\
\leq V\left(t_{1}-s, x\left(t_{1}-s\right)\right) \leq \bar{V}(n-k, x(n-k)),
\end{gathered}
$$


para algum $k \in Z_{+}, k \geq 2$, considerando que $\left(t_{1}-s\right) \in[n-k, n-k+1)$. Observemos que $t_{1}-s<n-1$, pois $\bar{V}(n, x(n))=V\left(\bar{t}_{n}, x\left(\bar{t}_{n}\right)\right)<V\left(t_{1}, x\left(t_{1}\right)\right)$ com $\overline{\mathrm{V}}(\mathrm{n}, \mathrm{x}(\mathrm{n})) \geq \overline{\mathrm{V}}(\mathrm{n}-1, \mathrm{x}(\mathrm{n}-1))$.

Portanto, em ambos os casos analisados temos que $\overline{\mathrm{V}}$ é dicotómica com relaçăo à equaçăo discreta (2.1) (em $\Omega$ ), com $\Omega=\left\{x \in \mathfrak{R}^{n}:|x|<\delta\right\}$ e $\mathrm{N}\left(\mathrm{n}_{\mathrm{o}}\right)=\left[\mu\left(\mathrm{t}_{\mathrm{o}}\right)\right]+2$, onde $\delta$ é o raio da vizinhança $\Omega$ da origem em $\mathrm{C}$, garantida pela Definição de dicotomia (1.13).

Teorema 4.5: Seja $\mathrm{V}: \mathfrak{R} \times \mathfrak{R}^{\mathrm{n}} \rightarrow \mathfrak{R}$ uma funçăo estritamente dicotômica com relação à equação (1.3) (em $\Omega$ ), satisfazendo todas as hipóteses do Teorema (3.10). Então, a função $\overline{\mathrm{V}}$ definida em (4.2) é estritamente dicotômica com relação à equação (2.1).

Demonstração: Suponhamos que para algum indice $n$, temos $\overline{\mathrm{V}}(\mathrm{n}-1, \mathrm{x}(\mathrm{n}-1)) \leq \overline{\mathrm{V}}(\mathrm{n}, \mathrm{x}(\mathrm{n}))$.

Caso (i): $\overline{\mathrm{t}}_{\mathrm{n}}$ é ponto de máximo local de $\mathrm{V}(\mathrm{t}, \mathrm{x}(\mathrm{t})$ )

Analisemos, neste caso, duas alternativas:

(i-1) $\bar{t}_{\mathrm{n}}$ é um inteiro

(i-2) o caso contrário ao ítem (i-1).

Caso ( $\mathrm{i}-1)$ : $\overline{\mathrm{t}}_{\mathrm{n}}$ é um inteiro

Então, pelo Lema (3.7) existe pelo menos um $s \in(0, M]$ tal que:

$$
\mathrm{V}\left(\overline{\mathrm{t}}_{\mathrm{n}}, \mathrm{x}\left(\overline{\mathrm{t}}_{\mathrm{n}}\right)<\mathrm{p}\left(\mathrm{V}\left(\overline{\mathrm{t}}_{\mathrm{n}}, \mathrm{x}\left(\overline{\mathrm{t}}_{\mathrm{n}}\right)\right) \leq \mathrm{V}\left(\overline{\mathrm{t}}_{\mathrm{n}}-\mathrm{s}, \mathrm{x}\left(\overline{\mathrm{t}}_{\mathrm{n}}-\mathrm{s}\right)\right)\right.\right.
$$


onde $\mathrm{M}$ e $\mathrm{p}(\mathrm{t})$ săo dados na Definiçăo de dicotomia estrita (1.14).

Logo, se $\left(\bar{t}_{n}-s\right) \in[n-k, n-k+1] \subset\left[\bar{t}_{n}-M, \bar{t}_{n}\right)$ para algum $k \in Z_{+}, k \geq 2$, temos que $\bar{V}(n, x(n))<p\left(\bar{V}(n, x(n)) \leq V\left(\bar{t}_{n}-s, x\left(\bar{t}_{n}-s\right)\right) \leq \bar{V}(n-k, x(n-k))\right.$.

Observemos que $k \neq 1$, pois:

$$
\overline{\mathrm{V}}(\mathrm{n}-1, \mathrm{x}(\mathrm{n}-1)) \leq \overline{\mathrm{V}}(\mathrm{n}, \mathrm{x}(\mathrm{n}))=\mathrm{V}\left(\overline{\mathrm{t}}_{\mathrm{n}}, \mathrm{x}\left(\overline{\mathrm{t}}_{\mathrm{n}}\right)\right)<\mathrm{V}\left(\overline{\mathrm{t}}_{\mathrm{n}}-\mathrm{s}, \mathrm{x}\left(\overline{\mathrm{t}}_{\mathrm{n}}-\mathrm{s}\right)\right),
$$

ou seja, $V\left(\bar{t}_{n}-s, x\left(\bar{t}_{n}-s\right)\right.$ ) é maior que o máximo valor de $V(t, x(t))$ no intervalo $[\mathrm{n}-1, \mathrm{n}]$.

Analisemos as duas alternativas:

(i-1-1) $\overline{\mathrm{t}}_{\mathrm{n}-\mathrm{k}}$ é ponto de máximo local de $\mathrm{V}(\mathrm{t}, \mathrm{x}(\mathrm{t}))$

(i-1-2) o caso contrário ao ítem (i-1-1).

Caso (i-1-1): $\overline{\mathfrak{t}}_{\mathrm{n}-\mathrm{k}}$ é ponto de máximo local

Então, pelo Lema (3.7) ou pela Definição (1.14), existe pelo menos um $s \in(0, M]$ tal que $p\left(V\left(\bar{t}_{n-k}, x\left(\bar{t}_{n-k}\right)\right) \leq V\left(\bar{t}_{n-k}-s, x\left(\bar{t}_{n-k}-s\right)\right)\right.$. Assim,

$$
\overline{\mathrm{V}}(\mathrm{n}-\mathrm{k}, \mathrm{x}(\mathrm{n}-\mathrm{k}))=\mathrm{V}\left(\overline{\mathrm{t}}_{\mathrm{n}-\mathrm{k}}, \mathrm{x}\left(\overline{\mathrm{t}}_{\mathrm{n}-\mathrm{k}}\right)\right)<\mathrm{p}\left(\mathrm{V}\left(\overline{\mathrm{t}}_{\mathrm{n}-\mathrm{k}}, \mathrm{x}\left(\overline{\mathrm{t}}_{\mathrm{n}-\mathrm{k}}\right)\right) \leq \overline{\mathrm{V}}(\mathrm{n}-\tau, \mathrm{x}(\mathrm{n}-\tau)),\right.
$$

para algum $\tau \in Z, \tau \geq 3$, onde $\left(\overline{\mathrm{t}}_{\mathrm{n}-\mathrm{k}}-\mathrm{s}\right) \in[\mathrm{n}-\tau, n-\tau+1]$ com $\tau>k$. Logo, para algum $t \in Z, t \geq 3$, temos:

$$
\mathrm{p}(\overline{\mathrm{V}}(\mathrm{n}, \mathrm{x}(\mathrm{n})) \leq \overline{\mathrm{V}}(\mathrm{n}-\mathrm{k}, \mathrm{x}(\mathrm{n}-\mathrm{k}))<\overline{\mathrm{V}}(\mathrm{n}-\tau, \mathrm{x}(\mathrm{n}-\tau)) .
$$

Observemos que o Lema (3.7) só é válido se $\bar{t}_{n-k}>t_{0}+\mu$, onde $2 \leq k \leq M$, pois $\bar{t}_{n-k} \in[n-k, n-k+1] \subset\left[\bar{t}_{n}-M, \bar{t}_{n}\right) \subset[n-M, n+1)$. 
Para tanto, tomemos $N\left(n_{0}\right)=\left[\mu\left(t_{0}\right)\right]+M+1$, pois para $n \geq n_{0}+N\left(n_{0}\right)$, temos que $\bar{t}_{\mathrm{n}-\mathrm{k}} \geq \mathrm{n}-\mathrm{k} \geq \mathrm{n}_{\mathrm{o}}+\left[\mu\left(\mathrm{t}_{\mathrm{o}}\right)\right]+1>\mathrm{t}_{\mathrm{o}}+\mu$.

Caso (i-1-2): $\overline{\mathfrak{t}}_{\mathrm{n}-\mathrm{k}}$ năo é ponto de máximo local

Entăo, necessariamente $\overline{\mathrm{t}}_{\mathrm{n}-\mathrm{k}}$ ocorre nos extremos do intervalo $[\mathrm{n}-\mathrm{k}, \mathrm{n}-\mathrm{k}+1]$.

No caso em que $\bar{t}_{n-k}=n-k$, existe pelo menos um $t, n-k-1 \leq t<n-k$ com $V(t, x(t))>V\left(\bar{t}_{n-k}, x\left(\bar{t}_{n-k}\right)\right)$.

Logo, $\overline{\mathrm{V}}(\mathrm{n}-\mathrm{k}, \mathrm{x}(\mathrm{n}-\mathrm{k}))<\mathrm{V}(\mathrm{t}, \mathrm{x}(\mathrm{t})) \leq \overline{\mathrm{V}}(\mathrm{n}-\mathrm{k}-1, \mathrm{x}(\mathrm{n}-\mathrm{k}-1))$ e segue que:

$$
\mathrm{p}(\overline{\mathrm{V}}(\mathrm{n}, \mathrm{x}(\mathrm{n})) \leq \overline{\mathrm{V}}(\mathrm{n}-\mathrm{k}, \mathrm{x}(\mathrm{n}-\mathrm{k}))<\overline{\mathrm{V}}(\mathrm{n}-\mathrm{k}-1, \mathrm{x}(\mathrm{n}-\mathrm{k}-1)), \mathrm{k} \in \mathrm{Z}, \mathrm{k} \geq 2 .
$$

Se $\overline{\mathrm{t}}_{\mathrm{n}-\mathrm{k}}=\mathrm{n}-\mathrm{k}+1$ e considerando que $\overline{\mathrm{t}}_{\mathrm{n}-\mathrm{k}}$ não é ponto de máximo local, existe pelo menos um $t>\bar{t}_{n-k}$ com $V(t, x(t))>V\left(\bar{t}_{n-k}, x\left(\bar{t}_{n-k}\right)\right) \quad e$ $V^{\prime}\left(t, x_{t}\right) \geq 0$.

Como $\mathrm{V}$ é estritamente dicotômica com relação à equação (1.3), existe um $\mathrm{T}=\mathrm{T}\left(\mathrm{t}_{\mathrm{o}}, \mathrm{t}\right), 0<\mathrm{T} \leq \mathrm{t}-\mathrm{t}_{\mathrm{o}}$, tal que $\mathrm{p}(\mathrm{V}(\mathrm{t}, \mathrm{x}(\mathrm{t}))<\mathrm{V}(\mathrm{t}-\mathrm{s}, \mathrm{x}(\mathrm{t}-\mathrm{s}))$, para algum $s \in(0, T]$, assim,

$$
\begin{aligned}
\mathrm{p}(\overline{\mathrm{V}}(\mathrm{n}, \mathrm{x}(\mathrm{n})) & \leq \overline{\mathrm{V}}(\mathrm{n}-\mathrm{k}, \mathrm{x}(\mathrm{n}-\mathrm{k}))<\mathrm{V}(\mathrm{t}, \mathrm{x}(\mathrm{t}))< \\
& <\mathrm{p}(\mathrm{V}(\mathrm{t}, \mathrm{x}(\mathrm{t}))<\overline{\mathrm{V}}(\mathrm{n}-\tau, \mathrm{x}(\mathrm{n}-\tau)),
\end{aligned}
$$

para algum $\tau \in Z, \tau \geq 3$ com $(t-s) \in[n-\tau, n-\tau+1]$ e concluímos o caso (i-1).

Caso (i-2): $\bar{t}_{\mathrm{n}}$ năo é um inteiro 
Neste caso $V^{\prime}\left(\bar{t}_{n}, x_{t_{n}}\right) \geq 0$ e como $V$ é estritamente dicotómica com relaçăo à equaçăo (1.3), existe um $T=T\left(t_{0}, \bar{t}_{n}\right), 0<T \leq \bar{t}_{n}-t_{n}$, tal que: $p\left(V\left(\bar{t}_{n}, x\left(\bar{t}_{n}\right)\right)<V\left(\bar{t}_{n}-s, x\left(\bar{t}_{n}-s\right)\right)\right.$, para algum $s \in(0, T]$, isto é,

$$
\mathrm{p}(\overline{\mathrm{V}}(\mathrm{n}, \mathrm{x}(\mathrm{n}))<\overline{\mathrm{V}}(\mathrm{n}-\mathrm{k}, \mathrm{x}(\mathrm{n}-\mathrm{k}))
$$

para algum $k \in Z, k \geq 2$ com $\left(\bar{t}_{n}-s\right) \in[n-k, n-k+1]$.

Observemos que $k \neq 1$, pois $\bar{V}(n, x(n)) \geq \bar{V}(n-1, x(n-1))$ e concluímos o caso (i).

Caso (ii): $\bar{t}_{\mathrm{n}}$ não é ponto de máximo local de $\mathrm{V}(\mathrm{t}, \mathrm{x}(\mathrm{t}))$

Então $\bar{t}_{n}=n+1$, pois se $\bar{t}_{n}$ é ponto interior de $[n, n+1]$ ou se $\bar{t}_{n}=n$ com $\overline{\mathrm{V}}(\mathrm{n}, \mathrm{x}(\mathrm{n})) \geq \overline{\mathrm{V}}(\mathrm{n}-1, \mathrm{x}(\mathrm{n}-1))$, teríamos um valor máximo de $\mathrm{V}(\mathrm{t}, \mathrm{x}(\mathrm{t}))$ ocorrendo em $\overline{\mathfrak{t}}_{\mathrm{n}}$. E mais, $V^{\prime}\left(\overline{\mathfrak{t}}_{\mathrm{n}}, \mathrm{x}_{\mathfrak{i}_{n}}\right) \geq 0$ e segue análogo ao caso anterior (i-2).

Concluíndo parcialmente, em ambos os casos temos provado os ítens (i) e (ii) da Definição (1.7).

Para completar a demonstração, provemos o ítem (iii) da definição.

Seja $x_{n-k}\left(c_{o}\right) \rightarrow \bigcap_{j=1}^{k} \Omega_{o}^{j}(n, k)$ quando $n \rightarrow \infty$. Provemos que $x_{n}\left(c_{o}\right) \rightarrow 0$ quando $\mathrm{n} \rightarrow \infty$.

Como $c_{j}=\operatorname{máx}\left\{\bar{V}(n, x(n)): n \in\left[t_{j-1}, t_{j}\right], n \in Z\right\}$ conforme notação do Teorema (3.10) e definição de $\overline{\mathrm{V}}$, temos que $c_{j} \rightarrow c$ quando $j \rightarrow \infty$ para alguma constante c, e pelo mesmo Teorema segue que, necessariamente $c=0$ e que $x(t) \rightarrow 0$ quando $t \rightarrow \infty$.

Logo, $x_{n}\left(c_{0}\right) \rightarrow 0$ quando $n \rightarrow \infty$ e temos a validade do ítem (iii) da Definição (1.7). 
Concluindo, temos que $\overline{\mathrm{V}}$ é estritamente dicotômica com relaçăo à equaçăo (2.1) (em $\Omega$ ), com $\Omega=\left\{x \in \mathfrak{R}^{\mathrm{n}}:|x|<\delta\right\}, N\left(n_{0}\right)=\left[\mu\left(t_{0}\right)\right]+M+1$ e $\mathrm{k}\left(\mathrm{n}, \mathrm{n}_{\mathrm{o}}\right)=2 \mathrm{M}$ para todo $\mathrm{n} \geq \mathrm{n}_{\mathrm{o}}+\mathrm{N}$, onde $\delta$ é o raio da vizinhança $\Omega$ da origem em C, garantida pela Definiçăo (1.13).

No parágrafo (4.1), utilizando a funçăo $\mathrm{V}(\mathrm{x})=\mathrm{x}^{2} / 2$, vimos que 0 conceito de dicotomia (dicotomia estrita) para a equaçăo (2.5) e para a equação discreta associada, no caso escalar, é equivalente.

No parágrafo (4.2) comentamos que, em geral, não temos a equivalência para a equaçăo (1.3). Porém, observemos que, para casos particulares apresentando a propriedade dada pelo Lema (4.2), podemos ter o resultado. Vejamos um exemplo.

Exemplo 4.1: Consideremos o sistema:

$$
\begin{aligned}
& x^{\prime}(t)=-x(t) x([t])-y(t) \sqrt{x^{2}(t)+y^{2}(t)} \text { q.t.p., } t \geq 0 \\
& y^{\prime}(t)=-y(t) x([t])+x(t) \sqrt{x^{2}(t)+y^{2}(t)} \text { q.t.p., } t \geq 0
\end{aligned}
$$

Em coordenadas polares, em cada intervalo $[n, n+1), n \in Z$, o sistema equivalente é:

$$
\begin{array}{ll}
r^{\prime}(t)=r(t) r_{n} \cos \theta_{n} & r(n)=r_{n} \\
\theta^{\prime}(t)=r(t) & \theta(n)=\theta_{n},
\end{array}
$$

que tem como soluçăo, para $t \in[n, n+1), n \in Z$, 


$$
\begin{aligned}
& r(t)=r_{n} \exp \left[r_{n} \cos \theta_{n}(n-t)\right] \\
& \theta(t)=\left[1-\exp \left(r_{n} \cos \theta_{n}(n-t)\right)\right] / \cos \theta_{n}+\theta_{n},
\end{aligned}
$$

E a equaçăo discreta associada é dada por:

$$
\begin{aligned}
& r_{n+1}=r_{n} \exp \left(-r_{n} \cos \theta_{n}\right) \\
& \theta_{n+1}=\left[1-\exp \left(-r_{n} \cos \theta_{n}\right)\right] / \cos \theta_{n}+\theta_{n} .
\end{aligned}
$$

Através de dados computacionais, trabalhando com a função $\mathrm{V}(\mathrm{x}, \mathrm{y})=\left(\mathrm{x}^{2}+\mathrm{y}^{2}\right) / 2$ (ou $\left.\mathrm{V}(\mathrm{r})=\mathrm{r}^{2} / 2\right)$, constatamos que o processo de dicotomia se faz presente, bastando analisá-lo através da equação discreta, uma vez que $\mathrm{V}$ ao longo das soluções de (4.3) assume valores extremos apenas nos instantes inteiros.

De fato:

Para $\mathrm{t} \in[\mathrm{n}, \mathrm{n}+1), \mathrm{n} \in \mathrm{Z}$ e $\mathrm{z}\left(\mathrm{t}, \mathrm{t}_{\mathrm{o}}, \varphi\right)$ denotando a solução de (4.3) em coordenadas polares, temos que:

$$
V^{\prime}\left(z_{t}\left(\cdot, t_{0}, \varphi\right)=r^{\prime}(t) r(t)=-r^{2}(t) r_{n} \cos \theta_{n}\right.
$$

E $V^{\prime}\left(z_{t}\left(\cdot, t_{0}, \varphi\right)\right)=0$, se e somente se, ocorrer uma das seguintes alternativas: $r_{n}=0, \cos \theta_{n}=0$ ou $r(t)=0$ para $t \in[n, n+1)$.

Analisando os três casos constatamos que a única alternativa é $r(t)$ ser uma função constante em $[n, n+1)$.

De fato, considerando que $r(t)=r_{n} \exp \left[r_{n} \cos \theta_{n}(n-t)\right], t \in[n, n+1)$, temos:

(i) Se $r_{n}=0, r(t)=0$ para todo $t \in[n, n+1)$,

(ii) Se $\cos \theta_{n}=0, r(t)=r_{n}$ para todo $t \in[n, n+1)$, 
(iii) Se $r(t)=0$ para algum $t \in[n, n+1)$, necessariamente $r_{n}=0$ e segue análogo ao caso (i).

Logo, para as funçőes $r(t)$ năo constantes, $V\left(z\left(t, t_{0}, \varphi\right)\right)$ năo apresenta pontos extremos interiores aos intervalos $[n, n+1), n \in Z$; e temos a propriedade do Lema (4.2). 


\section{Capitulo 5}

\section{APLICAÇŌES DA TEORIA DE FUNÇŌES DICOTÓMICAS}

Neste capítulo apresentamos algumas aplicações da Teoria de Funçōes Dicotômicas evidenciando, no caso escalar, a forte influência que a equaçăo discreta exerce sobre a equação diferencial funcional a que está associada. E para simplificar, usaremos "x(t)" para denotar a solução $x\left(t, t_{0}, \varphi\right)$; e " $x_{t}$ " para $x_{t}\left(\cdot, t_{0}, \varphi\right)$.

Aplicação 1: Consideremos a equação

$$
x^{\prime}(t)=a x(t)+b x([t]) \text { q.t.p., } t \geq 0
$$

com $a \leq-\delta<0$ e $|\mathrm{b}|<\mathrm{k} \delta, \delta>0$ e algum $\mathrm{k} \in(0,1)$.

Nestas condições, o equilíbrio nulo de (5.1) é assintoticamente estável.

Primeiramente observemos que se $b>0$, entăo $-a / b>1 / k$ e se $\mathrm{b}<0, \mathrm{a} / \mathrm{b}>1 / \mathrm{k}$.

Seja $V(x)=x^{2} / 2$. Provemos que $V$ é estritamente dicotômica com relaçăo à equaçăo (5.1) e pelo Teorema (3.10) tomando $u(x)=x^{2} / 4$ e $v(x)=x^{2}$ para $x>0$, temos o resultado desejado. 
Temos que $V^{\prime}\left(x_{1}\right)=x(t) x^{\prime}(t)=x(t)[a x(t)+b x([t])]$.

Inicialmente observemos que para $t \in Z_{+} \operatorname{com} x(t) \neq 0, V^{\prime}\left(x_{1}\right)<0$.

De fato:

Como $x(t)=x([t])$ e $(a+b)<0$, entăo $V^{\prime}\left(x_{1}\right)=x^{2}(t)(a+b)<0$.

Portanto, analisemos os pontos $t \in R_{+} \backslash Z, x(t) \neq 0 \operatorname{com} V^{\prime}\left(x_{1}\right) \geq 0$, isto é,

$$
x(t)[a x(t)+b x([t])] \geq 0 .
$$

Analisemos dois casos: $\mathrm{x}(\mathrm{t})>0$ e $\mathrm{x}(\mathrm{t})<0$.

1) $x(t)>0$.

Então, $a x(t)+b x([t]) \geq 0$.

Se $b>0$, segue que $x([t]) \geq(-a / b) x(t)>x(t) / k$.

Assim, $x^{2}([t]) / 2>x^{2}(t) / 2 k^{2}$ e escolhendo a função $p(y)=y / k^{2}, y>0$, temos $\mathrm{p}(\mathrm{V}(\mathrm{x}(\mathrm{t})))<\mathrm{V}(\mathrm{x}([\mathrm{t}]))$.

Se $b<0$, segue que $x([t]) \leq(-a / b) x(t)<-x(t) / k$.

Considerando que $x(t)>0$, temos:

$$
\begin{gathered}
\mathrm{x}^{2}([\mathrm{t}]) / 2>\mathrm{x}^{2}(\mathrm{t}) / 2 \mathrm{k}^{2}, \text { ou seja, } \\
\mathrm{p}(\mathrm{V}(\mathrm{x}(\mathrm{t})))<\mathrm{V}(\mathrm{x}([\mathrm{t}]))
\end{gathered}
$$

2) $x(t)<0$

Devemos ter $a x(t)+b x([t]) \leq 0$.

Se $b>0, x([t]) \leq(a / b)(-x(t))<(-1 / k)(-x(t))=x(t) / k \leq 0$, assim,

$$
\begin{gathered}
\mathrm{x}^{2}([\mathrm{t}]) / 2>\mathrm{x}^{2}(\mathrm{t}) / 2 \mathrm{k}^{2}, \quad \text { ou seja, } \\
\mathrm{p}(\mathrm{V}(\mathrm{x}(\mathrm{t})))<\mathrm{V}(\mathrm{x}([\mathrm{t}]))
\end{gathered}
$$


Se $b<0, x([t]) \geq(-a / b) x(t)>-x(t) / k$, e assim,

$$
\begin{gathered}
\mathrm{x}^{2}([\mathrm{t}]) / 2>\mathrm{x}^{2}(\mathrm{t}) / 2 \mathrm{k}^{2}, \text { ou seja, } \\
\mathrm{p}(\mathrm{V}(\mathrm{x}(\mathrm{t})))<\mathrm{V}(\mathrm{x}([\mathrm{t}]))
\end{gathered}
$$

Logo, em ambos os casos, sempre que $V^{\prime}\left(x_{t}\right) \geq 0$ e $x(t) \neq 0$ segue que $\mathrm{p}(\mathrm{V}(\mathrm{x}(\mathrm{t})))-\mathrm{V}(\mathrm{x}([\mathrm{t}]))<0$ para $\mathrm{t} \in \mathfrak{R}_{+} \backslash \mathrm{Z}$.

Observemos o caso $x(t)=0$ com $V^{\prime}\left(x_{t}\right)=0$. Consideremos:

(i) $t \in \mathfrak{R}_{+} \backslash Z$

(ii) $t \in Z_{+}$

caso (i): Analisemos duas situações com $t \in(n, n+1), n \in Z_{+}$:

(i-1) existe pelo menos um $\overline{\mathrm{t}} \in(\mathrm{n}, \mathrm{n}+1)$ com $\mathrm{x}(\overline{\mathrm{t}}) \neq 0$ e $\mathrm{V}^{\prime}\left(\mathrm{x}_{\mathrm{t}}\right) \geq 0$,

$(\mathrm{i}-2) \mathrm{x}(\mathrm{t})=0$ para todo $\mathrm{t} \in(\mathrm{n}, \mathrm{n}+1)$.

caso (i-1): Neste caso, $\mathrm{p}(\mathrm{V}(\mathrm{x}(\overline{\mathrm{t}})))<\mathrm{V}(\mathrm{x}(\mathrm{n}))$, como já vimos.

Logo, $0=\mathrm{p}(\mathrm{V}(\mathrm{x}(\mathrm{t})))<\mathrm{p}(\mathrm{V}(\mathrm{x}(\overline{\mathrm{t}})))<\mathrm{V}(\mathrm{x}(\mathrm{n}))$ e já temos o resultado desejado $\operatorname{com} \mathrm{T}(\mathrm{t})=1$.

caso (i-2): Neste caso, necessariamente temos que $x(t)=0$ para todo $\mathrm{t} \geq \mathrm{n}$, pela propriedade de dicotomia já provada. Assim, como $x(t)$ é solução não nula, existe pelo menos um $\overline{\mathrm{t}} \in[0, n]$ tal que $\mathrm{x}(\overline{\mathrm{t}}) \neq 0$.

Logo, $0=\mathrm{p}(\mathrm{V}(\mathrm{x}(\mathrm{t})))<\mathrm{V}(\mathrm{x}(\overline{\mathrm{t}}))$ e temos a propriedade desejada com $T(t)=t-\bar{t}$. 
caso (ii): Se $t=n$ e $x(n)=0$, necessariamente estamos no caso (i-2) devido à propriedade já provada, e segue análogo.

Observemos também o caso em que $x(t)=0$ para todo $t \geq t^{*}$, para algum $t^{*}>0$. Para $t \geq t^{*}, V^{\prime}\left(x_{t}\right)=0$ e devemos provar que valem os itens (i) e (ii) da definiçăo de dicotomia estrita.

Para tanto, seja $M=\sup \left\{T(t): t<t^{*}+1\right\}$.

Para $t \geq t^{*}+1$, tomemos $T(t)=M$. E se $t \geq t^{*}+1+M, \quad t-M \geq t^{*}+1$ com $x_{t-M}=0$; portanto, para estes pontos não é necessário analisar a propriedade de dicotomia estrita.

Concluíndo, para todo $t$ com $V^{\prime}\left(x_{t}\right) \geq 0$, temos $T=M$ onde $M$ é definido como anteriormente.

Assim, os itens (i) e (ii) da Definição de dicotomia estrita (1.14) estão satisfeitos.

Provemos entăo o ítem (iii). Seja $x_{t-T} \rightarrow \bigcap_{s}\left[\Omega_{0}^{\prime}(t, T) \cap \Omega_{o}^{s}(t, T)\right]$ quando $t \rightarrow \infty, s \in(0, T]$.

Logo, $V^{\prime}\left(x_{t}\right) \rightarrow 0$ quando $t \rightarrow \infty$ e $\quad V(x(t))-V(x(t-s)) \rightarrow 0$ quando $t \rightarrow \infty$ para todo $s \in(0, T]$, isto é,

$\lim _{t \rightarrow \infty} V^{\prime}\left(x_{t}\right)=\lim _{t \rightarrow \infty} x(t)[a x(t)+b x([t])]=0 \quad \operatorname{com} x(t) \rightarrow c$ quando $t \rightarrow \infty$,

para alguma constante $\mathbf{c}$.

Então,

$$
\lim _{t \rightarrow \infty} x(t)[\operatorname{ax}(t)+b x([t])]=\lim _{t \rightarrow \infty} c^{2}(a+b)=0 .
$$


Como $(\mathrm{a}+\mathrm{b})<0$, por hipótese, necessariamente $\mathrm{c}=0$ e temos 0 resultado.

Assim, a Definiçăo (1.14) está satisfeita e V é estritamente dicotômica com relação à equaçăo (5.1).

Vamos agora discutir o comportamento da função $\mathrm{V}(\mathrm{x})=\mathrm{x}^{2} / 2$ ao longo da solução da equaçăo discreta associada à (5.1), tomando $\varphi(0)=c_{o}$, utilizando-se da Definição (1.7-A), uma vez que a equação discreta associada é autônoma, como veremos abaixo.

A solução $x(t)$ restrita ao intervalo $[n, n+1), n \in N$, é dada por:

$$
x_{n}(t)=(-b / a) c_{n}+c_{n}(1+b / a) \exp (a(t-n)) \text {, onde } c_{n}=x_{n}\left(n, c_{o}\right) \text {. }
$$

Devido à continuidade de $\mathrm{x}(\mathrm{t})$ nos valores inteiros, devemos ter $c_{n}=x_{n-1}\left(n, c_{o}\right)$, ou seja:

$$
c_{n}=\left[(1+b / a) e^{a}-b / a\right] c_{n-1}=A c_{n-1} .
$$

Se considerarmos a região do $\mathfrak{R}^{2}$ dada por $|A|<1$, que corresponde à $\mathrm{a}\left(1+\mathrm{e}^{\mathrm{a}}\right) /\left(1-\mathrm{e}^{\mathrm{a}}\right)<\mathrm{b}<-\mathrm{a}$ para todo $\mathrm{a} \in \mathfrak{R}, \mathrm{a} \neq 0$, e para $\mathrm{a}=0$ corresponde $\mathrm{a}-2<\mathrm{b}<0$, temos que $\mathrm{V}$ é estritamente dicotômica com relação à equação (5.2) com $\mathrm{k}=2$ conforme Definição (1.7-A).

De fato:

Seja $\Omega$ uma vizinhança arbitrária do zero em $\mathfrak{R}$. Assim:

$$
\Omega_{+}^{1}(2)=\left\{y \in \Omega: \Delta_{1} \mathrm{~V}\left(\mathrm{c}_{2}(\mathrm{y})\right)>0\right\}=\varnothing
$$


pois, para todo $y \in \Omega$ temos: $\quad \Delta_{1} V\left(c_{2}(y)\right)=V\left(c_{2}(y)\right)-V\left(c_{1}(y)\right)=$ $=(1 / 2)\left[c_{2}^{2}(y)-c_{1}^{2}(y)\right]=\left(c_{1}^{2}(y) / 2\right)\left(A^{2}-1\right) \leq 0$, considerando que $|A|<1$.

E também,

$$
\Omega_{\mathrm{o}}^{1}(2)=\left\{\mathrm{y} \in \Omega: \Delta_{1} \mathrm{~V}\left(\mathrm{c}_{2}(\mathrm{y})\right)=0\right\}=\{0\}
$$

pois, $\Delta_{1} V\left(c_{2}(y)\right)=0$ se e somente se, $\mathrm{y}=0$.

Assim, $\left[\bar{\Omega}_{+}^{1}(2) \cup \Omega_{0}^{1}(2)\right]^{*} \subset \Omega_{-}^{2}(2)$.

E mais, $\Omega_{0}^{1}(2) \cap \Omega_{0}^{2}(2)=\{0\}$ pois, se $y \in \Omega_{0}^{1}(2) \cap \Omega_{0}^{2}(2)$, então $\mathrm{V}\left(\mathrm{c}_{2}(\mathrm{y})\right)=\mathrm{V}\left(\mathrm{c}_{1}(\mathrm{y})\right)=\mathrm{V}(\mathrm{y})$.

Assim, $c_{2}^{2}(y)=c_{1}^{2}(y)=y^{2}$ isto é, $\left(A^{2} y\right)^{2}=(A y)^{2}=y^{2}$.

Como $|\mathrm{A}|<1$, necessariamente $\mathrm{y}=0$ e segue o resultado.

Logo, todos os ítens da Definição (1.7-A) estão satisfeitos e portanto, $V$ é estritamente dicotômica com relação à equação (5.2) e pelos Teoremas (2.4) e (3.3), segue que o equilíbrio nulo da equação (5.1) é assintoticamente estável sempre que $\left|(1+b / a) e^{a}-(b / a)\right|<1$.

Observemos que esta região é muito mais abrangente do que a região $\mathrm{a} \leq-\delta<0$ e $|\mathrm{b}|<\mathrm{k} \delta$ para $\delta>0$ e algum $\mathrm{k} \in(0,1)$, considerada inicialmente, principalmente por permitir valores positivos para a constante a.

Portanto, melhores resultados foram possiveis devido ao estudo feito sobre a equação discreta associada (5.2).

Como ilustração, apresentamos o esboço dos gráficos de $x(t)$ e de $\mathrm{V}(\mathrm{x}(\mathrm{t}))$ para $\mathrm{a}=\mathrm{b}=-1$ e $\varphi(0)=\mathrm{c}_{\mathrm{o}}=1 / 2$. 


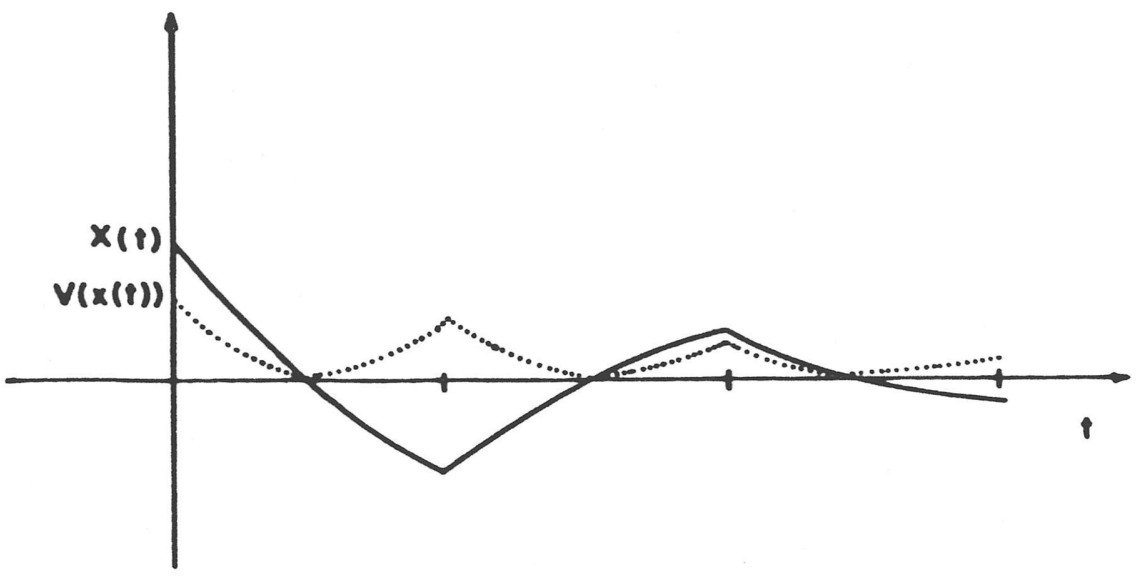

Aplicação 2: Consideremos a equação

$$
x^{\prime}(t)=x([t])-f(t, x(t)) \text { q.t.p., } t \geq t_{0}
$$

onde $f: \mathfrak{R}^{2} \rightarrow \mathfrak{R}$ é contínua, $f(t, 0)=0$ e satisfaz $\times f(t, x) \geq k^{2}$, para algum $\mathrm{k}>1$.

Com estas hipóteses, o equilíbrio nulo de (5.3) é assintoticamente estável.

De fato:

Seja $V(x)=x^{2} / 2$. Assim, $V^{\prime}\left(x_{t}\right)=x(t) x^{\prime}(t)=x(t)[x([t])-f(t, x(t))]$.

Se $t \in Z$ e $x(t) \neq 0$, então $V^{\prime}\left(x_{t}\right)<0$, pois $x(t)=x([t])$ e $V^{\prime}\left(x_{t}\right)=x^{2}(t)-x(t) f(t, x(t)) \leq x^{2}(t)-k x^{2}(t)=(1-k) x^{2}(t)<0 \quad$ considerando que $\mathrm{k}>1$.

Portanto, analisemos os pontos $t \in \Re \backslash Z, t \geq t_{0}$ com $V^{\prime}\left(x_{t}\right) \geq 0$ e $. x(t) \neq 0$. 
Temos que :

$$
V^{\prime}\left(x_{1}\right)=x(t) x([t])-x(t) f(t, x(t)) \leq x(t) x([t])-k x^{2}(t)
$$

Se $V^{\prime}\left(x_{t}\right) \geq 0$, entåo $x(t) x([t])-k x^{2}(t) \geq 0$.

Analisemos os casos $x(t)>0$ e $x(t)<0$.

1) $x(t)>0$

Então, $x([t])-k x(t) \geq 0$, isto é, $x([t]) \geq k x(t)$.

Seja $\mathrm{M}$ uma constante satisfazendo $1<\mathrm{M}<\mathrm{k}$. Assim,

$$
\begin{gathered}
x([t]) \geq \mathrm{kx}(\mathrm{t})>\mathrm{Mx}(\mathrm{t}), \quad \text { ou seja, } \\
\mathrm{x}^{2}([\mathrm{t}]) / 2>\mathrm{M}^{2} \mathrm{x}^{2}(\mathrm{t}) / 2 .
\end{gathered}
$$

Tomando $\mathrm{p}(\mathrm{y})=\mathrm{M}^{2} \mathrm{y}, \mathrm{y}>0$, temos que $\mathrm{p}(\mathrm{V}(\mathrm{x}(\mathrm{t}))<\mathrm{V}(\mathrm{x}([\mathrm{t}]))$.

2) $x(t)<0$

Como $x(t) x([t]))-k x^{2}(t)=-|x(t)|[x([t])+k|x(t)|] \geq 0$, segue que $\mathrm{x}([\mathrm{t}])+\mathrm{k}|\mathrm{x}(\mathrm{t})| \leq 0$, ou seja, $0 \leq \mathrm{k}|\mathrm{x}(\mathrm{t})| \leq-\mathrm{x}([\mathrm{t}])$.

Tomando $\mathrm{M}$ e $\mathrm{p}(\mathrm{t})$ como anteriormente, temos que:

$$
\mathrm{M}|\mathrm{x}(\mathrm{t})|<-\mathrm{x}([\mathrm{t}]) \text {, isto é, } \mathrm{M}^{2} \mathrm{x}^{2}(\mathrm{t}) / 2<\mathrm{x}^{2}([\mathrm{t}]) / 2
$$

Logo, $\mathrm{p}(\mathrm{V}(\mathrm{x}(\mathrm{t})))<\mathrm{V}(\mathrm{x}([\mathrm{t}]))$.

Portanto, sempre que $V^{\prime}\left(x_{t}\right) \geq 0$ e $x(t) \neq 0$ segue que: 
$p(V(x(t)))-V(x([t]))<0$ para $t \in \Re \backslash Z$, e para o caso em que $x(t)=0$, segue análogo à observaçăo feita na Aplicaçăo 1.

Assim, os ítens (i) e (ii) da Definiçăo de dicotomia estrita (1.14) estáo satisfeitos para $\mathrm{T}=\mathrm{M}$ e $\mu\left(\mathrm{t}_{\mathrm{o}}\right)$ arbitrário. Provemos o ítem (iii).

$$
\text { Seja } x_{t-T} \rightarrow \bigcap_{s}\left[\Omega_{0}^{j}(t, T) \cap \Omega_{0}^{s}(t, T)\right] \text { quando } t \rightarrow \infty, s \in(0, T] \text {. }
$$

Entăo, $V^{\prime}\left(x_{t}\left(\cdot, t_{0}, \varphi\right)\right) \rightarrow 0$ e $V(x(t))-V(x(t-s)) \rightarrow 0$ quando $t \rightarrow \infty$, para $s \in(0, T]$.

Assim, $\lim _{t \rightarrow \infty} x(t)=c$ para algum $c$ e $\lim _{t \rightarrow \infty} x(t)[x([t])-f(t, x(t))]=0$.

Como

$$
\begin{gathered}
x(t) x([t])-x(t) f(t, x(t)) \leq x(t) x([t])-k x^{2}(t), \text { então, } \\
0=\lim _{t \rightarrow \infty} x(t)[x([t])-f(t, x(t))] \leq \lim _{t \rightarrow \infty}\left[x(t) x([t])-k x^{2}(t)\right]=c^{2}(1-k) \leq 0,
\end{gathered}
$$

considerando que $(1-\mathrm{k})<0$, e portanto $\mathrm{c}=0$.

Assim, V é estritamente dicotômica com relação à equação (5.3) e pelo Teorema (3.10), tomando $u(x)$ e $v(x)$ como na Aplicação 1, segue 0 resultado.

Aplicação 3: Consideremos a equação:

$$
\begin{aligned}
& x^{\prime}(t)=a^{2}([t])+b x(t) \quad \text { q.t.p., } \quad t \geq 0 \\
& x_{0}=\varphi
\end{aligned}
$$

Se a é uma constante arbitrária năo nula, $b<0$ e $\varphi \in \Omega=\{\psi \in C:\|\psi\|<|\mathrm{b} / \mathrm{a}| \mathrm{k}\}$, para algum $\mathrm{k} \in(0,1)$ e $\mathrm{C}=\mathrm{C}([-1,0), \mathfrak{R})$; temos que o equilíbrio nulo de (5.4) é assintoticamente estável. 
Provemos que $\mathrm{V}(\mathrm{x})=\mathrm{x}^{2} / 2$ é estritamente dicotómica com relaçăo à equaçăo (5.4) em $\Omega$, e através do Teorema (3.10) tomando $u(x)$ e $v(x)$ como nas aplicações anteriores, segue o resultado.

Inicialmente, observermos que para todo $t \in Z_{+} \operatorname{com} x_{1}(\cdot, \varphi) \in \Omega e$ $x(t) \neq 0$, temos que $V^{\prime}\left(x_{1}\right)<0$.

De fato:

Como $x(t)=x([t])$, segue que $V^{\prime}\left(x_{1}\right)=x(t) x^{\prime}(t)=x^{2}(t)[a x(t)+b]$.

Se $x_{t}(\cdot, \varphi) \in \Omega$, então $|x(t)|<|b / a| k$.

Analisemos os casos: $a>0$ e $a<0$.

1) $a>0$

Então, $|x(t)|<-(b / a) k$ e como $k<1$, segue que $x(t)<-b / a \quad e$ portanto, $[\mathrm{ax}(\mathrm{t})+\mathrm{b}]<0$.

2) $a<0$

Então, $|\mathrm{x}(\mathrm{t})|<(\mathrm{b} / \mathrm{a}) \mathrm{k}$, isto é, $(-\mathrm{b} / \mathrm{a}) \mathrm{k}<\mathrm{x}(\mathrm{t})<(\mathrm{b} / \mathrm{a}) \mathrm{k}$.

Como $\mathrm{k}<1$, temos que $-\mathrm{b} / \mathrm{a}<\mathrm{x}(\mathrm{t})$ e portanto, $[\mathrm{ax}(\mathrm{t})+\mathrm{b}]<0$.

Em ambos os casos, $[a x(t)+b]<0$ e segue que $V^{\prime}\left(x_{t}\right)<0$ sempre que $t \in Z_{+} \operatorname{com} x_{t}(\cdot, \varphi) \in \Omega$ e $x(t) \neq 0$.

Sendo assim, basta analisar os pontos $t \in \Re_{+} \backslash Z$ tais que $V^{\prime}\left(x_{t}\right) \geq 0$.

Suponhamos então que $x_{[t]}(\cdot, \varphi) \in \Omega \quad$ com $\quad x(t) \neq 0 \quad$ e $\quad V^{\prime}\left(x_{t}\right) \geq 0$ onde

$$
V^{\prime}\left(x_{t}\right)=x(t)\left[a x^{2}([t])+b x(t)\right]
$$

Analisemos os casos: $\mathrm{x}(\mathrm{t})>0$ e $\mathrm{x}(\mathrm{t})<0$ 
1) $x(t)>0$

Entăo, $a x^{2}([t])+b x(t) \geq 0$.

Se $a>0, x^{2}([t]) \geq(-b / a) x(t) . E$ como $x_{[t]}(\cdot, \varphi) \in \Omega,|x([t])|<(-b / a) k$ e assim,

$$
(-b / a) x(t) \leq x^{2}([t])<(-b / a) k|x([t])|,
$$

e portanto, $x(t) / k<|x([t])|$, ou melhor, $x^{2}(t) / 2 k^{2}<x^{2}([t]) / 2$.

Tomando $\mathrm{p}(\mathrm{y})=\mathrm{y} / \mathrm{k}^{2}, \mathrm{y}>0$, temos que $\mathrm{p}(\mathrm{V}(\mathrm{x}(\mathrm{t})))-\mathrm{V}(\mathrm{x}([\mathrm{t}]))<0$.

Se $a<0, \quad x^{2}([t]) \leq(-b / a) x(t)$. Mas, $(-b / a) x(t) \leq 0$, pois $(-b / a)<0$ e $x(t)>0$.

Logo, $0 \leq \mathrm{x}^{2}([\mathrm{t}]) \leq(-\mathrm{b} / \mathrm{a}) \mathrm{x}(\mathrm{t}) \leq 0$ e portanto $\mathrm{x}(\mathrm{t})=0$; contradição da hipótese tomada. Assim, este caso não ocorre.

2) $x(t)<0$

Então, $a x^{2}([t])+b x(t) \leq 0$.

$\mathrm{Se}$ a $>0$, temos a mesma contradição vista anteriormente.

Se $a<0, x^{2}([t]) \geq(-b / a) x(t)$ e como $x_{[t]}(\cdot, \varphi) \in \Omega,|x([t])|<(b / a) k$, isto é,

$$
(-b / a) x(t) \leq x^{2}([t])<(b / a) k|x([t])| .
$$

Logo, $k|x([t])|>-x(t) \geq 0$, ou seja, $x^{2}([t]) / 2>x^{2}(t) / 2 k^{2}$.

Assim, $\mathrm{p}(\mathrm{V}(\mathrm{x}(\mathrm{t})))-\mathrm{V}(\mathrm{x}([\mathrm{t}]))<0$.

Portanto, em ambos os casos, sempre que $V^{\prime}\left(x_{1}\right) \geq 0$ com $\mathrm{x}_{[t]}(\cdot, \varphi) \in \Omega$ e $\mathrm{x}(\mathrm{t}) \neq 0$, segue que $\mathrm{V}(\mathrm{x}(\mathrm{t}))<\mathrm{p}(\mathrm{V}(\mathrm{x}(\mathrm{t}))<\mathrm{V}(\mathrm{x}([\mathrm{t}]))$.

Assim, para $\varphi \in \Omega$ temos que $\mathrm{x}_{\mathrm{t}}(\cdot, \varphi) \in \Omega$, para todo $\mathrm{t} \geq 0$. 
Concluindo, se $b<0$ e $\varphi \in \Omega^{*}$, sempre que $V^{\prime}\left(x_{1}\right) \geq 0$ com $x(t) \neq 0$ segue que $\mathrm{p}(\mathrm{V}(\mathrm{x}(\mathrm{t})))-\mathrm{V}(\mathrm{x}([\mathrm{t}]))<0$, e o caso $\mathrm{x}(\mathrm{t})=0$ segue análogo à observaçăo feita na Aplicaçăo 1.

Assim, provamos os ítens (i) e (ii) da Definiçăo (1.14) de dicotomia estrita com $\mu$ arbitrário e $\mathrm{T}=\mathrm{M}$.

Provemos agora o ítem (iii). Seja $x_{t-T} \rightarrow \bigcap_{s}\left[\Omega_{0}^{\prime}(t, T) \cap \Omega_{o}^{s}(t, T)\right]$ quando $t \rightarrow \infty, s \in(0, T]$. Então, $V^{\prime}\left(x_{t}(\cdot, \varphi)\right) \rightarrow 0$ e $x(t) \rightarrow c$ quando $t \rightarrow \infty$, para algum c, isto é,

$$
\lim _{t \rightarrow \infty} x(t)\left[a x^{2}([t])+b x(t)\right]=0
$$

Segue que $c^{2}(a c+b)=0$, ou seja, $c=0$ ou $c=-b / a$.

Afirmamos que $c \neq-b / a$, pois para $\varphi \in \Omega$ temos que $x_{t}(\cdot, \varphi) \in \Omega$ para todo $t \geq 0$, isto é, $|x(t, \varphi)|<|b / a| k<|b / a|$.

Assim, $x(t)$ deve convergir, necessariamente para algum valor estritamente menor que $|\mathrm{b} / \mathrm{a}|$ (ou estritamente maior que - $|\mathrm{b} / \mathrm{a}|$ ).

Portanto, c $=0$ e provamos o ítem (iii) da Definiçăo (1.14), concluíndo que $\mathrm{V}$ é estritamente dicotômica com relação à equaçăo (5.4) em $\Omega$.

Aplicaçăo 4: Seja o sistema:

$$
\begin{array}{lll}
x^{\prime}(t)=y(t) x([t]) & \text { q.t.p., } & t \geq 0 \\
y^{\prime}(t)=-x(t) x([t]) & \text { q.t.p., } & t \geq 0
\end{array}
$$


Entáo, o equilíbrio nulo de (5.4-A) é estável.

Provemos que $\mathrm{V}(\mathrm{x}, \mathrm{y})=\left(\mathrm{x}^{2}+\mathrm{y}^{2}\right) / 2$ é dicotómica com relaçăo ao sistema (5.4-A).

Sejam $z_{t}=\left(x_{t}, y_{t}\right)$ e $\Omega$, vizinhança arbitrária da origem em $\mathrm{C}=\mathrm{C}\left([-1,0], \mathfrak{R}^{2}\right)$. Entåo, para todo $\mathrm{t} \geq 0$,

$$
V^{\prime}\left(z_{t}\right)=x(t) x^{\prime}(t)+y(t) y^{\prime}(t)=x(t) y(t) x([t])-x(t) y(t) x([t])=0
$$

Assim, $\Omega_{+}^{\prime}(t, T)=\varnothing$ e $\Omega_{0}^{\prime}(t, T)=\Omega$ para todo $t \geq 0$ com $T>0$ arbitrário. Temos, para $T=1$ que $\Omega_{0}^{1}(t, 1)=\Omega$ para todo $t \geq 1$, pois se $V^{\prime}\left(z_{t}\right)=0$ para todo $t \geq 0$, então $V(z(t))=c$, para algum $c \geq 0$. Assim,

$$
\Omega_{\mathrm{o}}^{1}(\mathrm{t}, 1)=\{\psi \in \Omega: \mathrm{V}(\mathrm{z}(\mathrm{t}, \mathrm{t}-1, \psi))-\mathrm{V}(\mathrm{z}(\mathrm{t}-1, \mathrm{t}-1, \psi))=0\}=\Omega,
$$

para todo $t \geq 1$. Portanto, $\left[\bar{\Omega}_{+}^{\prime}(t, 1) \cup \Omega_{\mathrm{o}}^{\prime}(\mathrm{t}, 1)\right]=\Omega_{\mathrm{o}}^{1}(\mathrm{t}, 1) \subset \bigcup_{s} \Omega_{\mathrm{o}}^{\mathrm{s}}(\mathrm{t}, 1)$, $s \in(0,1]$ e está satisfeita a Definiçăo de dicotomia (1.13) para $\mu=\mathrm{T}=1$, e pelo Teorema (3.10), tomando $u(x)=x^{2} / 4$ e $v(x)=x^{2}$, segue o resultado. Neste caso, năo temos estabilidade assintótica.

Aplicação 5: Consideremos o sistema:

$$
\begin{array}{llll}
x^{\prime}(t)=a x(t)+b x([t]) & \text { q.t.p., } t \geq 0 & x_{0}=\varphi \\
y^{\prime}(t)=c y(t)+d y([t]) & \text { q.t.p., } t \geq 0 & y_{0}=\psi
\end{array}
$$

com as seguintes hipóteses: 


$$
\begin{aligned}
& (a+1 / 2 k)<0 \text { e } \quad(c+1 / 2 k)<0 \\
& \left(2 a+1 / k+b^{2}\right)<0 \text { e }\left(2 c+1 / k+d^{2}\right)<0 \text {, }
\end{aligned}
$$

para algum $k \in(0,1)$.

Nestas condições, o equilíbrio nulo de (5.5) é assintoticamente estável.

Provemos que $\mathrm{V}(\mathrm{x}, \mathrm{y})=\left(\mathrm{x}^{2}+\mathrm{y}^{2}\right) / 2$ é estritamente dicotômica com relaçăo ao sistema (5.5), averigüando a validade da seguinte desigualdade, para todo $\mathrm{t} \in \mathfrak{R}_{+} \backslash \mathrm{Z} \operatorname{com} \mathrm{z}(\mathrm{t}) \neq 0$ :

$$
-\left[\mathrm{V}^{\prime}\left(\mathrm{z}_{\mathrm{t}}\right)+\mathrm{V}(\mathrm{z}(\mathrm{t})) / \mathrm{k}-\mathrm{V}(\mathrm{z}([\mathrm{t}]))\right]>0,
$$

onde $z(t)=(x(t), y(t))$ e $z_{t}=\left(x_{t}, y_{t}\right)$.

Assim, sempre que $V^{\prime}\left(z_{t}\right) \geq 0$, necessariamente teremos que $\mathrm{p}(\mathrm{V}(\mathrm{z}(\mathrm{t})))-\mathrm{V}(\mathrm{z}([\mathrm{t}]))<0$, para todo $\mathrm{t} \in \mathfrak{R}_{+} \backslash \mathrm{Z}$ com $\mathrm{z}(\mathrm{t}) \neq 0$, onde definimos $\mathrm{p}(\mathrm{y})=\mathrm{y} / \mathrm{k}, \mathrm{y}>0$.

E para os valores $t \in Z_{+}$com $z(t) \neq 0$, provaremos que $V^{\prime}\left(z_{t}\right)<0$, e assim, usando a observaçăo feita na Aplicaçăo 1 para o caso $z(t)=0$, teremos provado os ítens (i) e (ii) da Definiçăo de dicotomia estrita (1.14) com $\mu$ arbitrário e $\mathrm{T}=\mathrm{M}$.

1) Provemos que, para $t \in Z_{+} \operatorname{com} z(t) \neq 0, V^{\prime}\left(z_{t}\right)<0$.

Inicialmente, com as hipóteses dadas, notemos que $(a+b)<0$ e $(c+d)<0$, pois: $b^{2}<-2 a-1 / k<-2 a-1<a^{2}$, e portanto, $|b|<|a|=-a$, considerando que a é negativo.

Analogamente, tem-se que $(c+d)<0$.

Seja entăo $t \in Z_{+} \operatorname{com} z(t) \neq 0$. 
Segue que $z(t)=z([t])$ e $V^{\prime}\left(z_{t}\right)=x^{2}(t)(a+b)+y^{2}(t)(c+d)<0$, pois $z(t) \neq 0,(a+b)<0,(c+d)<0$ e segue o resultado.

2) Provemos a validade de (5.6) para $t \in \mathfrak{R}_{+} \backslash Z \operatorname{com} z(t) \neq 0$.

$$
\begin{aligned}
& -V^{\prime}\left(z_{t}\right)-V(z(t)) / k+V(z([t]))=-x(t)[a x(t)+b x([t])]- \\
& -y(t)[c y(t)+d y([t])]-\left[x^{2}(t)+y^{2}(t)\right] / 2 k+\left[x^{2}([t])+y^{2}([t])\right] / 2= \\
& =-(a+1 / 2 k) x^{2}(t)-b x(t) x([t])-(c+1 / 2 k) y^{2}(t)- \\
& \quad-d y(t) y([t])+x^{2}([t]) / 2+y^{2}([t]) / 2 .
\end{aligned}
$$

Esta igualdade pode ser vista como uma forma quadrática $\mathrm{w}^{\mathrm{T}} \mathrm{X} \mathrm{w}$, onde $w(t)=\left[\begin{array}{llll}x(t) & y(t) & x([t]) & y([t])\end{array}\right], e$

$$
\mathrm{X}=\left[\begin{array}{cccc}
-(\mathrm{a}+\mathrm{d} / 2 \mathrm{k}) & 0 & -\mathrm{b} / 2 & 0 \\
0 & -(\mathrm{c}+1 / 2 \mathrm{k}) & 0 & -\mathrm{d} / 2 \\
-\mathrm{b} / 2 & 0 & 1 / 2 & 0 \\
0 & -\mathrm{d} / 2 & 0 & 1 / 2
\end{array}\right]
$$

Nosso objetivo é provar que esta forma quadrática é positiva definida. Para tal, analisemos os menores principais de X, que são dados por:

$$
\begin{gathered}
-(a+1 / 2 k),(a+1 / 2 k)(c+1 / 2 k),(c+1 / 2 k)\left[(a+1 / 2 k) / 2+b^{2} / 4\right] \\
\text { e }\left[(c+1 / 2 k) / 2+d^{2} / 4\right]\left[(a+1 / 2 k) / 2+b^{2} / 4\right] .
\end{gathered}
$$


Como, por hipótese,os fatores $(a+1 / 2 k),(c+1 / 2 k),\left(b^{2}+2 a+1 / k\right)$ e $\left(d^{2}+2 c+1 / k\right)$ sấo negativos, segue que todos os menores principais de $\mathrm{X}$ săo positivos e portanto, a forma quadrática é positiva definida.

Provemos agora o ítem (iii) da Definiçăo (1.14).

Seja $z_{t-T} \rightarrow \bigcap_{s}\left[\Omega_{0}^{\prime}(t, T) \cap \Omega_{0}^{s}(t, T)\right]$ quando $t \rightarrow \infty, s \in(0, T]$.

Entăo, $V^{\prime}\left(z_{t}\right) \rightarrow 0$ e $V(z(t))-V(z(t-s)) \rightarrow 0$ quando $t \rightarrow \infty$ para $s \in(0, T]$. Provemos que $z(t) \rightarrow 0$ quando $t \rightarrow \infty$. Suponhamos que $z(t) \rightarrow c$ quando $\mathrm{t} \rightarrow \infty$, onde $\mathrm{c}=\left(\mathrm{c}_{1}, \mathrm{c}_{2}\right)$.

Temos que $\lim _{\mathrm{t} \rightarrow \infty}\{\mathrm{x}(\mathrm{t})[\mathrm{ax}(\mathrm{t})+\mathrm{bx}([\mathrm{t}])]+\mathrm{y}(\mathrm{t})[\mathrm{cy}(\mathrm{t})+\mathrm{dy}([\mathrm{t}])]\}=0$, com $\lim _{t \rightarrow \infty} z(t)=\lim _{t \rightarrow \infty}(x(t), y(t))=\left(c_{1}, c_{2}\right)$.

Segue que $(a+b) c_{1}^{2}+(c+d) c_{2}^{2}=0$.

Como, por hipótese, $(a+b)<0$ e $(c+d)<0$, então $c_{1}=c_{2}=0$ e temos provado o ítem (iii) da Definição (1.14), concluíndo que $\mathrm{V}$ é estritamente dicotômica com relação ao sistema (5.5).

Aplicação 6: Consideremos o sistema:

$x^{\prime}(t)=a x(t)+b x(t) y^{2}(t)+f(x(t), x([t]))$ q.t.p., $t \geq 0$

$y^{\prime}(t)=c y(t)+d x^{2}(t) y(t)+g(y(t), y([t]))$ q.t.p., $\quad t \geq 0$

satisfazendo as seguintes condições:

(i) $f(x, y)$ e $g(x, y)$ săo contínuas em $\mathfrak{R}^{2}$ com $f(0,0)=g(0,0)=0$,

(ii) a,b,c e d săo constantes com a,c $<-M$, para algum $M>1$ e $(b+d)<0$, 
(iii) $x f(x, y) \leq y^{2} \quad$ e $\quad x g(x, y) \leq y^{2}$, em alguma vizinhança da origem em $\mathfrak{R}^{2}$.

Com estas hipóteses, o equilíbrio nulo de (5.7) é assintoticamente estável.

Provemos que $\mathrm{V}(\mathrm{x}, \mathrm{y})=\left(\mathrm{x}^{2}+\mathrm{y}^{2}\right) / 2$ é estritamente dicotômica com relação ao sistema (5.7).

Primeiramente observemos que, para $t \in Z_{+}$com $z(t) \neq 0$ temos $V^{\prime}\left(z_{t}\right)<0$, onde $z(t)=(x(t), y(t))$.

De fato: Neste caso $z(t)=z([t]) e$

$$
\begin{aligned}
V^{\prime}\left(z_{t}\right)= & a x^{2}(t)+(b+d) x^{2}(t) y^{2}(t)+x(t) f(x(t), x(t))+ \\
& +c y^{2}(t)+y(t) g(y(t), y(t))<(-M+1)\left[x^{2}(t)+y^{2}(t)\right],
\end{aligned}
$$

utilizando-se das hipóteses.

Logo, $V^{\prime}\left(z_{t}\right)<(1-M)\left[x^{2}(t)+y^{2}(t)\right]<0$, considerando que $M>1$ e $z(t) \neq 0$.Sendo assim, basta analisar os valores $t \in \mathfrak{R}_{+} \backslash Z$ com $z(t) \neq 0$, para os quais $V^{\prime}\left(z_{t}\right) \geq 0$. Temos que:

$$
\begin{aligned}
V^{\prime}\left(z_{t}\right)= & a x^{2}(t)+(b+d) x^{2}(t) y^{2}(t)+c y^{2}(t)++x(t) f(x(t), x([t]))+ \\
& +y(t) g(y(t), y([t]))<-M\left[x^{2}(t)+y^{2}(t)\right]+x^{2}([t])+y^{2}([t]),
\end{aligned}
$$

utilizando-se das hipóteses. Logo, se $\mathrm{V}^{\prime}\left(\mathrm{z}_{\mathrm{t}}\right) \geq 0$, segue que:

$$
\left[x^{2}([t])+y^{2}([t])\right] / 2>\left[x^{2}(t)+y^{2}(t)\right] M / 2 .
$$


Definindo $p(y)=M y, y>0$ com $M>1$, temos que $p(V(z(t))<V(z([t])$.

Portanto, sempre que $\mathrm{V}^{\prime}\left(\mathrm{z}_{\mathrm{t}}\right) \geq 0$ com $\mathrm{z}(\mathrm{t}) \neq 0, \mathrm{p}(\mathrm{V}(\mathrm{z}(\mathrm{t}))-\mathrm{V}(\mathrm{z}([\mathrm{t}]))<0$. Se $z(t)=0$, valem as mesmas observaçőes feitas na Aplicaçăo 1 .

Assim, estăo satisfeitos os ítens (i) e (ii) da Definiçăo (1.14) com constante $\mu$ arbitrária, $\mathrm{T}=\mathrm{M}$.

Provemos agora o ítem (iii) da Definiçăo (1.14).

Seja $z_{t-T} \rightarrow \bigcap_{s}\left[\Omega_{0}^{\prime}(t, T) \cap \Omega_{0}^{s}(t, T)\right]$ quando $t \rightarrow \infty, s \in(0, T]$.

Então, $\mathrm{V}^{\prime}\left(\mathrm{z}_{\mathrm{t}}\right) \rightarrow 0$ e $\mathrm{V}(\mathrm{z}(\mathrm{t}))-\mathrm{V}(\mathrm{z}(\mathrm{t}-\mathrm{s})) \rightarrow 0$ quando $\mathrm{t} \rightarrow \infty$, para $s \in(0, T]$. Provemos que $z(t) \rightarrow 0$ quando $t \rightarrow \infty$.

Suponhamos que $z(t) \rightarrow c$ quando $t \rightarrow \infty$, onde $c=\left(c_{1}, c_{2}\right)$. Então, $V^{\prime}\left(z_{t}\right)<-M\left[x^{2}(t)+y^{2}(t)\right]+\left[x^{2}([t])+y^{2}([t])\right]$ com $\lim _{t \rightarrow \infty} V^{\prime}\left(z_{t}\right)=0$. Segue que:

$$
0 \leq \lim _{\mathrm{t} \rightarrow \infty}\left[-\mathrm{M}\left(\mathrm{x}^{2}(\mathrm{t})+\mathrm{y}^{2}(\mathrm{t})\right)+\left(\mathrm{x}^{2}([\mathrm{t}])+\mathrm{y}^{2}([\mathrm{t}])\right)\right]=(1-\mathrm{M})\left(\mathrm{c}_{1}^{2}+\mathrm{c}_{2}^{2}\right) \leq 0,
$$

considerando que $(1-\mathrm{M})<0$.

Portanto, $c_{1}=c_{2}=0$ e temos provado o ítem (iii), concluíndo que $\mathrm{V}$ é estritamente dicotômica com relação ao sistema (5.7).

A seguir, apresentamos exemplos de funçőes que satisfazem a propriedade dada no ítem (iii) da Aplicaçăo 6, em $\Omega=\left\{(x, y) \in \mathfrak{R}^{2}:|x|,|y| \leq 1\right\}$.

Genericamente, $f$ é dada por $f(x, y)=\sum_{i=1}^{m} \lambda_{i} g_{i}(x) y^{n_{i}}$, com: 
$\sum_{i=1}^{m} \lambda_{i}=1$

$g_{1}: \mathfrak{R} \rightarrow \mathfrak{R}$, funçס̋es contínuas satisfazendo $\mathrm{xg}_{1}(\mathrm{x}) \leq 1$, para $|\mathrm{x}| \leq 1$, $\mathrm{i}=1, \cdots, \mathrm{m} \quad \mathrm{e}$

$n_{i} \in N, n_{i} \geq 2, i=1, \cdots, m$.

De fato: Para $(x, y) \in \Omega, x_{i}(x) y^{n_{i}} \leq y^{2}$, para todo $i=1, \cdots, m$. Então,

$$
\lambda_{i} x_{i}(x) y^{n_{i}} \leq \lambda_{i} y^{2} \quad \text { e } \quad x \sum_{i=1}^{m} \lambda_{i} g_{i}(x) y^{n_{i}} \leq y^{2} \sum_{i=1}^{m} \lambda_{i}=y^{2} .
$$

Portanto, $x f(x, y) \leq y^{2}, \forall(x, y) \in \Omega$, e está satisfeita a propriedade do ítem (iii) da Aplicação 6.

Como particulares exemplos, temos:

$$
\begin{aligned}
& f_{1}(x, y)=x^{m} y^{n}, \quad m, n \in N, n \geq 2, \\
& f_{2}(x, y)=y^{2} \operatorname{sen} x / x \quad \text { para }(x, y) \neq(0,0) \text { e } f_{2}(0,0)=0, \\
& f_{3}(x, y)=y^{2}(\cos x-1) / 2 x \text { para }(x, y) \neq(0,0) \text { e } f_{3}(0,0)=0 \text { e } \\
& f_{4}(x, y)=y^{2} x \operatorname{sen}(1 / x) \quad \text { para }(x, y) \neq(0,0) \text { e } f_{4}(0,0)=0 .
\end{aligned}
$$

\section{Aplicação 7:}

Seja a funçăo $\mathrm{f}:[0,2 \pi) \times \Re_{+} \rightarrow \Re$ onde $\mathrm{f}(\theta, \mathrm{r})=\mathrm{r} \exp (-\mathrm{r} \cos \theta)$.

Dada uma seqüência $\left(\theta_{n}\right)_{n \in \mathbb{N}}$, denotemos $r_{n}=r\left(\theta_{n}\right)$ e consideremos a equação discreta $r_{n+1}=f\left(\theta_{n}, r_{n}\right), n \in N$.

Em particular, para $\theta_{n+1}=\theta_{n}+\pi / 2, n \in N$, a equação discreta correspondente tem a seguinte forma: 


$$
r_{n+1}=r_{n} \exp \left(-r_{n} \cos \theta_{n}\right), n \in N,
$$

com valor inicial $r_{0}=r$, e aqui, $\theta$ desempenha o papel da variável "tempo", medido segundo a lei acima.

Como o zero é ponto de equilibrio de (5.8), estudemos a sua estabilidade.

Provemos que $V(r)=r^{2}$ é estritamente dicotômica com relaçăo à equação (5.8) em $\Omega=\left\{r \in R_{+}: r^{r}<1\right\}$ e escolhendo $u(r)=r^{2} / 2$ e $v(r)=r^{2}$, pelo Teorema (3.3) temos que o equilíbrio nulo de (5.8) é assintoticamente estável.

Suponhamos que $\Delta_{1} V\left(r_{4}(r)\right) \geq 0$ para $r \in \Omega^{*}$.

Então, $\Delta_{1} V\left(r_{4}(r)\right)=V\left(r_{4}(r)\right)-V\left(r_{3}(r)\right) \geq 0$, ou seja, $r_{3} \leq r_{4}$.

Dai, $r_{3} \leq r_{3} \exp \left(-r_{3} \cos \theta_{3}\right)$ e assim, $\cos \theta_{3} \leq 0$.

Temos dois casos a analisar: $\theta_{3} \in[\pi / 2, \pi]$ e $\theta_{3} \in[\pi, 3 \pi / 2]$.

1) $\theta_{3} \in[\pi / 2, \pi]$. Provemos que $\Delta_{3} V\left(r_{4}(r)\right)<0$.

De fato:

$$
\begin{aligned}
r_{2} & =r_{1} \exp \left(-r_{1} \cos \theta_{1}\right), \\
r_{3} & =r_{2} \exp \left(-r_{2} \cos \theta_{2}\right)=r_{1} \exp \left(-r_{1} \cos \theta_{1}\right) \exp \left[-r_{1} \exp \left(-r_{1} \cos \theta_{1}\right) \cos \theta_{2}\right]= \\
& =r_{1} \exp \left(-A r_{1}\right), \text { onde } A=\cos \theta_{1}+\exp \left(-r_{1} \cos \theta_{1}\right) \cos \theta_{2}, \\
r_{4} & =r_{3} \exp \left(-r_{3} \cos \theta_{3}\right)=r_{1} \exp \left(-A r_{1}\right) \exp \left[-r_{1} \exp \left(-A r_{1}\right) \cos \theta_{3}\right]= \\
& =r_{1} \exp \left(-B r_{1}\right), \text { onde } B=A+\exp \left(-A r_{1}\right) \cos \theta_{3} .
\end{aligned}
$$

Observemos que, se $B>0$, segue que $r_{4}<r_{1}$, e portanto, $\Delta_{3} \mathrm{~V}\left(\mathrm{r}_{4}(\mathrm{r})\right)=\mathrm{V}\left(\mathrm{r}_{4}\right)-\mathrm{V}\left(\mathrm{r}_{1}\right)=\mathrm{r}_{4}^{2}-\mathrm{r}_{1}^{2}<0$, que é o resultado desejado.

Provemos entăo que $B>0$, sempre que $r \in \Omega^{\circ}$. 
Inicialmente mostremos que, para $r \in \Omega^{\circ}, A \geq 1$ e $A-1<B \leq A$ e assim, $0 \leq A-1<B \leq A$ e segue que $B>0$.

a) $\mathrm{A} \geq 1$

De fato:

Como $\theta_{2}=\theta_{1}+\pi / 2$, então $A=\cos \theta_{1}-\operatorname{sen} \theta_{1} \exp \left(-r_{1} \cos \theta_{1}\right)$.

Se $r^{r}<1$, entăo $r_{1}<1$, pois $r_{1}=r \exp (-r \cos \theta)$ onde $\theta \in[\pi, 3 \pi / 2]$, e assim, $\mathrm{r}_{1} \leq \mathrm{re}^{\mathrm{r}}$.

E considerando que $\cos \theta_{1} \in[0,1]$ e $\operatorname{sen} \theta_{1} \in[-1,0]$, temos:

$\cos \theta_{1}-\operatorname{sen} \theta_{1} \geq \cos \theta_{1}-\operatorname{sen} \theta_{1} \exp \left(-r_{1} \cos \theta_{1}\right) \geq \cos \theta_{1}-\operatorname{sen} \theta_{1} \exp \left(-\cos \theta_{1}\right)$, ou seja, $A \geq \cos \theta_{1}-\operatorname{sen} \theta_{1} \exp \left(-\cos \theta_{1}\right)$.

Provemos que $f\left(\theta_{1}\right)=\cos \theta_{1}-\operatorname{sen} \theta_{1} \exp \left(-\cos \theta_{1}\right)-1 \geq 0$, e assim segue que $A-1 \geq 0$.

Se $\cos \theta_{1}=x, \operatorname{sen} \theta_{1}=-\sqrt{1-x^{2}}$ para $\theta_{1} \in[3 \pi / 2,2 \pi]$.

Então, $f(x)=x+\sqrt{1-x^{2}} \exp (-x)-1$.

Afirmamos que $f(x) \geq 0$ para $x \in[0,1]$, pois a desigualdade equivalente dada por $\mathrm{xe}^{\mathrm{x}} \geq \mathrm{e}^{\mathrm{x}}-\sqrt{1-\mathrm{x}^{2}}$ é verdadeira no intervalo considerado, bastando para isso comparar os gráficos das funções.

b) A $-1<$ B $\leq$ A

Considerando que $r_{1} \in(0,1), \quad A \geq 1, \quad \cos \theta_{3} \in[-1,0]$ e que $\mathrm{B}=\mathrm{A}+\exp \left(-\mathrm{Ar}_{1}\right) \cos \theta_{3}$, temos que $\mathrm{A}-\exp \left(-\mathrm{Ar}_{1}\right) \leq \mathrm{B} \leq \mathrm{A}$.

E como $A-1<A-\exp \left(-A r_{1}\right)<A-\exp (-A)$, segue que:

$$
\mathrm{A}-1<\mathrm{A}-\exp \left(-\mathrm{Ar}_{1}\right) \leq \mathrm{B} \leq \mathrm{A} \text {, ou seja, } \mathrm{A}-1<\mathrm{B} \leq \mathrm{A} \text {. }
$$

Portanto, a partir de (a) e (b), temos que B $>0$ para $r \in \Omega^{*}$, e assim, $\Delta_{3} \mathrm{~V}\left(\mathrm{r}_{4}(\mathrm{r})\right)<0$. 
2) $\theta_{3} \in[\pi, 3 \pi / 2]$. Provemos que $\Delta_{4} V\left(r_{4}(r)\right)<0$.

De fato:

$$
\begin{aligned}
& r_{1}=r \exp (-r \cos \theta), \\
& r_{2}=r_{1} \exp \left(-r_{1} \cos \theta_{1}\right)=r \exp (-A r), \text { onde } A=\cos \theta+\cos \theta_{1} \exp (-r \cos \theta), \\
& r_{3}=r_{2} \exp \left(-r_{2} \cos \theta_{2}\right)=r \exp (-B r), \text { onde } B=A+\cos \theta_{2} \exp (-A r) e \\
& r_{4}=r_{3} \exp \left(-r_{3} \cos \theta_{3}\right)=r \exp (-C r), \text { onde } C=B+\cos \theta_{3} \exp (-B r) .
\end{aligned}
$$

Observemos que se $C>0$, segue que $r_{4}<r$, e portanto, $\Delta_{4} V\left(r_{4}(r)\right)=V\left(r_{4}\right)-V(r)=r_{4}^{2}-r^{2}<0$ para $r \in \Omega^{*}$, que é $\circ$ resultado desejado.

Para análise do gráfico da função $C=C\left(r, \theta_{2}\right)$ em $(0,1) \times[\pi / 2, \pi]$, recorremos ao programa computacional "Mathematica" pois, algebricamente, a análise do sinal da referida função requer muito cálculo. Segue abaixo o gráfico da função $C$.
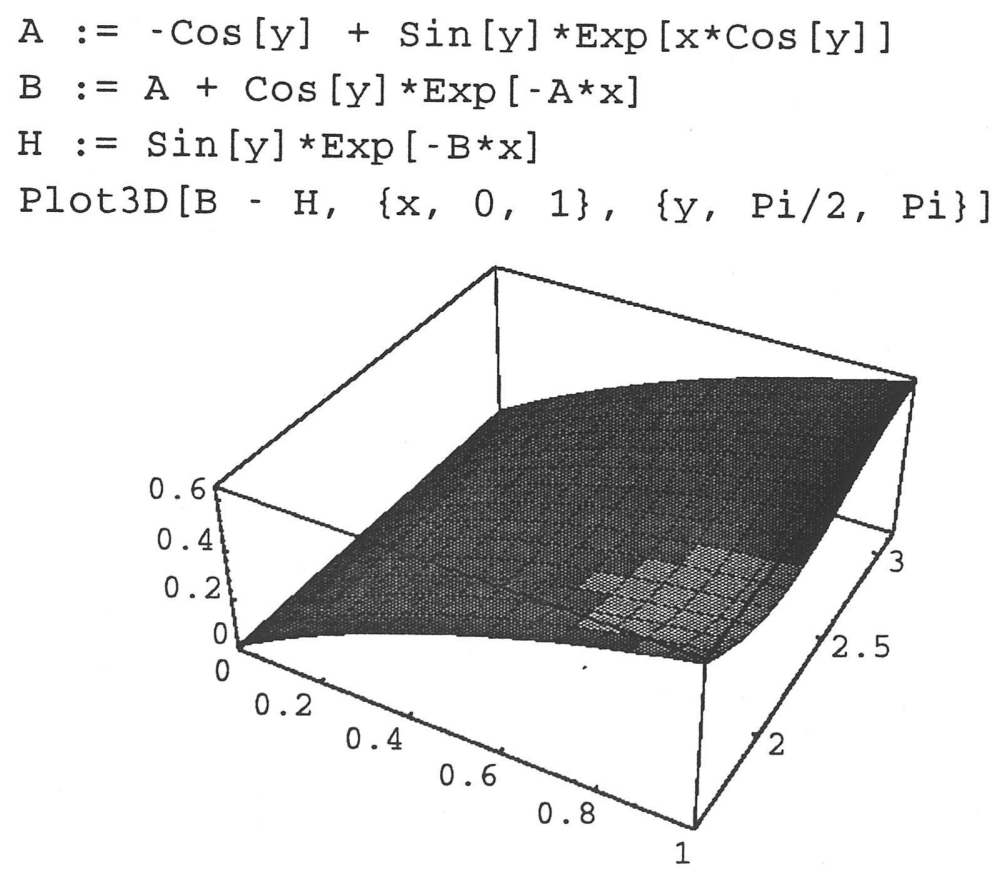

- SurfaceGraphics - 
Observemos que $C\left(r, \theta_{2}\right)=0$ somente se $r=0$ e é positiva para os demais valores do seu domínio.

Portanto, se $C>0$ para $r \in \Omega^{*}$, segue que $\Delta_{4} V\left(r_{4}(r)\right)<0$.

Assim, dada a vizinhança $\Omega$ da origem em $R$, existe $k=4$ tal que: sempre que $\Delta_{1} V\left(r_{4}(r)\right) \geq 0$ para $r \in \Omega^{*}$, segue que $\Delta_{j} V\left(r_{4}(r)\right)<0$, para $j=3$ ou $j=4$, ou seja:

$$
\left[\bar{\Omega}_{+}^{1}(4) \cup \Omega_{\mathrm{o}}^{1}(4)\right]^{\bullet} \subset \bigcup_{j=2}^{4} \Omega_{-}^{j}(4) .
$$

Logo, o ítem (i) da Definição de dicotomia estrita (1.7-A) está satisfeito; provemos o ítem (ii), isto é, $\Omega_{0}^{1}(4) \cap \Omega_{0}^{4}(4)=\{0\}$.

De fato:

Se $r \in \Omega_{0}^{1}(4) \cap \Omega_{0}^{4}(4)$, então $V\left(r_{4}(r)\right)=V\left(r_{3}(r)\right)=V(r)$, ou seja, $r_{4}=r_{3}=r$.

Mas, $r_{4}=r \exp (-\mathrm{Cr})$ e $r=r \exp (-\mathrm{Cr})$ somente se $r=0$, pois $\mathrm{C}$ é sempre positivo para $r \neq 0$. Assim, todos os ítens da Definição (1.7-A) estão satisfeitos e portanto, $\mathrm{V}$ é estritamente dicotômica com relaçăo à (5.8).

\section{Aplicação 8:}

Conforme as considerações feitas na Aplicação 7, seja a seguinte equação discreta:

$$
r_{n+1}=a(n) r_{n} \exp \left[-\cos \theta_{n} /(n+1)\right], n=n_{o}, n_{o}+1, \cdots
$$

$\operatorname{com} r_{n_{o}}=r, \theta_{n+1}=\theta_{n}+\pi / 2, n \geq n_{o}, n_{o} \in N$, 
$a: \Re \rightarrow \Re$, é uma funçăo contínua, $a(t)<k<1$ para todo $t \in \mathfrak{R}$, para alguma constante $k$ com $\lim _{t \rightarrow \infty} a(t)=d \neq 1$.

Com estas hipóteses, o equilibrio nulo de (5.9) é assintoticamente estável.

Provemos que $V(r)=r^{2}$ é estritamente dicotômica com relaçăo à (5.9).

Suponhamos que $V\left(r_{n-1}\right) \leq V\left(r_{n}\right)$, para algum $n>n_{0}$, com $r_{n-4} \neq 0$.

Então $r_{n-1} \leq r_{n}$, isto é, $r_{n-1} \leq r_{n-1} a(n-1) \exp \left[-\cos \theta_{n-1} / n\right]$, e assim segue que $\cos \theta_{n-1} \leq 0$.

Temos dois casos a considerar: $\theta_{n-1} \in[\pi / 2, \pi]$ e $\theta_{n-1} \in[\pi, 3 \pi / 2]$.

1) $\theta_{n-1} \in[\pi / 2, \pi]$

$$
\begin{aligned}
& r_{n-2}= r_{n-3} a(n-3) \exp \left[-\cos \theta_{n-3} /(n-2)\right], \\
& r_{n-1}= r_{n-2} a(n-2) \exp \left[-\cos \theta_{n-2} /(n-1)\right]= \\
&= r_{n-3} a(n-3) a(n-2) \exp \left\{-\cos \theta_{n-3} /(n-2)-\cos \theta_{n-2} /(n-1)\right\}, \\
& r_{n}= r_{n-1} a(n-1) \exp \left[-\cos \theta_{n-1} / n\right]=r_{n-3} a(n-3) a(n-2) a(n-1) \exp (B), \text { onde } \\
& \quad B=-\cos \theta_{n-3} /(n-2)-\cos \theta_{n-2} /(n-1)-\cos \theta_{n-1} / n .
\end{aligned}
$$

Observemos inicialmente que $\mathrm{B}<0$.

Como $\theta_{n-1} \in[\pi / 2, \pi]$, então $\cos \theta_{n-2}=-\operatorname{sen} \theta_{n-3}$ e $\cos \theta_{n-1}=-\cos \theta_{n-3}$.

Assim, $B=-\cos \theta_{n-3} /(n-2)+\operatorname{sen} \theta_{n-3} /(n-1)+\cos \theta_{n-3} / n$, ou seja, $B=\left[-2(n-1) \cos \theta_{n-3}+n(n-2) \operatorname{sen} \theta_{n-3}\right] / n(n-1)(n-2)$.

Chamando $x=\cos \theta_{n-3} \operatorname{com} \theta_{n-3} \in[3 \pi / 2,2 \pi]$, temos que $x \in[0,1]$ e que $\operatorname{sen} \theta_{\mathrm{n} \cdot 3}=-\sqrt{1-\mathrm{x}^{2}}$.

Logo, $\quad B(n, x)=-\left[2(n-1) x+n(n-2) \sqrt{1-x^{2}}\right] / n(n-1)(n-2)$ portanto, $B(n, x)<0$ para $n \geq n_{0}+3$ e $x \in[0,1]$. 
Assim, como $r_{n}=r_{n-3} a(n-3) a(n-2) a(n-1) e^{B}, \operatorname{com} a(n)<k<1$ para todo $n \in N$, e $B<0$, temos que:

$$
r_{n}^{2} / k<\left[r_{n-3}^{2} k^{6} e^{2 B}\right] / k<r_{n-3}^{2} .
$$

Chamando $p(y)=y / k, y>0$, segue que $p\left(V\left(r_{n}\right)\right)<V\left(r_{n-3}\right)$.

2) $\theta_{n-1} \in[\pi, 3 \pi / 2]$

$$
\begin{aligned}
r_{n-3}= & r_{n-4} a(n-4) \exp \left[-\cos \theta_{n-4} /(n-3)\right], \\
r_{n-2}= & r_{n-3} a(n-3) a(n-4) \exp \left[-\cos \theta_{n-4} /(n-3)-\cos \theta_{n-3} /(n-2)\right], \\
r_{n-1}= & r_{n-2} a(n-2) a(n-3) a(n-4) \exp \left[-\cos \theta_{n-4} /(n-3)-\cos \theta_{n-3} /(n-2)-\right. \\
& \left.\quad-\cos \theta_{n-2} /(n-1)\right], \\
r_{n}= & r_{n-4} a(n-1) a(n-2) a(n-3) a(n-4) \exp (A), \text { onde: } \\
\quad A= & -\cos \theta_{n-4} /(n-3)-\cos \theta_{n-3} /(n-2)-\cos \theta_{n-2} /(n-1)-\cos \theta_{n-1} / n .
\end{aligned}
$$

Inicialmente, mostremos que $\mathrm{A}<0$.

Como $\theta_{\mathrm{n}-1} \in[\pi, 3 \pi / 2]$, então $\cos \theta_{\mathrm{n}-1}=-\operatorname{sen} \theta_{\mathrm{n}-2}, \cos \theta_{\mathrm{n}-4}=-\cos \theta_{\mathrm{n}-2} \quad \mathrm{e}$ $\cos \theta_{\mathrm{n}-3}=\operatorname{sen} \theta_{\mathrm{n}-2}$.

Assim, $\mathrm{A}=2 \cos \theta_{\mathrm{n}-2} /(\mathrm{n}-1)(\mathrm{n}-3)-2 \operatorname{sen} \theta_{\mathrm{n}-2} / \mathrm{n}(\mathrm{n}-2), \quad \mathrm{e}$ portanto $A<0$ pois $\cos \theta_{n-2} \in[-1,0]$ e $\operatorname{sen} \theta_{n-2} \in[0,1] \operatorname{com} n \geq n_{o}+4$.

Como $r_{n}=r_{n-4} a(n-1) a(n-2) a(n-3) a(n-4) e^{A}$, temos que:

$$
\mathrm{r}_{\mathrm{n}}^{2} / \mathrm{k}<\left[\mathrm{r}_{\mathrm{n}-4}^{2} \mathrm{k}^{8} \mathrm{e}^{2 \mathrm{~A}}\right] / \mathrm{k}<\mathrm{r}_{\mathrm{n}-4}^{2} \text {. Então, } \mathrm{p}\left(\mathrm{V}\left(\mathrm{r}_{\mathrm{n}}\right)\right)<\mathrm{V}\left(\mathrm{r}_{\mathrm{n}-4}\right)
$$

Logo, em ambos os casos, sempre que $V\left(r_{n}\right)-V\left(r_{n-1}\right) \geq 0$ segue que $p\left(V\left(r_{n}\right)\right)-V\left(r_{n-j}\right)<0$ para $n \geq n_{o}+4$ e $r_{n-4} \neq 0$, onde $j=3$ ou $j=4$. 
Assim, os îtens (i) e (ii) da Definiçăo de dicotomia estrita (1.7) estăo satisfeitos com $M=4$ e $N=4$.

Provemos o ítem (iii). Seja $r_{n-k}(r) \rightarrow \bigcap_{j=1}^{k} \Omega_{0}^{j}(n, k)$ quando $n \rightarrow \infty$.

Entăo $V\left(r_{n}\right)-V\left(r_{n-j}\right) \rightarrow 0$ quando $n \rightarrow \infty$ para $j=1, \cdots, k$, em particular, para $j=1, r_{n}^{2}-r_{n-1}^{2} \rightarrow 0$ quando $n \rightarrow \infty$.

Logo,

$$
\lim _{n \rightarrow \infty} r_{n-1}^{2}\left[a^{2}(n-1) \exp \left(-2 \cos \theta_{n-1} / n\right)-1\right]=0 .
$$

Provemos que $r_{n}(r) \rightarrow 0$ quando $n \rightarrow \infty$. Suponhamos que $r_{n}(r) \rightarrow c$ quando $\mathrm{n} \rightarrow \infty$, para alguma constante $\mathrm{c} \geq 0$.

$$
\text { Como } \begin{aligned}
& \lim _{n \rightarrow \infty} a(n)=d \quad e \\
& \lim _{n \rightarrow \infty} \exp \left[-\cos \theta_{n-1} / n\right]=\lim _{n \rightarrow \infty} \exp \left\{-\cos \left[\theta_{0}+(n-1) \pi / 2\right] / n\right\}=1,
\end{aligned}
$$

segue que $\lim _{n \rightarrow \infty} r_{n-1}^{2}\left[a^{2}(n-1) \exp \left\{-2 \cos \theta_{n-1} / n\right\}-1\right]=c^{2}\left(d^{2}-1\right)=0$.

Mas como $d \neq 1$, por hipótese, necessariamente $c=0$ e portanto, a Definiçăo (1.7) está satisfeita, isto é, V é estritamente dicotômica com relaçăo à (5.9) com $\mathrm{N}=4$ e para cada $n \geq n_{0}, k(n)=3$ ou 4 .

Escolhemos as funçס̃es u e v como na Aplicação 7 e pelo Teorema (3.3), o equílibrio nulo de (5.9) é assintoticamente estável. 


\section{BIBLIOGRAFIA}

[1] AFTABIZADEH, A.R.; WIENER, J. Oscillatory and periodic solutions for systems of two first order linear differential equations with piecewise constant argument. Applic. Anal., v.26, p.327-333, 1988.

[2] BENÁ, M.A. Estabilidade de equações diferenciais retardadas através do método das funções dicotômicas. São Carlos, ICMSC-USP, 1993 (Tese de Doutorado).

[3] CARVALHO, L.A.V. The stability of discrete equations and ordinary differential equations. Delay Differential Equations and Dynamical Systems. New York, Springer-Verlag, p.88-97, 1990.

[4] CARVALHO, L.A.V.; COOKE, K.L. On dichotomic maps for a class of differential-difference equations. Proc. Royal Soc. Edinburgh, v.117A, p.317-328, 1991.

[5] CARVALHO, L.A.V. ; FERREIRA, R.R. On a new extension of Liapunov's direct method to discrete equations. Quart. Appl. Math., v.46, n.4, p.779-788, 1988.

[6] COOKE, K.L.; WIENER, J. A survey of differential equations with piecewise continuous arguments. Delay Differential Equations and Dynamical Systems. New York, Springer-Verlag, p.1-15, 1990. 
[7] Neutral differential equations with piecewise constant arguments. Boll. Un. Mat. Ital. (7)1-B, p.321-346, 1987.

[8] Oscillations in systems of differential equations with piecewise constant argument. J. Math. Anal. Appl., v.137, p.221-239, 1989.

[9] Retarded differential equations with piecewise constant delays. J. Math. Anal. Appl., v.99, p.265-297, 1984.

[10] Stability regions for linear equations with piecewise continuous delay. Comp. \& Math. with App/s., v.12A, n.6, p.695-701, 1986.

[11] DRIVER, R.D. Ordinary and delay differential equations. New York, Springer-Verlag, 1977 (Appl. Math. Sciences, 20).

[12] HALE, J.K. Ordinary differential equations. New York, Robert E. Krieger Publis. Company, 1980.

[13] Theory of functional differential equations. New York, Springer-Verlag, 1977.

[14] HURT, J. Some stability theorems for ordinary difference equations. SIAM J. Numerical Anal. 4, p.582-596, 1967. 
[15] JAYASREE, K.N.; DEO, S.G. On piecewise constant delay differential equations. J. Math. Anal. and Appl., v.169, p.55-69, 1992.

[16] LA SALLE, J.P. Stability theory for difference equations. MAA Stu. in Math. v.14, p.1-31, 1977.

[17] MC SHANE, E.J. Integration. Princeton, Princeton University Press, 1947.

[18] MYSHKIS, A.D. On certain problems in the theory of differential equations with deviating argument. Uspekhi Mat. Nauk., 32, p.173202, 1977.

[19] ROYDEN, H.L. Real Analysis, London, The Macmillan Company.

[20] SEIFERT, G. Certain systems with piecewise constant feedback controls with a time delay. Diff. and Integ. Eq., v.6, n.4, p.937-947, 1993. 


\section{APENDICE}

Resultado: Se $f: \mathfrak{R} \times \mathfrak{R}^{2 \mathrm{n}} \rightarrow \mathfrak{R}^{\mathrm{n}}$ é contínua, a funçăo $\mathrm{g}: \mathfrak{R} \times \mathrm{C} \rightarrow \mathfrak{R}^{\mathrm{n}}$ dada por:

$$
g(t, \varphi)=f(t, \varphi(0), \varphi([t]-t)), \quad t \geq t_{0}
$$

satisfaz condição de Carathéodory sobre $\Re$ x C.

Demonstração: Usaremos em $\mathfrak{R} \times \mathrm{C}$ a norma da soma, em $\mathrm{C}=\mathrm{C}\left([-1,0], \mathfrak{R}^{\mathrm{n}}\right)$ a norma do supremo, e em $\mathfrak{R}^{2 \mathrm{n}}$ a norma do máximo.

1) Provemos que $\mathrm{g}(\mathrm{t}, \varphi)$ é mensurável em $\mathrm{t}$, para cada $\varphi$ fixa.

Definimos as funções:

$$
\begin{aligned}
& \mathrm{k}: \mathfrak{R} \rightarrow[-1,0], \quad \mathrm{k}(\mathrm{t})=[\mathrm{t}]-\mathrm{t} \quad \mathrm{e} \\
& \mathrm{H}: \mathfrak{R} \rightarrow \mathfrak{R} \times \Re^{\mathrm{n}} \quad, \quad \mathrm{H}=(\text { Id }, \varphi \circ \mathrm{k}) .
\end{aligned}
$$

Assim, para cada $\varphi \in \mathrm{C}$ fixa, temos:

$$
g(t, \varphi)=f(t, \varphi(0), \varphi([t]-t))=f_{\varphi_{(0)}}(t, \varphi([t]-t))=\left(f_{\varphi_{(0)}} \circ H\right)(t)
$$

onde $f_{\varphi_{(0)}}$ denota a função $f$ com a segunda variável fixa.

Portanto,

$$
g_{\varphi}(t)=\left(f_{\varphi_{(0)}} \circ H\right)(t), \quad \text { para todo } t \in \mathfrak{R}
$$


Como k é mensurável, f e $\varphi$ săo contínuas, segue que $g_{\Phi}$ é mensurável, para cada $\varphi$ fixa.

2) Provemos que g é contínua em $\varphi$, para cada t fixo.

Como $f$ é contínua, para cada t fixo e $\left(\mathrm{x}_{0}, \mathrm{y}_{0}\right) \in \mathfrak{R}^{\mathrm{n}} \mathrm{x} \mathfrak{R}^{\mathrm{n}}$, dado $\varepsilon>0$, existe $\delta>0$ tal que se $\left|(x, y)-\left(x_{0}, y_{0}\right)\right|<\delta$, para $(x, y) \in \mathfrak{R}^{\mathrm{n}} x \mathfrak{R}^{\mathrm{n}}$, entăo $\left|f(t, x, y)-f\left(t, x_{0}, y_{o}\right)\right|<\varepsilon$.

Seja $\varphi_{1} \in \mathrm{C}$. Tomemos $\varphi \in \mathrm{C}$ tal que $\left\|\varphi-\varphi_{1}\right\|<\delta$.

Logo, para cada t fixo,

$$
\begin{aligned}
& \left\|\varphi(0)-\varphi_{1}(0)\right\|<\delta \quad e \\
& \left\|\varphi([\mathrm{t}]-\mathrm{t})-\varphi_{1}([\mathrm{t}]-\mathrm{t})\right\|<\delta .
\end{aligned}
$$

Então, $\mid\left(\varphi(0), \varphi([t]-t)-\left(\varphi_{1}(0), \varphi_{1}([t]-t)\right) \mid<\delta\right.$, e pela hipótese sobre $f$, segue que

$$
\left|g(t, \varphi)-g\left(t, \varphi_{1}\right)\right|=\left|f(t, \varphi(0), \varphi([t]-t))-f\left(t, \varphi_{1}(0), \varphi_{1}([t]-t)\right)\right|<\varepsilon,
$$

para cada t fixo.

Portanto, para cada t fixo e $\varphi_{1} \in \mathrm{C}$, temos:

Dado $\varepsilon>0, \exists \delta>0 /$ se $\left\|\varphi-\varphi_{1}\right\|<\delta$ para $\varphi \in C$, então $\left|\mathrm{g}(\mathrm{t}, \varphi)-\mathrm{g}\left(\mathrm{t}, \varphi_{1}\right)\right|<\varepsilon$, o que prova o resultado.

3) Para todo $(t, \varphi) \in \mathfrak{R} \times \mathrm{C}$ fixo, provemos que existe uma vizinhança $\mathrm{V}(\mathrm{t}, \varphi)$ e uma função Lebesgue Integrável $\mathrm{m}$, tal que: 


$$
|\mathrm{g}(\mathrm{s}, \psi)| \leq \mathrm{m}(\mathrm{s}) \quad, \quad(\mathrm{s}, \psi) \in \mathrm{V}(\mathrm{t}, \varphi)
$$

De fato:

Sejam $(t, \varphi) \in \mathfrak{R} \times \mathrm{C}$ e $\varepsilon>0$. Tomemos $\delta>0$ dado pela continuidade de $f$ e definimos:

$$
\mathrm{V}_{\delta}(\mathrm{t}, \varphi)=\{(\mathrm{s}, \psi) \in \mathfrak{R} \times \mathrm{C}:|(\mathrm{t}, \varphi)-(\mathrm{s}, \psi)|<\delta\}
$$

Assim, para cada s tal que $(s, \psi) \in \mathrm{V}_{\delta}(\mathrm{t}, \varphi)$, temos que $\|\varphi-\psi\|<\delta$, ou:

$$
\begin{gathered}
|\varphi(0)-\psi(0)|<\delta \quad \text { e } \\
|\varphi([s]-s)-\psi([s]-s)|<\delta,
\end{gathered}
$$

o que é equivalente à $|(s, \psi(0), \psi([s]-s))-(s, \varphi(0), \varphi([s]-s))|<\delta$.

E pela continuidade de $\mathrm{f}$, segue que:

$$
\begin{aligned}
& |f(s, \psi(0), \psi([s]-s))-f(s, \varphi(0), \varphi([s]-s))|<\varepsilon, \text { ou } \\
& \quad|f(s, \psi(0), \psi([s]-s))|<\varepsilon+|f(s, \varphi(0), \varphi([s]-s))| .
\end{aligned}
$$

Logo, para $(t, \varphi) \in \Re \times C$, fixo e $\varepsilon=1$, existe uma vizinhança $V_{\delta}(t, \varphi)$ e uma funçăo m dada por:

$$
m(s)=1+|f(s, \varphi(0), \varphi([s]-s))|
$$


tal que

$$
|g(s, \psi)|=|f(s, \psi(0), \psi([s]-s))|<m(s) \text {, para }(s, \psi) \in V_{\delta}(t, \varphi) \text {. }
$$

Para concluir o resultado desejado, basta provar que a funçăo $\mathrm{m}$ é Lebesgue Integrável.

Notemos que o domínio de $m$ é o intervalo $(t-\delta, t+\delta)$ pois, para cada $\mathrm{s}$ com $(\mathrm{s}, \psi) \in \mathrm{V}_{\delta}(\mathrm{t}, \varphi)$, temos que $|\mathrm{s}-\mathrm{t}|<\delta$.

Consideremos dois casos: $t \notin N$ e $t \in N$.

1) $\mathrm{t} \notin N$

Se $t \in(n, n+1)$, para algum $n \in N$, a função $k(s)=[s]-s$ torna-se contínua para $\mathrm{s} \in(\mathrm{t}-\delta, \mathrm{t}+\delta)$, o que leva à continuidade de $\mathrm{m} e$ conseqüentemente, $\mathrm{m}$ é Lebesgue Integrável, considerando que $\mathrm{m}$ é limitada sobre seu domínio.

2) $t \in N$

Neste caso, $k$ é contínua à esquerda em $(t-\delta, t]$ e contínua em $[t, t+\delta)$.

Seja uma nova função $\ell:(t-\delta, t] \rightarrow \Re$, dada por:

$$
\ell(\mathrm{s})= \begin{cases}\mathrm{k}(\mathrm{s}) & , \mathrm{t}-\delta<\mathrm{s}<\mathrm{t} \\ -1, & \mathrm{~s}=\mathrm{t}\end{cases}
$$

Temos que $\ell=\mathrm{k}$ em quase toda parte de $(\mathrm{t}-\delta, \mathrm{t}]$ e que $\ell$ é contínua. Assim, dada a função:

$$
\mathrm{m}(\mathrm{s})=1+\mid \mathrm{f}(\mathrm{s}, \varphi(0), \varphi(\ell(\mathrm{s})) \mid
$$


definida em $(t-\delta, t]$, temos que $m=m$, em quase toda parte, sendo $m$ contínua (e limitada sobre seu domínio).

Logo, m é Lebesgue Integrável em ( $\mathrm{t}-\delta, \mathrm{t}$ ] e conforme [19] página 80 , a funçăo $m$ é também Lebesgue Integrável em $(t-\delta, t]$.

Por outro lado, $m$ é Lebesgue Integrável em $[t, t+\delta)$ pois é contínua e limitada neste intervalo.

Assim, m é Lebesgue Integrável no seu domínio. 


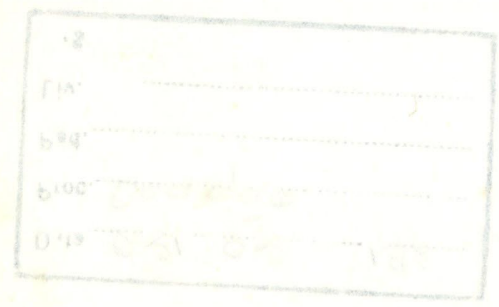

